\title{
The non-thermal Sunyaev-Zel'dovich effect in clusters of galaxies
}

\author{
S. Colafrancesco ${ }^{1}$, P. Marchegiani ${ }^{2}$, and E. Palladino ${ }^{2}$ \\ 1 INAF - Osservatorio Astronomico di Roma via Frascati 33, 00040 Monteporzio, Italy \\ 2 Dipartimento di Fisica, Università di Roma "La Sapienza”, Piazzale A. Moro, 2 Roma, Italy
}

Received 21 May 2002 / Accepted 13 August 2002

\begin{abstract}
In this paper we provide a general derivation of the non-thermal Sunyaev-Zel'dovich (SZ) effect in galaxy clusters which is exact in the Thomson limit to any approximation order in the optical depth $\tau$. The general approach we use also allows us to obtain an exact derivation of the thermal SZ effect in a self-consistent framework. Such a general derivation is obtained using the full relativistic formalism and overcoming the limitations of the Kompaneets and of the single scattering approximations. We compare our exact results with those obtained at different approximation orders in $\tau$ and we give estimates of the precision fit. We verified that the third order approximation yields a quite good description of the spectral distortion induced by the Comptonization of CMB photons in the cluster atmosphere. In our general derivation, we show that the spectral shape of the thermal and non-thermal SZ effect depends not only on the frequency but also on the cluster parameters, like the electron pressure and optical depth and from the energy spectrum of the electron population. We also show that the spatial distribution of the thermal and non-thermal SZ effect in clusters depends on a combination of the cluster parameters and on the spectral features of the effect. To have a consistent description of the SZ effect in clusters containing non-thermal phenomena, we also evaluate in a consistent way - for the first time - the total SZ effect produced by a combination of thermal and nonthermal electron population residing in the same environment, like is the case in radio-halo clusters. In this context, we show that the location of the zero of the total SZ effect increases non-linearly with increasing values of the pressure ratio between the non-thermal and thermal electron populations and its determination provides a unique way to determine the pressure of the relativistic particles residing in the cluster atmosphere. We discuss in detail both the spectral and the spatial features of the total (thermal plus non-thermal) SZ effect and we provide specific predictions for a well studied radio-halo cluster like A2163. Our general derivation allows also to discuss the overall SZ effect produced by a combination of different thermal populations residing in the cluster atmosphere. Such a general derivation of the SZ effect allows to consider also the CMB Comptonization induced by several electron populations. In this context, we discuss how the combined observations of the thermal and non-thermal SZ effect and of the other non-thermal emission features occurring in clusters (radio-halo, hard X-ray and EUV excesses) provide relevant constraints of the spectrum of the relativistic electron population and, in turn, on the presence and on the origin of non-thermal phenomena in galaxy clusters. We finally discuss how SZ experiments with high sensitivity and narrow-band spectral coverage, beyond the coming PLANCK satellite, can definitely probe the presence of a non-thermal SZ effect in galaxy clusters and disentangle this source of bias from the cosmologically relevant thermal SZ effect.
\end{abstract}

Key words. cosmology: theory - galaxies: clusters: general

\section{Introduction}

Compton scattering of the Cosmic Microwave Background (CMB) radiation by hot Intra Cluster (hereafter IC) electrons - the Sunyaev Zel'dovich effect (Zel'dovich \& Sunyaev 1969; Sunyaev \& Zel'dovich 1972, 1980) - is an important process whose spectral imprint on the CMB can be used as a powerful astrophysical and cosmological probe (see Birkinshaw 1999 for a review). Such a scattering produces a systematic shift of the CMB photons from the Rayleigh-Jeans (RJ) to the Wien side of the spectrum.

An approximate description of the scattering of an isotropic Planckian radiation field by a non-relativistic Maxwellian

Send offprint requests to: $\mathrm{S}$. Colafrancesco,

e-mail: cola@coma.mporzio.astro.it electron population can be obtained by means of the solution of the Kompaneets (1957) equation (see, e.g., Sunyaev \& Zel'dovich 1980). The resulting change in the spectral intensity, $\Delta I_{\mathrm{th}}$, due to the scattering of CMB photons by a thermal electron distribution can be written as

$\Delta I_{\mathrm{th}}=2 \frac{\left(k_{\mathrm{B}} T_{0}\right)^{3}}{(h c)^{2}} y_{\mathrm{th}} g(x)$,

where $x=h v / k_{\mathrm{B}} T_{0}$ is the a-dimensional frequency and the spectral shape of the effect is contained in the function

$g(x)=\frac{x^{4} \mathrm{e}^{x}}{\left(\mathrm{e}^{x}-1\right)^{2}}\left[x \frac{\mathrm{e}^{x}+1}{\mathrm{e}^{x}-1}-4\right]$

which is zero at the frequency $x_{0}=3.83$ (or $v=217 \mathrm{GHz}$ for a value of the CMB temperature $T_{0}=2.726 \mathrm{~K}$ ), negative 
at $x<x_{0}$ (in the RJ side) and positive at $x>x_{0}$ (in the Wien side). The Comptonization parameter, $y_{\text {th }}$, due to the thermal $\mathrm{SZ}$ effect is given by

$y_{\text {th }}=\frac{\sigma_{\mathrm{T}}}{m_{\mathrm{e}} c^{2}} \int \mathrm{d} \ell n_{\mathrm{e}} k_{\mathrm{B}} T_{\mathrm{e}}$,

where $n_{\mathrm{e}}$ and $T_{\mathrm{e}}$ are the electron density and temperature of the IC gas, respectively, $\sigma_{\mathrm{T}}$ is the Thomson cross section, valid in the limit $T_{\mathrm{e}} \gg T_{0}, k_{\mathrm{B}}$ is the Boltzmann constant and $m_{\mathrm{e}} c^{2}$ is the rest mass energy of the electron. The Comptonization parameter is proportional to the integral along the line of sight $\ell$ of the kinetic pressure, $P_{\mathrm{th}}=n_{\mathrm{e}} k_{\mathrm{B}} T_{\mathrm{e}}$, of the IC gas. Thus, the previous Eq. (3) can be written as

$y_{\mathrm{th}}=\frac{\sigma_{\mathrm{T}}}{m_{\mathrm{e}} c^{2}} \int \mathrm{d} \ell P_{\mathrm{th}}$

where the relevant dependence from the total kinetic pressure, $P_{\text {th }}$, of the clusters along the line of sight $\ell$ appears.

The previous description of the thermal SZ effect is obtained under the Kompaneets (1957) approximation and in the single scattering regime of the true photon redistribution function. As such, it only provides an approximation of the SZ effect in galaxy clusters for low temperatures $\left(T_{\mathrm{e}} \lesssim 3 \mathrm{keV}\right)$ and low optical depth $\left(\tau=\sigma_{\mathrm{T}} \int \mathrm{d} \ell n_{\mathrm{e}} \lesssim 10^{-3}\right)$.

However, recent X-ray observations have revealed the existence of many high-temperature clusters (David et al. 1993; Arnaud et al. 1994; Markevitch et al. 1994; Mushotzky \& Scharf 1997) with $k_{\mathrm{B}} T_{\mathrm{e}}$ up to $\sim 17 \mathrm{keV}$ (see, e.g., Tucker et al. 1998). Thus, the calculation of the thermal SZ effect from these hot clusters requires us to take into account the appropriate relativistic corrections (see Birkinshaw 1999 and references therein).

Analytical expressions for the SZ effect in the relativistic case have been considered by various authors (Stebbins 1997; Itoh et al. 1998; Challinor \& Lasenby 1998; see also Birkinshaw 1999 and references therein). Some of these calculations (out of the Monte Carlo simulations) are still approximate since they have been carried out in the following limits: i) single scattering of CMB photons against the IC gas electrons; ii) diffusion limit in which the use of the Kompaneets equation is justified. Itoh et al. (1998) obtained higher-order relativistic corrections to the thermal SZ effect in the form of a Fokker-Planck expansion (generalised Kompaneets equation) and by direct integration of the Boltzmann collision term. Such an integration has been carried out in the full relativistic regime. So, the results of Itoh et al. (1998) can be regarded as exact in the framework of the single scattering approximation. Analytical fitting formulae of such derivation can be found in Nozawa et al. (2000) and Itoh et al. (2002). Such a formalism seems to offer a detailed description of the thermal SZ effect for $k_{\mathrm{B}} T_{\mathrm{e}} \lesssim 15 \mathrm{keV}$, while Monte Carlo simulations describe more correctly the thermal SZ effect even for $k_{\mathrm{B}} T_{\mathrm{e}} \gtrsim 20 \mathrm{keV}$ (see, e.g., Challinor \& Lasenby 1998). Recently, Dolgov et al. (2001) proposed an approach based on an analytical reduction of the collision integral which is contained in the Boltzmannlike collision equation for the SZ effect produced by a thermal population of electrons. They found a numerical solution for the thermal SZ effect which is valid for generic values of $\tau$ and
$T_{\mathrm{e}}$. Sazonov \& Sunyaev (2000) presented a derivation of the monochromatic redistribution function in the mildly relativistic limit which considers also quantum effects and the use of the Klein-Nishina cross-section. However, they still consider only the single Compton scattering limit and the relativistic corrections up to some intermediate order due to low-energy photons and relativistic electrons. Such derivation of the monochromatic redistribution function in the limit $h v \ll k_{\mathrm{B}} T_{\mathrm{e}}$ reproduces the results of Fargion et al. (1997). More recently, Itoh et al. (2001) also presented a calculation of the thermal SZ effect which considers the contribution from multiple scattering in the relativistic limit. These last authors concluded that the multiple scattering contribution to the thermal SZ effect is negligible in galaxy clusters.

Another general assumption which is made in the calculation of the SZ effect is the use of a single population of thermal electrons which constitutes the hot (with temperature $T_{\mathrm{e}} \sim 10^{7}-10^{8} \mathrm{~K}$ ), optically thin (with density $n_{\mathrm{e}} \sim 10^{-3}$ $10^{-2} \mathrm{~cm}^{-3}$, and size $R \sim$ a few Mpc) Intra Cluster Medium (hereafter ICM). This assumption is based on the evidence that the IC gas is mainly constituted by thermal electrons (and protons) which are responsible for the X-ray emission observed in many clusters through thermal bremsstrahlung radiation (see Sarazin 1988 for a review).

Nonetheless, in addition to the thermal IC gas, many galaxy clusters contain a population of relativistic electrons which produce a diffuse radio emission (radio halos and/or relics) via synchrotron radiation in a magnetized ICM (see, e.g., Feretti 2000 for a recent observational review). The electrons which are responsible for the radio halo emission must have energies $E_{\mathrm{e}} \gtrsim$ a few $\mathrm{GeV}$ to radiate at frequencies $v \gtrsim 30 \mathrm{MHz}$ in order to reproduce the main properties of the observed radio halos (see, e.g., Blasi \& Colafrancesco 1999; Colafrancesco $\&$ Mele 2001, and references therein). A few nearby clusters also show the presence of an EUV/soft X-ray excess (Lieu et al. 1999; Kaastra et al. 1999 2002; Bowyer 2000) and of an hard X-ray excess (Fusco-Femiano et al. 1999-2000; Rephaeli et al. 1999; Kaastra et al. 1999; Henriksen 1999) over the thermal bremsstrahlung radiation. These emission excesses over the thermal X-ray emission may be produced either by Inverse Compton Scattering (hereafter ICS) of CMB photons off an additional population of relativistic electrons or by a combination of thermal (reproducing the EUV excess, Lieu et al. 2000) and suprathermal (reproducing the hard X-ray excess by nonthermal bremsstrahlung, Blasi et al. 2000; Dogiel 2000; Sarazin \& Kempner 2000) populations of distinct origins. However, since the inefficient non-thermal bremsstrahlung mechanism would require a large energy input and thus imply an excessive heating of the IC gas - which is not observed - a more complex electron population is required to fit both the radio halo and the EUV/hard X-ray spectra of galaxy clusters (Petrosian 2001). The high-energy part $(E \gtrsim 1 \mathrm{GeV})$ of such spectrum contains non-thermal electrons and produce synchrotron radio emission which can fit the observed radio-halo features: this is the best studied region of the non-thermal spectrum in galaxy clusters.

Indeed, in many of the clusters in which the SZ effect has been detected there is also evidence for radio halo sources (see Colafrancesco 2002). So it is of interest to assess whether the 
detected SZ effect is in fact mainly produced by the thermal electron population or there is a relevant contribution from other non-thermal electron populations. We will show specifically in this paper that each one of the electron populations which reside in the cluster atmosphere produces a distinct SZ effect with peculiar spectral and spatial features.

The description of the non-thermal SZ effect produced by a single electron population with a non-thermal spectrum has been attempted by various authors (McKinnon et al. 1991; Birkinshaw 1999; Ensslin \& Kaiser 2000; Blasi et al. 2000). Several limits to the non-thermal SZ effect are available in the literature (see, e.g., Birkinshaw 1999 for a review) from observations of galaxy clusters which contain powerful radio halo sources (such as A2163) or radio galaxies (such as A426), but only a few detailed analysis of the results (in terms of putting limits to the non-thermal SZ effect) have been possible so far. Only a single devoted search for the SZ effect expected from a relativistic population of electrons in the lobes of bright radio galaxies has been attempted to date (McKinnon et al. 1991). No signals were seen and a detailed spectral fit of the data to separate residual synchrotron and SZ effect signals was not done, so that the limits on the SZ effect do not strongly constrain the electron populations in the radio lobes. Also the problem of detecting the non-thermal SZ effect in radio-halo clusters is likely to be severe because of the associated synchrotron radio emission. In fact, at low radio frequencies, such a synchrotron emission could easily dominate over the small negative signal produced by the SZ effect. At higher frequencies there is in principle more chance to detect the non-thermal SZ effect, but even here there are likely to be difficulties in separating the $\mathrm{SZ}$ effect from the flat-spectrum component of the synchrotron emission (Birkinshaw 1999).

From the theoretical point of view, preliminary calculations (Birkinshaw 1999; Ensslin \& Kaiser 2000; Blasi et al. 2000) of the non-thermal SZ effect have been carried out in the diffusion approximation $(\tau \ll 1)$, in the limit of single scattering and for a single non-thermal population of electrons. Specifically, Ensslin \& Kaiser (2000) and Blasi et al. (2000) considered the SZ effect produced under the previous approximations by a supra-thermal tail of the Maxwellian electron distribution claimed to exist in the Coma cluster and concluded that the SZ effect, even though of small amplitude, could be measurable in the sub-mm region by the next coming PLANCK experiment. However, Petrosian (2001) showed that the suprathermal electron distribution faces with several crucial problems, the main being the large heating that such electrons would induce through Coulomb collisions in the ICM. The large energy input of the suprathermal distribution in the ICM of Coma would heat the IC gas up to unreasonably high temperatures, $k_{\mathrm{B}} T_{\mathrm{e}} \sim 10^{16} \mathrm{~K}$, which are not observed. In addition, Colafrancesco (2002) noticed that dust obscuration does not allow any detection of the SZ signal from Coma at frequencies $\gtrsim 600 \mathrm{GHz}$.

Matters are significantly more complicated if the full relativistic formalism is used. However, this is necessary, since many galaxy clusters show extended radio halos and the electrons which produce the diffuse synchrotron radio emission are certainly highly relativistic so that the use of the Kompaneets approximation is invalid. Moreover, the presence of thermal and non-thermal electrons in the same location of the ICM renders the single scattering approximation and the single population approach unreasonable, so that the treatment of multiple scattering among different electronic populations coexisting in the same cluster atmosphere is necessary to describe correctly the overall SZ effect.

Here we derive the spectral and spatial features of the SZ effect using an exact derivation of the spectral distortion induced by a combination of a thermal and a non-thermal population of electrons which are present at the same time in the ICM. Lately, we also consider the case of the combination of several non-thermal and thermal populations. The plan of the paper is the following: in Sect. 2 we will provide a general derivation of the SZ effect in an exact formalism within the framework of our approach, i.e., considering the fully relativistic approach outlined in Birkinshaw (1999) and the effect induced by multiple scattering. We work here in the Thomson limit $h v \ll m_{\mathrm{e}} c^{2}$. Within such framework, we derive the exact SZ effect for a single electron population both in the thermal and non-thermal cases. In Sect. 3 we derive the SZ effect produced by a single population of non-thermal electrons providing both exact and approximate (up to any order in $\tau$ ) expressions for the nonthermal SZ effect. In Sect. 4 we derive the total SZ effect produced by a combination of two populations: a distribution of thermal electrons - like that responsible for the X-ray emission of galaxy clusters - and a non-thermal electron distribution like the one responsible for the radio halo emission which is present in many galaxy clusters. Our general approach allows to derive the expression for the SZ effect produced by the combination of any electronic population. So, in Sect. 5 we consider also the SZ effect generated by the combination of two different thermal populations. In Sect. 6 we discuss the spatial features associated to the presence of a non-thermal SZ effect superposed to the thermal SZ effect. In Sect. 7 we derive limits on the presence and amplitude of the non-thermal SZ effect discussing specifically the cases of a few clusters in which there is evidence for the presence of non-thermal, high-energy electrons. We show how the possible detection of a non-thermal SZ effect can set relevant constraints on the relative electron population. We summarize our results and discuss our conclusions in the final Sect. 8. We use $H_{0}=50 \mathrm{~km} \mathrm{~s}^{-1} \mathrm{Mpc}^{-1}$ and $\Omega_{0}=1$ throughout the paper unless otherwise specified.

\section{The SZ effect for galaxy clusters: A generalized approach}

In this section we derive a generalized expression for the SZ effect which is valid in the Thomson limit for a generic electron population in the relativistic limit and includes also the effects of multiple scattering. First we consider an expansion in series for the distorted spectrum $I(x)$ in terms of the optical depth, $\tau$, of the electron population. Then, we consider an exact derivation of the spectral distortion using the Fourier Transform method already outlined in Birkinshaw (1999).

An electron with momentum $p=\beta \gamma$, with $\beta=v / c$ and $\gamma=E_{\mathrm{e}} / m_{\mathrm{e}} c^{2}$ increases the frequency $v$ of a scattered CMB photon on average by the factor $t \equiv v^{\prime} / v=\frac{4}{3} \gamma^{2}-\frac{1}{3}$, where $v^{\prime}$ 
and $v$ are the photon frequencies after and before the scattering, respectively. Thus, in the Compton scattering against relativistic electrons $(\gamma \gg 1)$ a CMB photon is effectively removed from the $\mathrm{CMB}$ spectrum and is found at much higher frequencies.

We work here in the Thomson limit, (in the electron's rest frame $\gamma h v \ll m_{\mathrm{e}} c^{2}$ ), which is valid for the interesting range of frequencies at which SZ observations are feasible.

The redistribution function of the CMB photons scattered once by the IC electrons writes in the relativistic limit as,

$P_{1}(s)=\int_{0}^{\infty} \mathrm{d} p f_{\mathrm{e}}(p) P_{\mathrm{s}}(s ; p)$

where $f_{\mathrm{e}}(p)$ is the electron momentum distribution and $P_{\mathrm{s}}(s ; p)$ is the redistribution function for a mono-energetic electron distribution, with $s \equiv \ln (t)$. An analytical expression for the redistribution function of CMB photons which suffer a single scattering, $P_{\mathrm{s}}(s ; p)$, has been given by Ensslin \& Kaiser (2000). The expression for such a redistribution function has been also given analytically by Fargion et al. (1997) and by Sazonov \& Sunyaev (2000). Once the function $P_{1}(s)$ is known, it is possible to evaluate the probability that a frequency change $s$ is produced by a number $n$ of repeated, multiple scattering. This is given by the repeated convolution

$$
\begin{aligned}
& P_{n}(s) \\
& \quad=\int_{-\infty}^{+\infty} \mathrm{d} s_{1} \ldots \mathrm{d} s_{n-1} P_{1}\left(s_{1}\right) \ldots P_{1}\left(s_{n-1}\right) P_{1}\left(s-s_{1}-\ldots-s_{n-1}\right) \\
& \equiv \underbrace{P_{1}(s) \otimes \ldots \otimes P_{1}(s)}_{n \text { times }}
\end{aligned}
$$

(see Birkinshaw 1999), where the symbol $\otimes$ indicates each convolution product. As a result, the location of the maximum of the function $P_{n}(s)$ moves towards higher values of $s$ for higher values of $n$ and the distribution $P_{n}(s)$ widens a-symmetrically towards high values of $s$ giving thus higher probabilities to have large frequency shifts. The resulting total redistribution function $P(s)$ can be written as the sum of all the functions $P_{n}(s)$, each one weighted by the probability that a CMB photon can suffer $n$ scatterings, which is assumed to be Poissonian with expected value $\tau$ :

$$
\begin{aligned}
P(s) & =\sum_{n=0}^{+\infty} \frac{\mathrm{e}^{-\tau} \tau^{n}}{n !} P_{n}(s)=\mathrm{e}^{-\tau}\left[P_{0}(s)+\tau P_{1}(s)+\frac{1}{2} \tau^{2} P_{2}(s)+\ldots\right] \\
& =\mathrm{e}^{-\tau}\left[\delta(s)+\tau P_{1}(s)+\frac{1}{2} \tau^{2} P_{1}(s) \otimes P_{1}(s)+\ldots\right]
\end{aligned}
$$

The spectrum of the Comptonized radiation is then given by

$I(x)=\int_{-\infty}^{+\infty} \mathrm{d} s I_{0}\left(x \mathrm{e}^{-s}\right) P(s)$,

where

$I_{0}(x)=2 \frac{\left(k_{\mathrm{B}} T_{0}\right)^{3}}{(h c)^{2}} \frac{x^{3}}{\mathrm{e}^{x}-1}$

is the incident CMB spectrum in terms of the a-dimensional frequency $x$.

In the following we will derive the expression for the distorted spectrum using first an expansion in series of $\tau$ and then the exact formulas obtained with the Fourier Transform (FT) method.

\subsection{High order $\tau$-expansion}

In the calculation of the SZ effect in galaxy clusters it is usual to use the expression of $P(s)$ which is derived in the single scattering approximation and in the diffusion limit, $\tau \ll 1$. In these limits, the distorted spectrum writes, in our formalism, as:

$I(x)=J_{0}(x)+\tau\left[J_{1}(x)-J_{0}(x)\right]$,

where

$$
\begin{aligned}
J_{0}(x) & =\int_{-\infty}^{+\infty} I_{0}\left(x \mathrm{e}^{-s}\right) P_{0}(s) \mathrm{d} s=\int_{-\infty}^{+\infty} I_{0}\left(x \mathrm{e}^{-s}\right) \delta(s) \mathrm{d} s \\
& =I_{0}(x) \\
J_{1}(x) & =\int_{-\infty}^{+\infty} I_{0}\left(x \mathrm{e}^{-s}\right) P_{1}(s) \mathrm{d} s .
\end{aligned}
$$

To evaluate the SZ distorted spectrum $I(x)$ up to higher order in $\tau$, we make use of the general expression of the series expansion of the function $P(s)$

$P(s)=\sum_{n=0}^{+\infty} a_{n}(s) \tau^{n}$

which can be written, using Eq. (7), as

$P(s)=\sum_{k=0}^{+\infty} \frac{(-\tau)^{k}}{k !} \sum_{k^{\prime}=0}^{+\infty} \frac{\tau^{k^{\prime}}}{k^{\prime} !} P_{k^{\prime}}(s)$

The general $n$th order term is obtained by selecting the terms in the double summation which contain the optical depth $\tau$ up to the $n$th power. These terms are obtained for $k^{\prime}=n-k$, and provide the following expression for the series expansion coefficients:

$a_{n}(s)=\sum_{k=0}^{n} \frac{(-1)^{k}}{k !(n-k) !} P_{n-k}(s)=\frac{1}{n !} \sum_{k=0}^{n}\left(\begin{array}{l}n \\ k\end{array}\right)(-1)^{k} P_{n-k}(s)$.

Inserting the coefficients $a_{n}(s)$ given in Eq. (15) in Eq. (13) and this last one in Eq. (8), the resulting spectral distortion due to the SZ effect can be written as:

$I(x)=\sum_{n=0}^{+\infty} b_{n}(x) \tau^{n}$

where the coefficients $b_{n}(x)$ are given by

$b_{n}(x)=\frac{1}{n !} \sum_{k=0}^{n}\left(\begin{array}{l}n \\ k\end{array}\right)(-1)^{k} J_{n-k}(x)$,

and

$J_{n-k}(x)=\int_{-\infty}^{+\infty} I_{0}\left(x \mathrm{e}^{-s}\right) P_{n-k}(s) \mathrm{d} s$.

In this general approach, we can write the corrections to the distorted spectrum $I(x)$ up to any order $n$ in the quantity $\tau$. 


\subsection{Exact derivation of the $S Z$ effect}

The redistribution function $P(s)$ can also be obtained in an exact form considering all the terms of the series expansion in Eq. (14). In fact, since the Fourier transform (hereafter FT) of a convolution product of two functions is equal to the product of the Fourier transforms of the two functions, the FT of $P(s)$ writes as

$$
\begin{aligned}
\tilde{P}(k) & =\mathrm{e}^{-\tau}\left[1+\tau \tilde{P}_{1}(k)+\frac{1}{2} \tau^{2} \tilde{P}_{1}^{2}(k)+\ldots\right]=\mathrm{e}^{-\tau} \mathrm{e}^{\tau \tilde{P}_{1}(k)} \\
& =\mathrm{e}^{-\tau\left[1-\tilde{P}_{1}(k)\right]}
\end{aligned}
$$

where

$\tilde{P}_{1}(k)=\int_{-\infty}^{+\infty} P_{1}(s) \mathrm{e}^{-i k s} \mathrm{~d} s$

(Taylor \& Wright 1989). The exact form of the Comptonized spectrum $I(x)$ is then given by Eq. (8) in terms of the exact redistribution function

$P(s)=\frac{1}{2 \pi} \int_{-\infty}^{+\infty} \tilde{P}(k) \mathrm{e}^{i k s} \mathrm{~d} k$

which is obtained as the anti Fourier transform of $\tilde{P}(k)$ given in Eq. (19). To compare the exact calculations of the SZ spectral distortion with those obtained in the non-relativistic limit (see Eqs. (1)-(2) as for the thermal case), it is useful to write the distorted spectrum in the form

$\Delta I(x)=2 \frac{\left(k_{\mathrm{B}} T_{0}\right)^{3}}{(h c)^{2}} y \tilde{g}(x)$,

where $\Delta I(x) \equiv I(x)-I_{0}(x)$. The spectral shape of the SZ effect is contained in the function

$\tilde{g}(x)=\left(\frac{\Delta I}{I_{0}}\right) \frac{1}{y} \frac{x^{3}}{\mathrm{e}^{x}-1}=\frac{\Delta i(x)}{y}$

where $\Delta i \equiv \Delta I \frac{(h c)^{2}}{2\left(k_{\mathrm{B}} T_{0}\right)^{3}}$. The Comptonization parameter $y$ is defined, in our general approach, in terms of the pressure $P$ of the considered electron population:

$y=\frac{\sigma_{\mathrm{T}}}{m_{\mathrm{e}} c^{2}} \int P \mathrm{~d} \ell$.

\subsection{The case of a single thermal electron population}

For a thermal electron population in the non-relativistic limit with momentum distribution $f_{\mathrm{e}, \mathrm{th}} \propto p^{2} \exp \left(-\eta \sqrt{1+p^{2}}\right)$ with $\eta=m_{\mathrm{e}} c^{2} / k_{\mathrm{B}} T_{\mathrm{e}}$, one writes the pressure as

$P_{\text {th }}=n_{\mathrm{e}} k_{\mathrm{B}} T_{\mathrm{e}}$

and it is easy from Eq. (24) to re-obtain the Compton parameter in Eq. (3) as

$y_{\text {th }}=\frac{\sigma_{\mathrm{T}}}{m_{\mathrm{e}} c^{2}} \int n_{\mathrm{e}} k_{\mathrm{B}} T_{\mathrm{e}} \mathrm{d} \ell=\tau \frac{k_{\mathrm{B}} T_{\mathrm{e}}}{m_{\mathrm{e}} c^{2}}$

(we consider here, for simplicity, an isothermal cluster). The relativistically correct expression of the function $\tilde{g}(x)$ for a thermal population of electrons writes, at first order in $\tau$, as:

$\tilde{g}(x)=\frac{\Delta i}{y_{\mathrm{th}}}=\frac{\tau\left[j_{1}-j_{0}\right]}{\tau \frac{k_{\mathrm{B}} T_{\mathrm{e}}}{m_{\mathrm{e}} c^{2}}}=\frac{m_{\mathrm{e}} c^{2}}{k_{\mathrm{B}} T_{\mathrm{e}}}\left[j_{1}-j_{0}\right]$, where $j_{i} \equiv J_{i} \frac{(h c)^{2}}{2\left(k_{\mathrm{B}} T_{0}\right)^{3}}$ and the functions $J_{i}$ are given in Eq. (18). In the same line, it is possible to write the expression of $\tilde{g}(x)$ up to higher orders in $\tau$. For example, limiting the series expansion in Eq. (14) at third order in $\tau$, we found:

$$
\begin{aligned}
\tilde{g}(x)= & \frac{m_{\mathrm{e}} c^{2}}{k_{\mathrm{B}} T_{\mathrm{e}}}\left[\left(j_{1}-j_{0}\right)+\frac{1}{2} \tau\left(j_{2}-2 j_{1}+j_{0}\right)\right. \\
& \left.+\frac{1}{6} \tau^{2}\left(j_{3}-3 j_{2}+3 j_{1}-j_{0}\right)\right] .
\end{aligned}
$$

Notice that while the expression derived for $\tilde{g}(x)$ at first order approximation in $\tau$ is independent of $\tau$, the expression for $\tilde{g}(x)$ at higher order approximation in $\tau$ depends directly on $\tau$. This is even more the case for the exact expression of $\tilde{g}(x)$ given in the following. In fact, using the exact form of the function $P(s)$ given in Eq. (22), it is possible to write the exact form of the function $\tilde{g}(x)$ as:

$\tilde{g}(x)=\frac{m_{\mathrm{e}} c^{2}}{k_{\mathrm{B}} T_{\mathrm{e}}}\left\{\frac{1}{\tau}\left[\int_{-\infty}^{+\infty} i_{0}\left(x \mathrm{e}^{-s}\right) P(s) \mathrm{d} s-i_{0}(x)\right]\right\}$.

The expression of $\tilde{g}(x)$ approximated at first order in $\tau$ as given by Eq. (27) is the one to compare directly with the expression of $g(x)$ obtained from the Kompaneets (1957) equation, since both are evaluated under the assumption of single scattering suffered by a CMB photon against the IC electrons. Figure 1 shows how the function $\tilde{g}(x)$ tends to $g(x)$ for lower and lower IC gas temperatures $T_{\mathrm{e}}$. This confirms that the distorted spectrum obtained from the Kompaneets equation is the non-relativistic limit of the exact spectrum. Notice that the function $\tilde{g}(x)$ given in Eq. (29) is the spectral shape of the SZ effect obtained in the exact calculation while the function $g(x)$ refers to the 1 st order approximated case of a single, thermal, non-relativistic population of electrons.

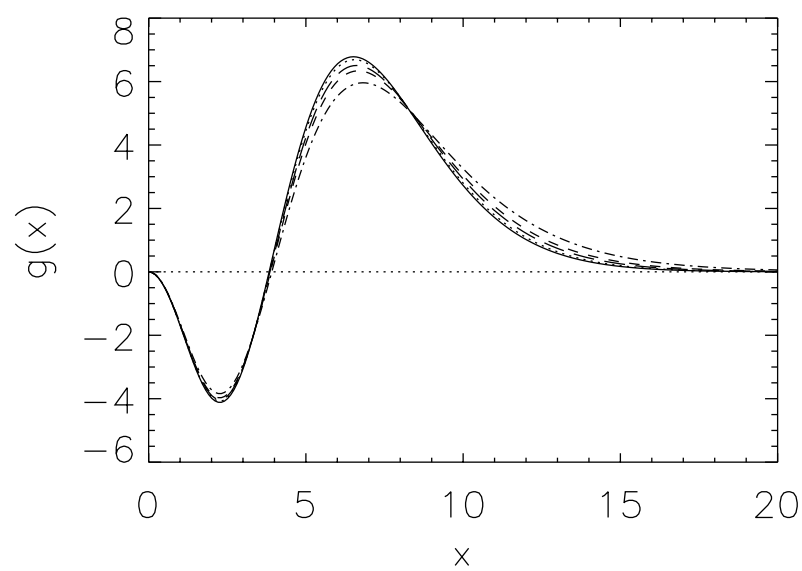

Fig. 1. The function $g(x)$ (solid line) is compared with the function $\tilde{g}(x)$ for thermal electron populations with $k_{\mathrm{B}} T_{\mathrm{e}}=10$ (dot-dashed), 5 (dashes), 3 (long dashes) and 1 (dotted) keV, respectively.

It is worth to notice that while in the non-relativistic case it is possible to separate the spectral dependence of the effect (which is contained in the function $g(x)$ ) from the dependence on the cluster parameters (which are contained in Compton parameter $y_{\text {th }}$, see Eqs. (1)-(3)), this is no longer valid in the relativistic case in which the function $J_{1}$ depends itself also on the 
Table 1. The fractional error $\varepsilon=|[g(x)-\tilde{g}(x)] / \tilde{g}(x)|$ computed for thermal electron populations with $k_{\mathrm{B}} T_{\mathrm{e}}=10,5,3,2,1 \mathrm{keV}$ is shown for different values of the frequency $x$. The quantity $\varepsilon$ is given in units of $10^{-2}$.

\begin{tabular}{lccccc}
\hline \hline & $k_{\mathrm{B}} T_{\mathrm{e}}=10 \mathrm{keV}$ & $k_{\mathrm{B}} T_{\mathrm{e}}=5 \mathrm{keV}$ & $k_{\mathrm{B}} T_{\mathrm{e}}=3 \mathrm{keV}$ & $k_{\mathrm{B}} T_{\mathrm{e}}=2 \mathrm{keV}$ & $k_{\mathrm{B}} T_{\mathrm{e}}=1 \mathrm{keV}$ \\
\hline$x=2.3$ & 7.20 & 3.68 & 2.26 & 1.57 & 0.93 \\
$x=6.5$ & 14.81 & 7.28 & 4.33 & 2.87 & 1.40 \\
$x=15$ & 64.84 & 45.99 & 32.67 & 23.87 & 13.15 \\
\hline
\end{tabular}

cluster parameters. Specifically, for a thermal electron distribution, $J_{1}$ depends non-linearly from the electron temperature $T_{\mathrm{e}}$ through the function $P_{1}(s)$. This means that, even at first order in $\tau$, the spectral shape $\tilde{g}(x)$ of the SZ effect depends on the cluster parameters, and mainly from the electron pressure $P_{\text {th }}$.

To evaluate the errors done by using the non-relativistic expression $g(x)$ instead of the relativistic, correct function $\tilde{g}(x)$ given in Eq. (29) we calculate the fractional error $\varepsilon=$ $|[g(x)-\tilde{g}(x)] / \tilde{g}(x)|$ for thermal populations with $k_{\mathrm{B}} T_{\mathrm{e}}=10,5$, 3,2 and $1 \mathrm{keV}$ and for three representative frequencies (see Table 1). The fractional errors in Table 1 tend to decrease systematically at each frequency with decreasing temperature. Note, however, that the error found in the high frequency region, $x=15$, is much higher than the errors found at lower frequencies and produces uncertainty levels of $\gtrsim 50 \%$ for $T_{\mathrm{e}} \gtrsim 5 \mathrm{keV}$. This indicates that the high frequency region of the SZ effect is more affected by the relativistic corrections and by multiple scattering effects. In Table 2 we show the fractional error $\varepsilon$ done when considering the exact calculation of the thermal SZ effect and those at first, second and third order approximations in $\tau$ for two values of the optical depth, $\tau=10^{-2}$ and $\tau=10^{-3}$, as reported in the table caption. Even for the highest cluster temperatures here considered, $k_{\mathrm{B}} T_{\mathrm{e}} \sim 20 \mathrm{keV}$, the difference between the exact and approximated calculations is $\lesssim 0.25 \%$ at the minimum of the SZ effect $(x \sim 2.3)$ and is $\lesssim 2.23 \%$ in the high-frequency tail $(x \sim 15)$, the two frequency ranges where the largest deviations are expected and could be measurable, in principle. For high-temperature clusters, the third-order approximated calculations of the SZ effect ensures a precision $\lesssim 2 \%$ at any interesting frequency.

Using the general, exact relativistic approach discussed in this section, we evaluate the frequency location of the zero of the thermal SZ effect, $x_{0}$, for different cluster temperatures in the range $2-20 \mathrm{keV}$. The frequency location of $x_{0}$ depends on the IC gas temperature (or more generally on the IC gas pressure) and in Fig. 2 we compare the temperature dependence of $x_{0}$ evaluated at first order in $\tau$ (which actually does not depend on $\tau$ ) with that evaluated in the exact approach for values $\tau=10^{-3}$ and $\tau=10^{-2}$. The location of the null of the SZ effect increases by $\sim 4.4 \%$ for $k_{\mathrm{B}} T_{\mathrm{e}}$ up to $\sim 20 \mathrm{keV}$. Assuming a quadratic fit $x_{0} \approx a+b \theta_{\mathrm{e}}+c \theta_{\mathrm{e}}^{2}$, where $\theta_{\mathrm{e}}=k_{\mathrm{B}} T_{\mathrm{e}} / m_{\mathrm{e}} c^{2}$, we calculate the coefficients $a, b$ and $c$ which fit the temperature dependence of $x_{0}$. These are given in Table 3. In particular the coefficients of the last row in Table 3 can be compared with those given by Itoh et al. (1998). At first order in $k_{\mathrm{B}} T_{\mathrm{e}}$, and for IC gas temperatures up to $50 \mathrm{keV}$, these last authors found $x_{0} \approx 3.830+4.471 \theta_{\mathrm{e}}-3.268 \theta_{\mathrm{e}}^{2}$. The coefficients given in the first two rows of Table 3 can be also compared with those found by Dolgov et al. (2000), who gave

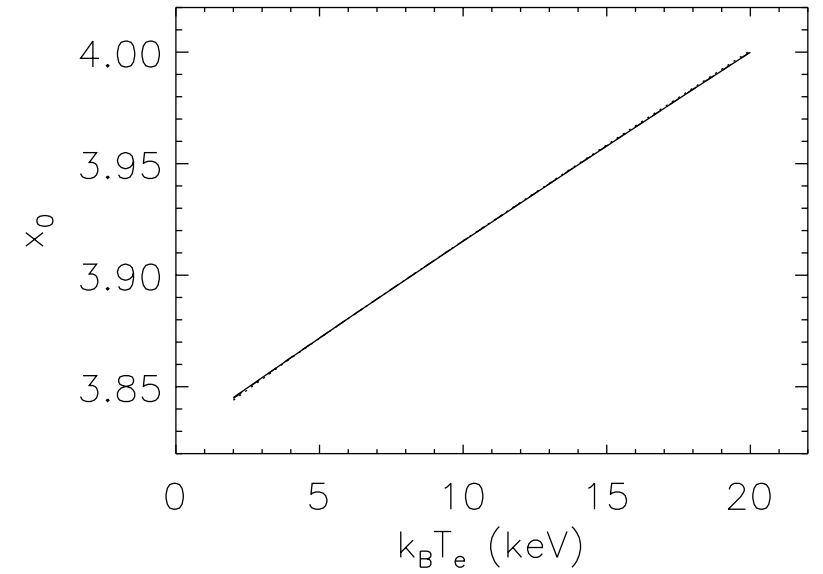

Fig. 2. The location of $x_{0}$ for a thermal electron population is shown as a function of $k_{\mathrm{B}} T_{\mathrm{e}}$. The exact calculation for $\tau=10^{-2}$ (solid line) and for $\tau=10^{-3}$ (dashed line) are shown together with the approximated calculation to first order in $\tau$ (dotted line).

Table 2. The fractional error $\varepsilon$ done considering the first, second and third order approximation in $\tau$ compared with the exact calculation of the thermal SZ effect is reported for three interesting values of the frequency $x$. The assumed values of $k_{\mathrm{B}} T_{\mathrm{e}}$ and $\tau$ are shown in the first column of the table. The values of $\varepsilon$ are given in units of $10^{-2}$.

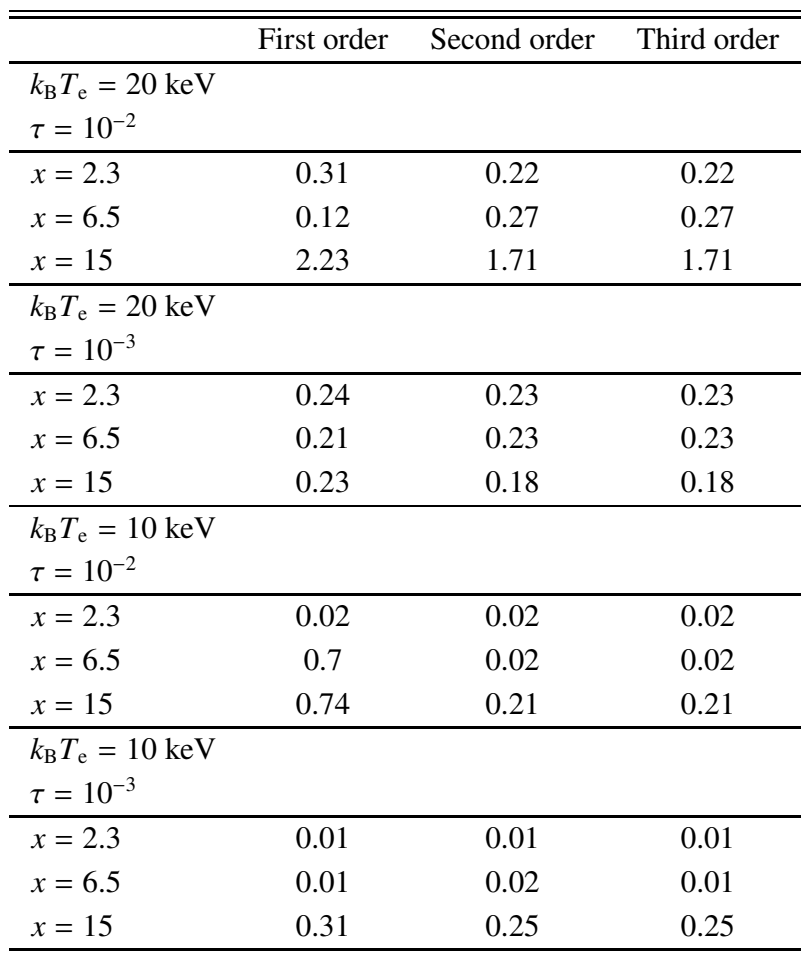


Table 3. The coefficients of the quadratic fit of the behaviour $x_{0, \mathrm{th}}$ as a function of $\theta_{\mathrm{e}}=k_{\mathrm{B}} T_{\mathrm{e}} / m_{\mathrm{e}} c^{2}$ (see text for details).

\begin{tabular}{lccc}
\hline \hline & $a$ & $b$ & $c$ \\
\hline Exact $\tau=0.001$ & 3.8271 & 4.6059 & -4.8221 \\
Exact $\tau=0.01$ & 3.8262 & 4.6704 & -5.4895 \\
First order & 3.8294 & 4.4304 & -1.7805 \\
\hline
\end{tabular}

the expression of $x_{0}$ evaluated in an exact way for a temperature range $0 \leq k_{\mathrm{B}} T_{\mathrm{e}} \leq 50 \mathrm{keV}$ and for $0 \leq \tau \leq 0.05$ : $x_{0}=\alpha\left(T_{\mathrm{e}}\right)+\tau \beta\left(T_{\mathrm{e}}\right)$ with $\alpha\left(T_{\mathrm{e}}\right)=3.830\left(1+1.162 \theta_{\mathrm{e}}-0.8144 \theta_{\mathrm{e}}^{2}\right)$ and $\beta\left(T_{\mathrm{e}}\right)=3.021 \theta_{\mathrm{e}}-8.672 \theta_{\mathrm{e}}^{2}$.

For $\tau=0.01$, the expression for $x_{0}$ derived by Dolgov et al. (2000) writes as

$x_{0}=3.830+4.481 \theta_{\mathrm{e}}-3.206 \theta_{\mathrm{e}}^{2}$,

while for $\tau=0.001$ one gets

$x_{0}=3.830+4.453 \theta_{\mathrm{e}}-3.128 \theta_{\mathrm{e}}^{2}$.

The remaining differences with our coefficients given in Table 3 are due to the different $T_{\mathrm{e}}$ and $\tau$ ranges used for the quadratic fit.

\section{The SZ effect generated from a non-thermal electron population}

Using the same general analytical approach derived in Sect. 2 above we derive here the exact spectral features of the SZ effect produced by a single non-thermal population of electrons. Such an exact derivation has been not done so far. For such a population we consider here two different phenomenological spectra: i) a single power-law energy spectrum, $n_{\text {rel }}=n_{0} E^{-\alpha}$, like that which is able to fit the radio-halo spectra of many clusters, and ii) a double power-law spectrum which is able to fit both the radio halo spectrum and the EUV and hard X-ray excess spectra observed in some nearby clusters (see Petrosian 2001). Our formalism is, nonetheless, so general that it can be applied to any electron distribution so far considered to fit both the observed radio-halo spectra and those of the EUV/Hard X-ray excesses (see, e.g., Sarazin 1999; Blasi \& Colafrancesco 1999; Colafrancesco \& Mele 2001; Petrosian 2001).

The single power-law electron population is described by the momentum spectrum

$f_{\mathrm{e}, \text { rel }}\left(p ; p_{1}, p_{2}, \alpha\right)=A\left(p_{1}, p_{2}, \alpha\right) p^{-\alpha} ; \quad p_{1} \leq p \leq p_{2}$

where the normalization term $A\left(p_{1}, p_{2}, \alpha\right)$ is given by:

$A\left(p_{1}, p_{2}, \alpha\right)=\frac{(\alpha-1)}{p_{1}^{1-\alpha}-p_{2}^{1-\alpha}}$

with $\alpha \approx 2.5$. This is the simplest electron distribution which is consistent with the spectra of the radio halos observed in many galaxy clusters (see, e.g., Feretti 2001 for a recent review). In the calculation of the non-thermal SZ effect we consider the minimum momentum, $p_{1}$, of the electron distribution as a free parameter since it is not constrained by the available observations, while the specific value of $p_{2} \gg 1$ is irrelevant for powerlaw indices $\alpha>2$ which are required by the radio halo spectra observed in galaxy clusters.

The distribution in Eq. (32) can be however affected by a crucial problem: if the electron spectrum is extended at energies below $20 \mathrm{MeV}$ (i.e., at $p \lesssim 40$ ) with the same spectral slope $\alpha \approx 2.5$ of the high energy tail, the heating rate of the IC gas produced by Coulomb collisions of the relativistic electrons becomes larger than the bremsstrahlung cooling rate of the IC gas (see, e.g., Petrosian 2001). This would produce unreasonably and unacceptably large heating of the IC gas.

For this reason, we consider also a double power-law electron spectrum which has a flatter slope below a critical value $p_{\mathrm{cr}}$ :

$$
\begin{aligned}
& f_{\mathrm{e}, \mathrm{rel}}\left(p ; p_{1}, p_{2}, p_{\mathrm{cr}}, \alpha_{1}, \alpha_{2}\right) \\
& \quad=K\left(p_{1}, p_{2}, p_{\mathrm{cr}}, \alpha_{1}, \alpha_{2}\right) \begin{cases}p^{-\alpha_{1}} & p_{1}<p<p_{\mathrm{cr}} \\
p_{\mathrm{cr}}^{-\alpha_{1}+\alpha_{2}} p^{-\alpha_{2}} & p_{\mathrm{cr}}<p<p_{2}\end{cases}
\end{aligned}
$$

with a normalization factor given by:

$$
\begin{aligned}
K\left(p_{1}, p_{2}, p_{\mathrm{cr}}, \alpha_{1}, \alpha_{2}\right)= & {\left[\frac{p_{1}^{1-\alpha_{1}}-p_{\mathrm{cr}}^{1-\alpha_{1}}}{\alpha_{1}-1}\right.} \\
& \left.+p_{\mathrm{cr}}^{-\alpha_{1}+\alpha_{2}} \frac{p_{\mathrm{cr}}^{1-\alpha_{2}}-p_{2}^{1-\alpha_{2}}}{\alpha_{2}-1}\right]^{-1} .
\end{aligned}
$$

If $p_{\text {cr }} \sim 400$, the electron distribution of Eq. (34) with $\alpha_{1} \sim 0.5$ at $p \lesssim p_{\text {cr }}$ can be extended down to very low energies without violating any constraint set by the IC gas heating. Hence, we assume here the following parameter values: $\alpha_{1}=0.5, \alpha_{2}=$ $2.5, p_{\text {cr }}=400$ e $p_{2} \rightarrow \infty$. We again consider $p_{1}$ as a free parameter.

A crucial quantity in the calculation of the non-thermal SZ effect is given by the number density of relativistic electrons, $n_{\mathrm{e}, \text { rel }}$. The quantity $n_{\mathrm{e}, \text { rel }}$ in galaxy clusters can be estimated from the radio halo spectrum intensity, but this estimate depends on the assumed value of the IC magnetic field and on the model for the evolution of the radio-halo spectrum (see, e.g. Sarazin 1999 for time-dependent models and Blasi \& Colafrancesco 1999; Colafrancesco \& Mele 2001 for stationary models). For the sake of illustration, the radio halo flux $J_{v}$ for a power-law spectrum is, in fact, given by $J_{v} \propto n_{\mathrm{e}, \mathrm{rel}} B^{\alpha_{\mathrm{r}}-1} v^{-\alpha_{\mathrm{r}}}$, where $\alpha_{\mathrm{r}}$ is the radio halo spectral slope. Because of the large intrinsic uncertainties existing in the density of the relativistic electrons which produce the cluster non-thermal phenomena, a value $n_{\mathrm{e}, \text { rel }}=10^{-6} \mathrm{~cm}^{-3}$ for $p_{1}=100$ has been assumed here.

Our results on the amplitude of the non-thermal SZ effect can be easily rescaled to different values of $n_{\mathrm{e}, \text { rel }}$ : in fact, decreasing (increasing) $n_{\mathrm{e}, \text { rel }}$ will produce smaller (larger) amplitudes of the SZ effect, as can be seen from Eqs. (22), (24), (28), (29).

The density $n_{\mathrm{e}, \text { rel }}$ increases for decreasing values of $p_{1}$. In fact, multiplying the electron distribution in Eq. (32) by the quantity $n_{\mathrm{e}, \text { rel }}\left(p_{1}\right)$ one obtains

$N_{\mathrm{e}}\left(p ; p_{1}\right) \equiv n_{\mathrm{e}, \mathrm{rel}}\left(p_{1}\right) A\left(p_{1}\right) p^{-\alpha}$ 
where the function $A\left(p_{1}\right)$ is given by Eq. (33). Thus, the electron density scales as

$n_{\mathrm{e}, \mathrm{el}}\left(p_{1}\right)=n_{\mathrm{e}, \mathrm{rel}}\left(\tilde{p_{1}}\right) \frac{A\left(\tilde{p_{1}}\right)}{A\left(p_{1}\right)}$,

where we normalized the electron density at a fixed value of $\tilde{p_{1}}=100$.

The value of $p_{1}$ sets the value of the electron density as well as the value of the other relevant quantities which depend on it, namely the optical depth $\tau_{\text {rel }}$ and the pressure $P_{\text {rel }}$ of the non-thermal population. In particular, the pressure $P_{\text {rel }}$ for the case of an electron distribution in Eq. (32), is given by

$$
\begin{aligned}
P_{\text {rel }} & =n_{\mathrm{e}} \int_{0}^{\infty} \mathrm{d} p f_{\mathrm{e}}(p) \frac{1}{3} p v(p) m_{\mathrm{e}} c \\
& =\frac{n_{\mathrm{e}} m_{\mathrm{e}} c^{2}(\alpha-1)}{6\left[p^{1-\alpha}\right]_{p_{2}}^{p_{1}}}\left[B \frac{1}{1+p^{2}}\left(\frac{\alpha-2}{2}, \frac{3-\alpha}{2}\right)\right]_{p_{2}}^{p_{1}}
\end{aligned}
$$

(see, e.g., Ensslin \& Kaiser 2000), where $B_{x}$ is the incomplete Beta function

$B_{x}(a, b)=\int_{0}^{x} t^{a-1}(1-t)^{b-1} \mathrm{~d} t$

(see, e.g., Abramowitz \& Stegun 1965). The optical depth of the non-thermal electron population is given by

$\tau_{\text {rel }}\left(p_{1}\right)=2 \times 10^{-6} \frac{A\left(\tilde{p_{1}}\right)}{A\left(p_{1}\right)}\left[\frac{n_{\mathrm{e}, \mathrm{rel}}\left(\tilde{p_{1}}=100\right)}{10^{-6} \mathrm{~cm}^{-3}}\right]\left[\frac{\ell}{1 \mathrm{Mpc}}\right]$.

For an electron population as in Eq. (34) we obtain analogous results. The electron density with the same normalization as before is given by

$n_{\mathrm{e}, \mathrm{rel}}\left(p_{1}\right)=n_{\mathrm{e}, \mathrm{rel}}\left(\tilde{p_{1}}\right) \frac{K\left(\tilde{p_{1}}\right)}{K\left(p_{1}\right)}$.

The optical depth is again given by Eq. (40), where we substitute the ratio $K\left(\tilde{p_{1}}\right) / K\left(p_{1}\right)$ to $A\left(\tilde{p_{1}}\right) / A\left(p_{1}\right)$. The electron pressure of this last population has instead a more complicated expression given by:

$$
\begin{aligned}
P_{\text {rel }}= & \frac{K\left(p_{1}\right) n_{\mathrm{e}}\left(p_{1}\right) m_{\mathrm{e}} c^{2}}{6}\left\{\left[B \frac{1}{1+p^{2}}\left(\frac{\alpha_{1}-2}{2}, \frac{3-\alpha_{1}}{2}\right)\right]_{p_{\mathrm{cr}}}^{p_{1}}\right. \\
& \left.+\left[B \frac{1}{1+p^{2}}\left(\frac{\alpha_{2}-2}{2}, \frac{3-\alpha_{2}}{2}\right)\right]_{p_{2}}^{p_{\mathrm{cr}}} p_{\mathrm{cr}}^{-\alpha_{1}+\alpha_{2}}\right\} .
\end{aligned}
$$

To evaluate the SZ effect induced by a single non-thermal electron population we use the relativistically correct formalism described in Sect. 2 which also takes into account the effects of multiple scattering. Again, we are working here in the Thomson limit. The general expression for the distorted spectrum $I(x)$ is given again by Eq. (8). The only change in our general formulation consists in inserting the non-thermal distribution $f_{\mathrm{e}, \text { non-th }}$ in the function $P_{1}(s)$. The functions $P_{n}(s)$, which determine the probabilities to have a frequency shift $s$ due to $n$ scattering are still given by the convolution of $n$ distributions $P_{1}(s)$ (see Eq. (6)). Figure 3 shows the function $P_{1}(s)$ for different values of $p_{1}$ in the range $0.5-1000$. For low values of $p_{1}$, the function $P_{1}(s)$ is centered around $s=0$ and does not produce, hence, large frequency changes. For higher values of

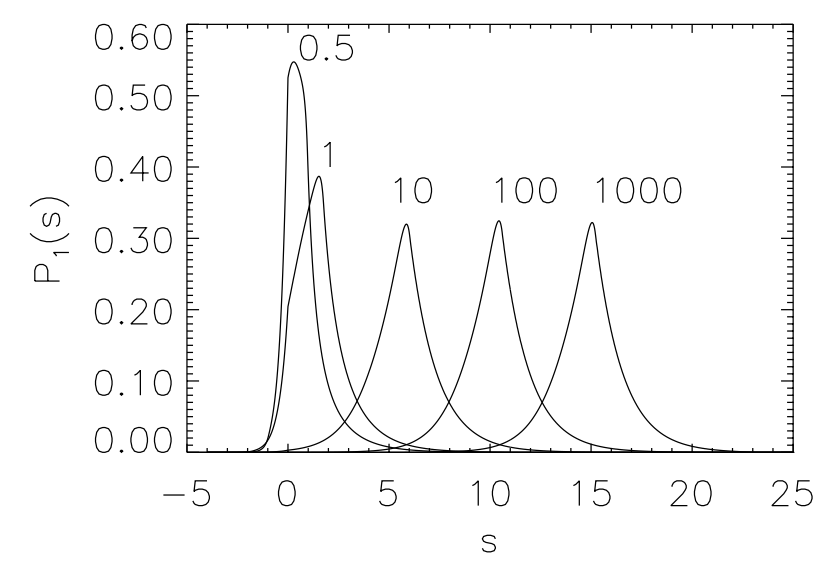

Fig. 3. The distribution $P_{1}(s)$ for a non-thermal population as in Eq. (32) with $\alpha=2.5, p_{2} \rightarrow \infty$ and $p_{1}=0.5,1,10,100,1000$, as indicated.

$p_{1}$ (i.e., increasing values of the minimum energy of the electrons) the function $P_{1}(s)$ becomes wider and it is centered at higher and higher values of $s$. For $p_{1}=1000$ the redistribution function $P_{1}(s)$ is centered around $s=15$, which corresponds to a frequency change $v^{\prime} / v=\mathrm{e}^{s} \sim 3 \times 10^{6}$. Thus, the SZ effect caused by electronic populations with very high values of $p_{1}$ moves the photon frequencies from the CMB region up to very different regions causing a depletion of scattered $\mathrm{CMB}$ photons at sub-mm wavelengths where the SZ effect is usually studied. We report in Table 4 the values of the pressure and of the density of a single power-law population as a function of $p_{1}$.

Table 4. Values of the pressure and of the density of a single powerlaw population as in Eq. (32) with $\alpha=2.5, p_{2} \rightarrow \infty$ and $n_{\text {e,rel }}\left(\tilde{p}_{1}=\right.$ 100) $=1 \times 10^{-6} \mathrm{~cm}^{-3}$ for different values of $p_{1}$.

\begin{tabular}{ccc}
\hline \hline$p_{1}$ & $P_{\text {rel }}\left(\mathrm{keV} \mathrm{cm}^{-3}\right)$ & $n_{\mathrm{e}, \mathrm{rel}}\left(\mathrm{cm}^{-3}\right)$ \\
\hline 0.5 & 0.59 & $2.83 \times 10^{-3}$ \\
1 & 0.47 & $1 \times 10^{-3}$ \\
10 & 0.16 & $3.16 \times 10^{-5}$ \\
100 & $5.11 \times 10^{-2}$ & $1 \times 10^{-6}$ \\
1000 & $1.61 \times 10^{-2}$ & $3.16 \times 10^{-8}$ \\
\hline
\end{tabular}

To emphasize the changes in the photon redistribution function produced by a non-thermal electron distribution, we show in Fig. 4 the functions $P_{1}(s), P_{2}(s)$ and $P_{3}(s)$ for a single and double power-law electron distributions. It is evident that these functions are wider and more a-symmetrically skewed towards large and positive values of $s$ with respect to the same distributions evaluated in the case of a single thermal electron population. Once the exact redistribution function $P(s)$ is known, it is possible to evaluate the exact distorted spectrum $I(x)$ through Eq. (8).

It is also possible to evaluate the spectral distortion using Eq. (16) which gives the series expansion up to the $n$th order in $\tau$ and to compare the exact and approximated shapes for $I(x)$. We show in Fig. 5 the first three terms $b_{1}, b_{2}, b_{3}$ of the series expansion in Eq. (16) as a function of the a-dimensional frequency $x$. The approximated expression for $I(x)$ up to third 

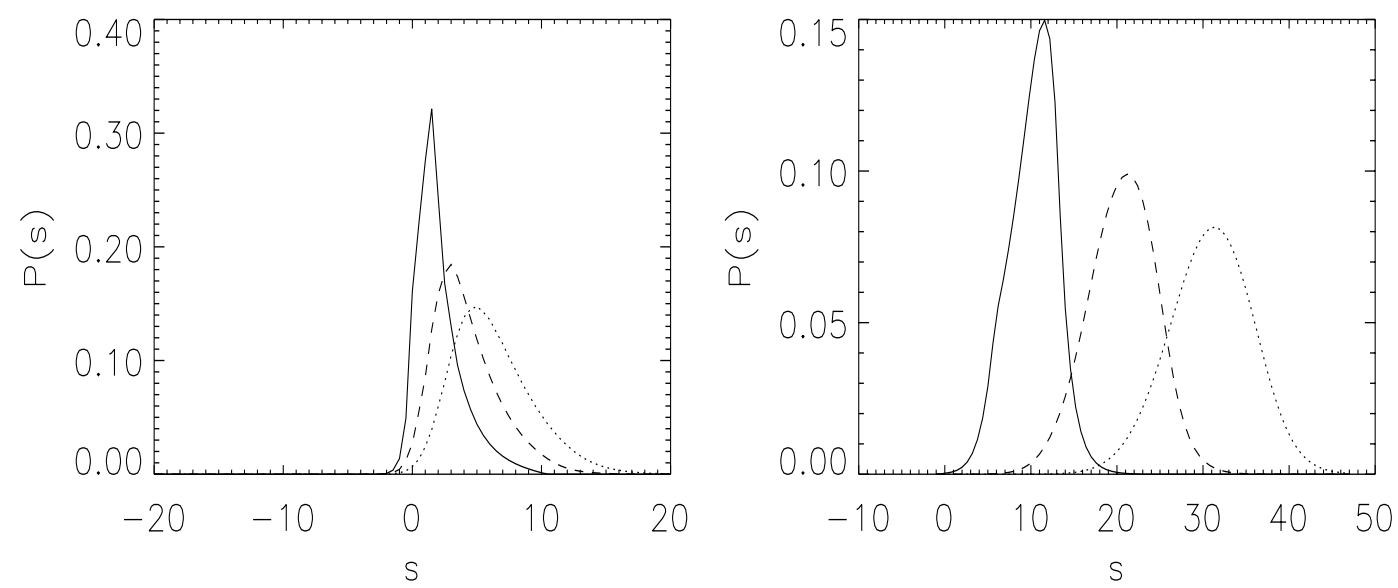

Fig. 4. The distributions $P_{1}(s)$ (solid line), $P_{2}(s)$ (dashed) and $P_{3}(s)$ (dotted) evaluated for non-thermal electron populations with a single power-law spectrum given in Eq. (32) with $\alpha=2, p_{1}=1$ and $p_{2}=100$ (left panel) and with a double power-law spectrum given in Eq. (34) (right panel). Note that these distributions are wider and more skewed towards larger values of $s$ than the corresponding distributions evaluated in the case of a thermal electron population.

order in $\tau$ is an excellent fit of its exact behaviour at frequencies around the minimum of the effect $(x \approx 2.3)$ and it shows a difference $\sim 3 \%$ at $x \approx 6.5$ and a difference $\approx 0.1 \%$ at high frequencies, $x \approx 15$ (see Table 5). The precision of the approximation depends also on the value of $\tau$ and we verified that the precision of the second and third order approximations does not increase for values $\tau \lesssim 10^{-3}$ at any frequency between $x \approx 2$ and $x \approx 15$.

Table 5. Fractional difference (in units of $10^{-2}$ ) between the exact spectral distortion and that evaluated at first, second and third order in $\tau$. We report here the cases for values of the optical depth $\tau=0.01$ and $\tau=0.001$.

\begin{tabular}{lccc}
\hline \hline & First order & Second order & Third order \\
\hline$\tau=0.01$ & & & \\
\hline$x=2.3$ & 0.82 & 0.44 & 0.44 \\
$x=6.5$ & 1.42 & 2.90 & 2.90 \\
$x=15$ & 0.83 & 0.12 & 0.12 \\
\hline$\tau=0.001$ & & & \\
\hline$x=2.3$ & 0.49 & 0.45 & 0.45 \\
$x=6.5$ & 2.73 & 2.88 & 2.88 \\
$x=15$ & 0.19 & 0.12 & 0.12 \\
\hline
\end{tabular}

\subsection{The function $\tilde{g}(x)$ for a single non-thermal population}

Also for a non-thermal electron population it is possible to write the spectral distortion in the general form of Eq. (22) as:

$\Delta I_{\text {non-th }}(x)=2 \frac{\left(k_{\mathrm{B}} T_{0}\right)^{3}}{(h c)^{2}} y_{\text {non-th }} \tilde{g}(x)$,

where the Comptonization parameter is given by the general expression

$y_{\text {non-th }}=\frac{\sigma_{\mathrm{T}}}{m_{\mathrm{e}} c^{2}} \int P_{\mathrm{rel}} \mathrm{d} \ell$,

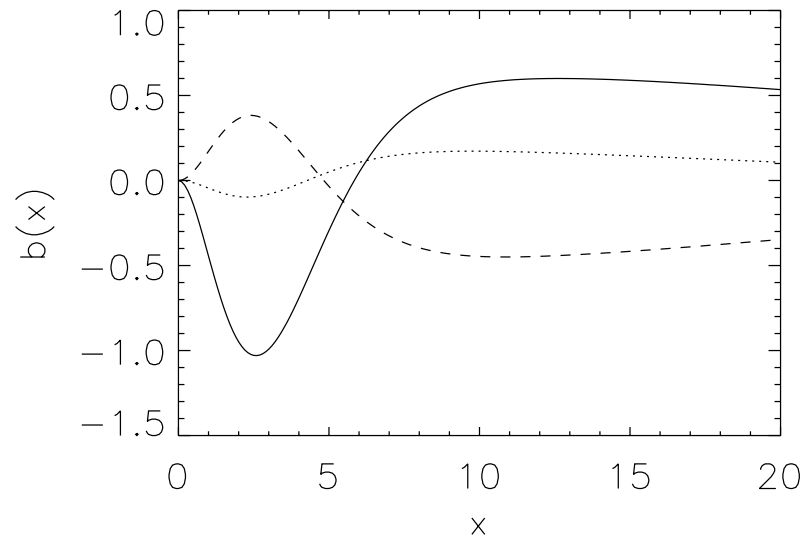

Fig. 5. The functions $b_{1}(x)$ (solid), $b_{2}(x)$ (dashed) and $b_{3}(x)$ (dotted) evaluated for a non-thermal electron population with a single powerlaw spectrum as in Eq. (32).

in terms of the electronic pressure $P_{\text {rel }}$ which, for a single and double power-law populations, is given by Eq. (38) and by Eq. (42), respectively.

At first order in $\tau$ we can write the spectral function $\tilde{g}(x)$ of the non-thermal SZ effect as

$\tilde{g}(x)=\frac{\Delta i}{y}=\frac{\tau\left[j_{1}-j_{0}\right]}{\frac{\sigma_{\mathrm{T}}}{m_{\mathrm{e}} c^{2}} \int P \mathrm{~d} \ell} \equiv \frac{m_{\mathrm{e}} c^{2}}{\left\langle k_{\mathrm{B}} T_{\mathrm{e}}\right\rangle}\left[j_{1}-j_{0}\right]$

where we defined the quantity

$\left\langle k_{\mathrm{B}} T_{\mathrm{e}}\right\rangle \equiv \frac{\sigma_{\mathrm{T}}}{\tau} \int P \mathrm{~d} \ell=\frac{\int P \mathrm{~d} \ell}{\int n_{\mathrm{e}} \mathrm{d} \ell}=\int_{0}^{\infty} \mathrm{d} p f_{\mathrm{e}}(p) \frac{1}{3} p v(p) m_{\mathrm{e}} c$

which is the analogous of the average temperature for a thermal population. In fact, comparing Eq. (46) with Eq. (25), $\left\langle k_{\mathrm{B}} T_{\mathrm{e}}\right\rangle=k_{\mathrm{B}} T_{\mathrm{e}}$ obtains. For a non-thermal population with a single power-law distribution one gets

$\left\langle k_{\mathrm{B}} T_{\mathrm{e}}\right\rangle=\frac{m_{\mathrm{e}} c^{2}(\alpha-1)}{6\left[p^{1-\alpha}\right]_{p_{2}}^{p_{1}}}\left[B_{\frac{1}{1+p^{2}}}\left(\frac{\alpha-2}{2}, \frac{3-\alpha}{2}\right)\right]_{p_{2}}^{p_{1}}$, 
while in the case of a double power-law distribution we find instead

$$
\begin{aligned}
\left\langle k_{\mathrm{B}} T_{\mathrm{e}}\right\rangle= & \frac{K\left(p_{1}\right) m_{\mathrm{e}} c^{2}}{6}\left\{\left[B \frac{1}{1+p^{2}}\left(\frac{\alpha_{1}-2}{2}, \frac{3-\alpha_{1}}{2}\right)\right]_{p_{\text {cr }}}^{p_{1}}\right. \\
& \left.+\left[B \frac{1}{1+p^{2}}\left(\frac{\alpha_{2}-2}{2}, \frac{3-\alpha_{2}}{2}\right)\right]_{p_{2}}^{p_{\text {cr }}} p_{\mathrm{cr}}^{-\alpha_{1}+\alpha_{2}}\right\} .
\end{aligned}
$$

It is possible to write the Comptonization parameter $y_{\text {non-th }}$ as a function of the quantity $\left\langle k_{\mathrm{B}} T_{\mathrm{e}}\right\rangle$ and of the optical depth $\tau$ :

$$
\begin{aligned}
y_{\text {non-th }} & =\frac{\sigma_{\mathrm{T}}}{m_{\mathrm{e}} c^{2}} \int P_{\text {rel }} \mathrm{d} \ell=\sigma_{\mathrm{T}} \frac{\left\langle k_{\mathrm{B}} T_{\mathrm{e}}\right\rangle}{m_{\mathrm{e}} c^{2}} \int n_{\mathrm{e}, \text { rel }} \mathrm{d} \ell \\
& =\frac{\left\langle k_{\mathrm{B}} T_{\mathrm{e}}\right\rangle}{m_{\mathrm{e}} c^{2}} \tau
\end{aligned}
$$

(note that here $\tau=\tau_{\text {rel }}$ holds).

We can also write the $n$th order approximations of the distorted spectrum using Eq. (16). For example, at third order in $\tau$, the following explicit expression founds:

$$
\begin{aligned}
\tilde{g}(x)= & \frac{m_{\mathrm{e}} c^{2}}{\left\langle k_{\mathrm{B}} T_{\mathrm{e}}\right\rangle}\left[\left(j_{1}-j_{0}\right)+\frac{1}{2} \tau\left(j_{2}-2 j_{1}+j_{0}\right)\right. \\
& \left.+\frac{1}{6} \tau^{2}\left(j_{3}-3 j_{2}+3 j_{1}-j_{0}\right)\right] .
\end{aligned}
$$

Finally, the exact form of the function $\tilde{g}(x)$ for a non-thermal electron population writes as:

$\tilde{g}(x)=\frac{m_{\mathrm{e}} c^{2}}{\left\langle k_{\mathrm{B}} T_{\mathrm{e}}\right\rangle}\left\{\frac{1}{\tau}\left[\int_{-\infty}^{+\infty} i_{0}\left(x \mathrm{e}^{-s}\right) P(s) \mathrm{d} s-i_{0}(x)\right]\right\}$.

We show in Fig. 6 a comparison between the function $g(x)$ obtained under the Kompaneets approximation (see Eq. (2)) and the function $\tilde{g}(x)$, approximated to first order in $\tau$, and evaluated for a single power-law population. A major difference between the two functions is the different position of the zero of the SZ effect which is moved to higher frequencies in the case of the non-thermal population with respect to the case of the thermal one. In Fig. 7 we compare the function $\tilde{g}(x)$ for a non-thermal population evaluated at first order in $\tau$ (which is actually independent from the value of $\tau$ ) with the exact function for values $\tau=1, \tau=0.1$ and $\tau=0.01$. We notice that the first order approximation is a good approximation of the exact case for values $\tau<10^{-3}$. Figure 8 shows the function $\tilde{g}(x)$ (evaluated at first order in $\tau$ ) for a single power-law population and considering different values of $p_{1}$. For increasing values of $p_{1}$ the maximum of the function $\tilde{g}(x)$ moves towards higher and higher frequencies. As a consequence, also the zero of the nonthermal SZ effect moves to higher values of $x$ as shown also in Fig. 9. The location of $x_{0}$ changes rapidly with increasing values of $p_{1}$ and reaches a value $x_{0} \approx 22$ for $p_{1}=2 \times 10^{3}$. At such values of $p_{1}$ the pressure $P_{\text {rel }}$ takes values $\approx 0.02 \mathrm{keV} \mathrm{cm}^{-3}$, as shown in Fig. 9. An important point to notice is that the electron density of the non-thermal population decreases for increasing values of $p_{1}$ as was already shown in Table 4 . For this reason the pressure $P_{\text {rel }}$ and the resulting spectral distortion decreases in amplitude for increasing values of $p_{1}$. This result is shown more specifically in Fig. 10 where we plot the quantity

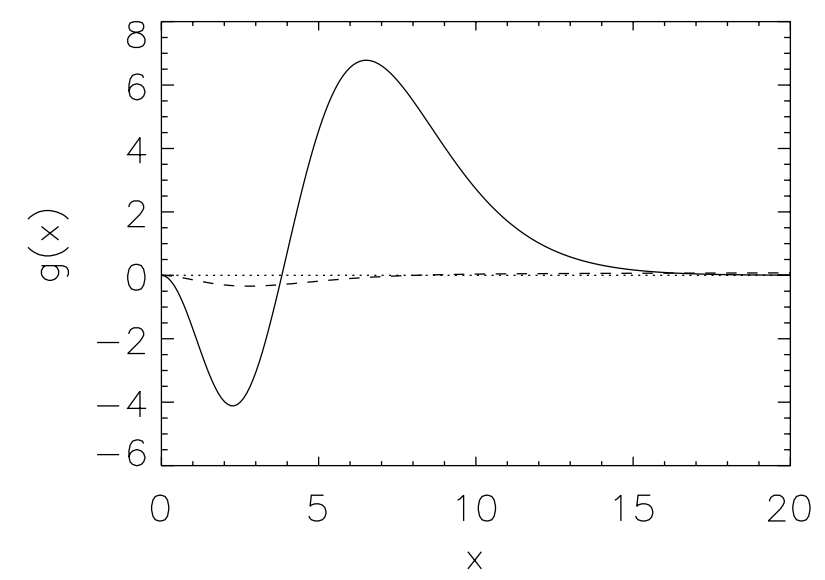

Fig. 6. The spectral function $g(x)$ for a thermal population in the Kompaneets limit (solid line) is compared with the function $\tilde{g}(x)$ approximated at first order in $\tau$ for a non-thermal population with single power-law spectrum with parameters $p_{1}=4, p_{2}=10^{10}$ and $\alpha=2.5$ (dashed line), evaluated from Eq. (45). The dotted line represents the zero reference value.

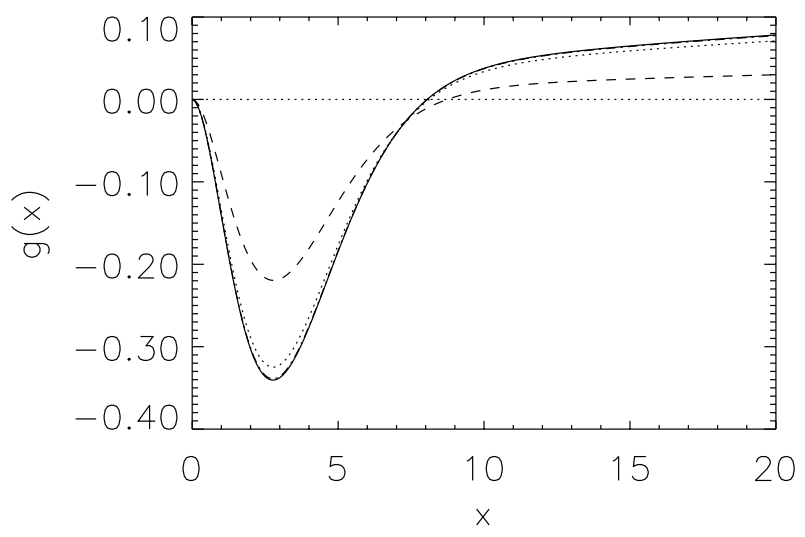

Fig. 7. The function $\tilde{g}(x)$ for a non-thermal population with single power-law spectrum with parameters $p_{1}=4, p_{2}=10^{10}$ and $\alpha=2.5$. We show the first order approximation in $\tau$ (solid line) and the exact case for $\tau=1$ (dashed line), $\tau=0.1$ (dotted line) and $\tau=0.01$ (dotdashed line). Note that the first order approximation is a reasonable approximation of the exact case only for small values of $\tau$.

$\Delta i(x)=\Delta I(x)\left[(h c)^{2} / 2\left(k_{\mathrm{B}} T_{0}\right)^{3}\right]$ for values of $p_{1}$ in the range $0.5-10^{3}$.

For an electron population with a double power-law spectrum as in Eq. (34) the values of the density and pressure are also reported in Table 6 as a function of $p_{1}$ (note that the case $p_{1}=1000$ is the same of the single power-law spectrum). The values of $P_{\text {rel }}$ and $n_{\mathrm{e} \text {,rel }}$ for low values of $p_{1}$ are now smaller than those of the single power-law case (see Table4), thus avoiding the problem of an excessive heating of the cluster IC gas for low values of $p_{1}$.

In Fig. 11 we show the function $P_{1}(s)$ evaluated for an electron population with double power-law spectrum (see Eq. (34)) and its dependence on the lower momentum cutoff $p_{1}$. The behaviour of $P_{1}(s)$ in this case is quite different with respect to the single power-law case (see Fig. 3) and depends mainly on the flatness of the electron spectrum below the momentum cutoff $p_{\text {cr. }}$. We notice that $i$ ) even for low values of $p_{1} \sim 0.5$ small 

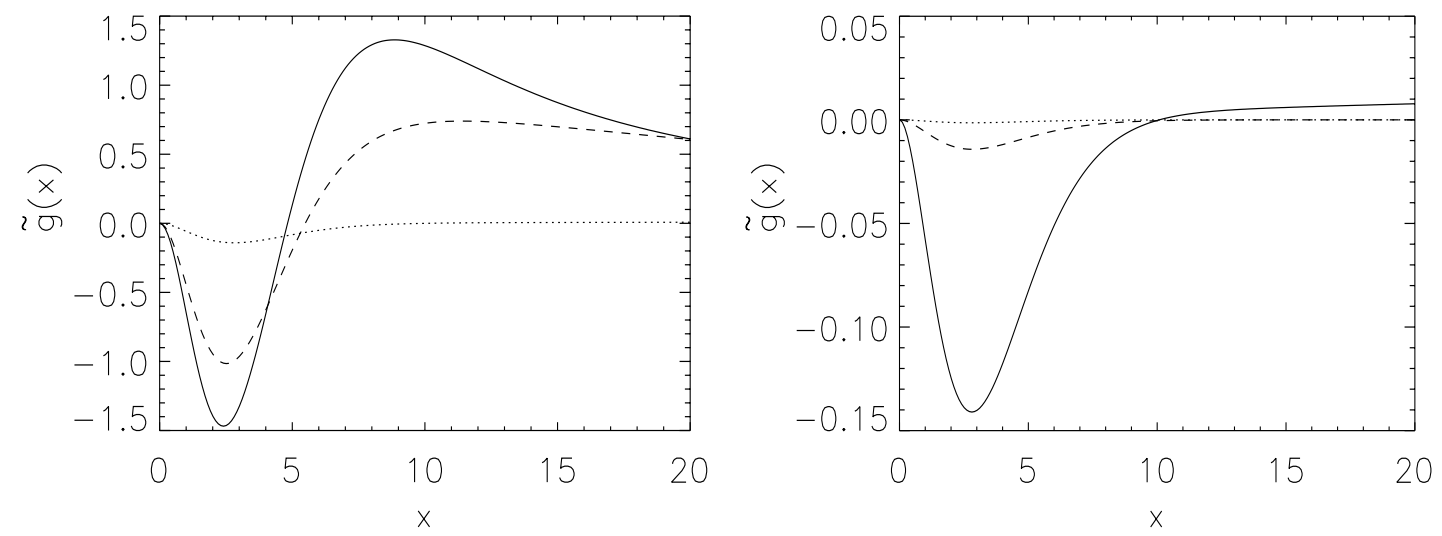

Fig. 8. In the left panel we show the function $\tilde{g}(x)$ (see Eq. (45)) for a non-thermal population as in Eq. (32) with $p_{1}=0.5$ (solid line), 1 (dashed) and 10 (dotted). The right panel shows the same function for $p_{1}=10$ (solid line), 100 (dashed) and 1000 (dotted).
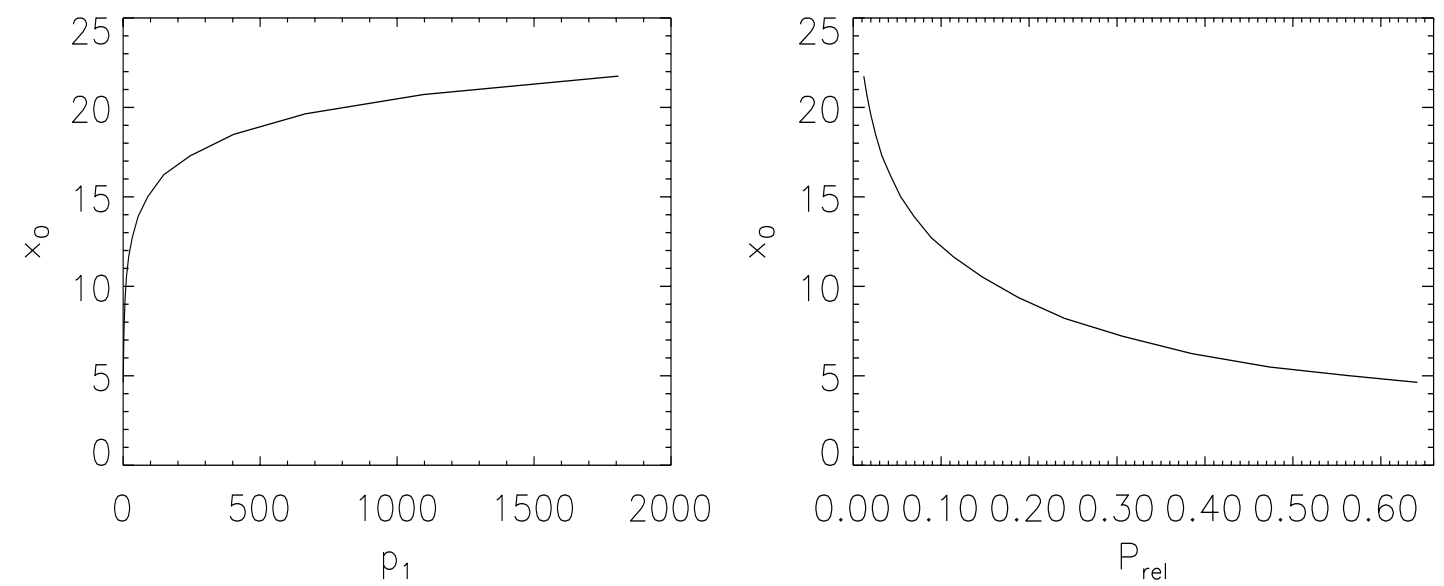

Fig. 9. The behaviour of the zero of the SZ effect for a non-thermal population given in Eq. (32) as a function of $p_{1}$ (left panel) and of the pressure $P_{\text {rel }}$ (right panel) expressed in $\mathrm{keV} \mathrm{cm}^{-3}$. Note that the pressure $P_{\text {rel }}$ decreases for increasing values of $p_{1}$, as discussed in Sect. 3

Table 6. Values of the pressure and of the density of a double powerlaw population as in Eq. (34) with $\alpha_{1}=0.5, \alpha_{2}=2.5, p_{\text {cr }}=400$, $p_{2} \rightarrow \infty$ and $n_{\mathrm{e}, \text { rel }}\left(\tilde{p}_{1}=100\right)=1 \times 10^{-6} \mathrm{~cm}^{-3}$ for different values of $p_{1}$.

\begin{tabular}{ccc}
\hline \hline$p_{1}$ & $P_{\text {rel }}\left(\mathrm{keV} \mathrm{cm}^{-3}\right)$ & $n_{\text {e,rel }}\left(\mathrm{cm}^{-3}\right)$ \\
\hline 0.5 & $1.26 \times 10^{-3}$ & $1.56 \times 10^{-6}$ \\
1 & $1.26 \times 10^{-3}$ & $1.54 \times 10^{-6}$ \\
10 & $1.23 \times 10^{-3}$ & $1.41 \times 10^{-6}$ \\
100 & $9.88 \times 10^{-4}$ & $1 \times 10^{-6}$ \\
\hline
\end{tabular}

frequency changes are unlikely, as shown by the strongly asymmetric shape of $P_{1}(s)$; ii) there are small differences in the function $P_{1}(s)$ for different values of $p_{1} \lesssim p_{\text {cr }}$ because the electron density in this range is lower than in the single power-law case and its variation is mild. We also show in Fig. 12 the function $\tilde{g}(x)$ evaluated at first order in $\tau$ for a double powerlaw electron population as in Eq. (34). In this case, there is no well defined maximum of the SZ effect since low frequency changes are unlikely. Moreover, the function $\tilde{g}(x)$ is less sensitive to different values of the lower momentum cutoff $p_{1}$ for $p_{1}<p_{\mathrm{cr}}$ because of the flatness of the electron spectrum in this region. This fact is also confirmed by the variation of the zero location of the non-thermal SZ effect (see Fig. 13). The

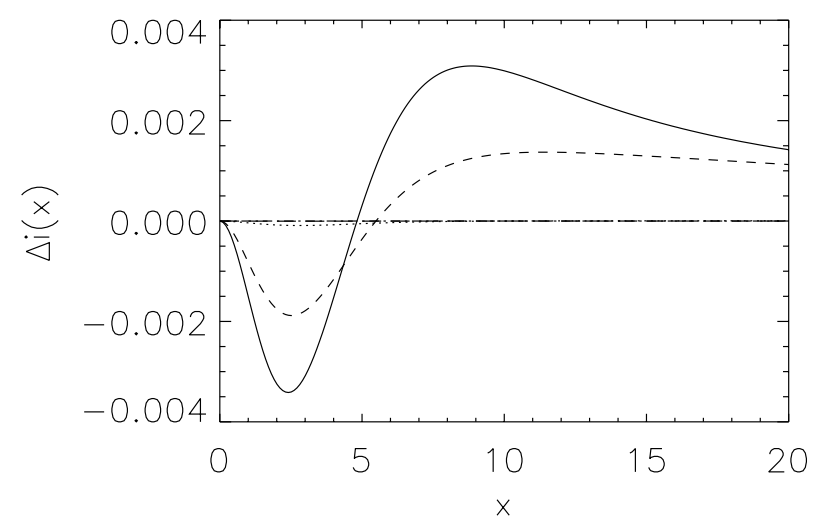

Fig. 10. The spectral distortion $\Delta i(x)$ in units of $2\left(k_{\mathrm{B}} T_{0}\right)^{3} /(h c)^{2}$ for a non-thermal population as in Eq. (32) evaluated for $p_{1}=0.5$ (solid line), 1 (dashes), 10 (dotted), 100 (long dashes) and 1000 (dot-dashes).

location of $x_{0}$ changes now less rapidly than in the case of a single power-law distribution (see Fig. 11) due to the different shape of $\tilde{g}(x)$, and hence of $P(s)$, caused by the lower density of the low-energy electrons with respect to the case of the single power-law spectrum. The zero of the non-thermal SZ effect produced by a single non-thermal population is also shifted to frequencies $x_{0} \gtrsim 10$ (much higher than the value $x_{0, \text { th }}=3.83$ ) 


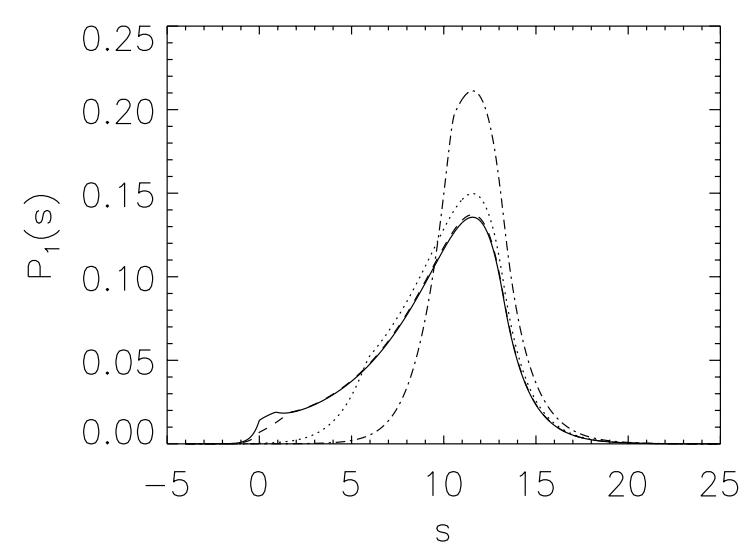

Fig. 11. The function $P_{1}(s)$ (see Eq. (5)) is shown for non-thermal populations given in Eq. (34) with $p_{1}=0.5$ (solid), 1 (dashes), 10 (dotted), 100 (dot-dashes).

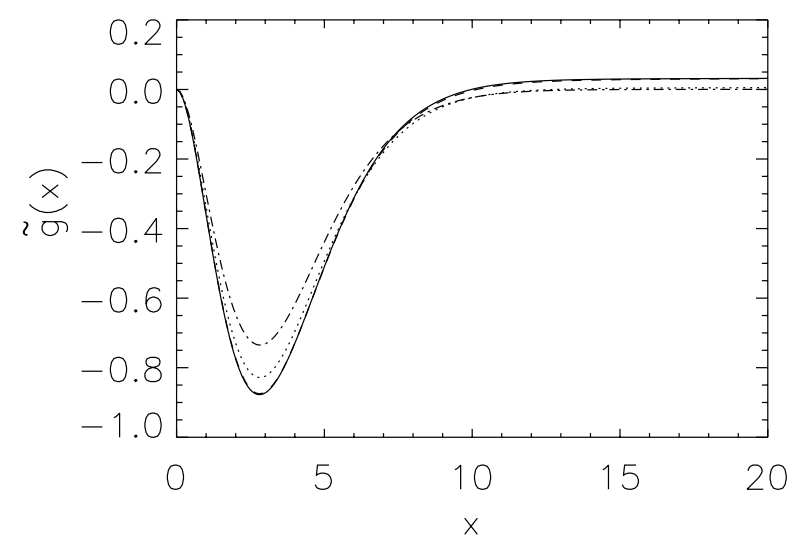

Fig. 12. The function $\tilde{g}(x)$ (see Eq. (45)) for non-thermal populations as in Eq. (34) with $p_{1}=0.5$ (solid line), 1 (dashes), 10 (dotted) and 100 (dot-dashes).

due to the large frequency shifts experienced by the CMB photons scattering the high energy non-thermal electrons.

We finally show in Fig. 14 the spectral distortion $\Delta i(x)$ for different values of $p_{1}$. Note, finally, that the amplitude of $\Delta I(x)$ produced by an electron population with a double power-law spectrum as in Eq. (34) is much lower than that produced by a population with a single power-law spectrum as in Eq. (32).

The case of a system dominated by non-thermal electrons is not applicable straightforwardly to galaxy clusters but rather to radio galaxies whose jets inject in the ICM large quantities of relativistic non-thermal electrons. We want to stress that our general approach is able to describe the Compton scattering distortions even in the cases of the radio lobes and of the jets of radio galaxies or AGNs with large optical depths and ultrarelativistic electron energies. We will address more specifically this issue in a forthcoming paper.

\section{The total SZ effect produced by a combination of thermal and non-thermal populations}

For galaxy clusters which contain two (or more) different electronic populations, like radio-halo clusters which contain a thermal population emitting X-rays and a non-thermal population producing the radio halo emission, one has to evaluate the spectral distortion produced simultaneously by both populations on the CMB radiation. Such a derivation has been not done so far. We assume here that the two populations are independent and that no change on the thermal population is induced by the non-thermal electrons. This condition is reasonable for non-thermal electrons with energies $\gtrsim 150 \mathrm{MeV}$ which do not appreciably loose energy through Coulomb collisions in the ICM (see, e.g., Blasi \& Colafrancesco 1999; Colafrancesco \& Mele 2001) and hence do not produce a substantial heating of the IC gas. Electrons with $E_{\mathrm{e}} \gtrsim 150 \mathrm{MeV}$ loose energy mainly through ICS and synchrotron losses. The radio halo emission in galaxy clusters is, in fact, produced by electrons with energy $E_{\mathrm{e}} \approx 16.4 \mathrm{GeV}(\mathrm{B} / \mu \mathrm{G})^{-1 / 2}\left(\nu_{\mathrm{r}} / \mathrm{GHz}\right)^{1 / 2}$ (see, e.g., Colafrancesco \& Mele 2001) which yield $E_{\mathrm{e}} \sim 1.6-52 \mathrm{GeV}$ for a typical IC magnetic field at the level of $B=1 \mu \mathrm{G}$ at the typical frequencies, $v_{\mathrm{r}} \sim 10^{-2}-10 \mathrm{GHz}$, at which radio halo spectra are observed. For such energies the non-thermal electrons do not strongly affect the thermal IC gas. However, we discuss in the following the impact of different non-thermal population spectra on both the total SZ effect and on the cluster IC gas.

Under the hypothesis of independence between the thermal and non-thermal electron populations, the probability that a CMB photon is scattered by an electron of population $\mathrm{A}$ (say thermal) is not affected by the fact that the same photon has been scattered by an electron of population B (say nonthermal). Thus, at first order in $\tau$ we expect that the total SZ effect is given by the sum of the first order SZ effects due to each single population. At higher order approximation in $\tau$, however, one has to consider the effect of repeated scattering (see Sect.2). It is intuitive to think that in this last case the total SZ effect is not simply given by the sum of the separate SZ effects at higher orders in $\tau$ and the terms describing the crossscattering between CMB photons and the electrons of populations A and B have to be taken into account. In the following, we will derive in detail the general expression for the total SZ distortion caused by a combination of two electronic populations.

Let us consider two electron populations with momentum distributions $f_{\mathrm{A}}(p)$ and $f_{\mathrm{B}}(p)$ with optical depths $\tau_{\mathrm{A}}$ and $\tau_{\mathrm{B}}$, respectively. We also assume that the two electron distributions are both normalized to 1 . The total distribution of the electron momenta is

$f_{\mathrm{e}}(p)=c_{\mathrm{A}} f_{\mathrm{A}}(p)+c_{\mathrm{B}} f_{\mathrm{B}}(p)$

with appropriate normalization coefficients $c_{\mathrm{A}}$ and $c_{\mathrm{B}}$. Since the total distribution $f_{\mathrm{e}}(p)$ is also normalized to 1 , the coefficients $c_{\mathrm{A}}$ and $c_{\mathrm{B}}$ are related by the condition $c_{\mathrm{A}}+c_{\mathrm{B}}=1$. The coefficients $c_{\mathrm{A}}$ and $c_{\mathrm{B}}$ yield the weight of each population with respect to the other and thus their ratio is given by

$\frac{c_{\mathrm{A}}}{c_{\mathrm{B}}}=\frac{\tau_{\mathrm{A}}}{\tau_{\mathrm{B}}}$.

Such a condition, together with the normalization condition, yields:

$c_{\mathrm{A}}=\frac{\tau_{\mathrm{A}}}{\tau_{\mathrm{A}}+\tau_{\mathrm{B}}} \quad c_{\mathrm{B}}=\frac{\tau_{\mathrm{B}}}{\tau_{\mathrm{A}}+\tau_{\mathrm{B}}}$.

The total distribution $f_{\mathrm{e}}(p)$ has an optical depth given by

$\tau=\tau_{\mathrm{A}}+\tau_{\mathrm{B}}$. 

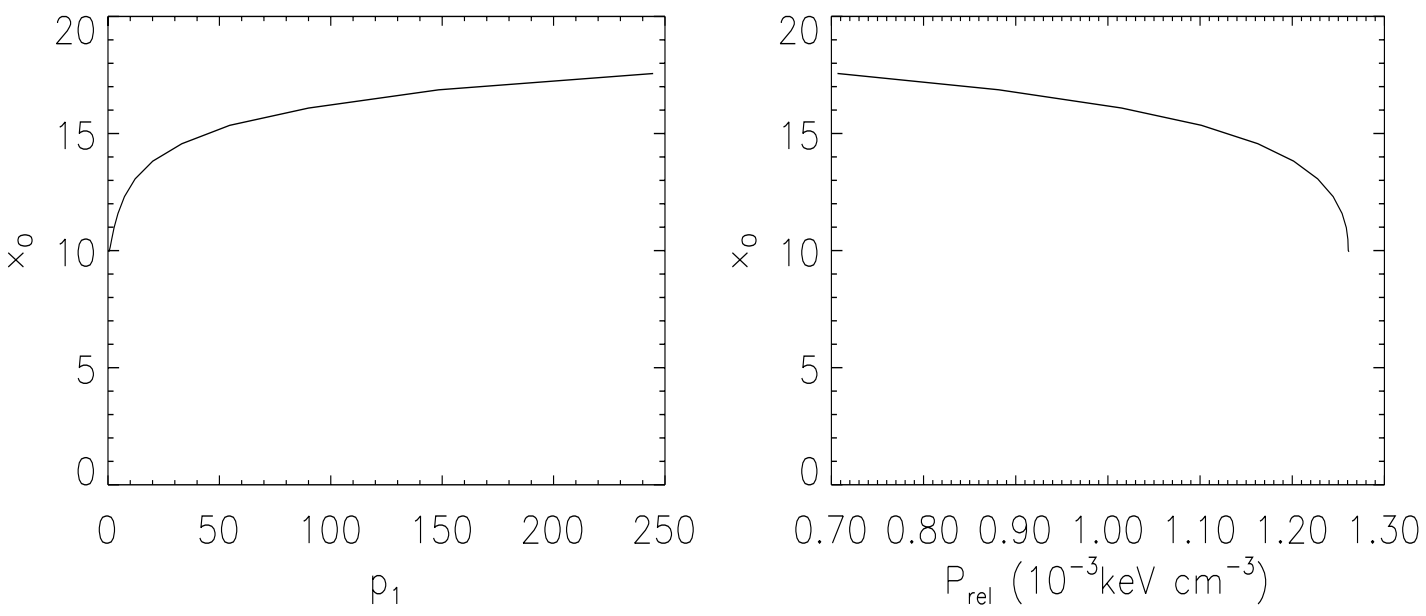

Fig. 13. The behaviour of the zero of the SZ effect for a non-thermal population with spectrum given by Eq. (34) as a function of $p_{1}$ (left panel) and of the pressure $P_{\text {rel }}$ (right panel) in units of $\mathrm{keV} \mathrm{cm}^{-3}$. As in Fig. 9, the pressure $P_{\text {rel }}$ decreases for increasing values of $p_{1}$ but with a different rate depending on the different spectrum of the electron distribution.

In fact, the probability that a CMB photon can suffer $n$ scatterings by the electrons of each distribution is given by the sum of all the possible combinations of the probability to suffer $n_{\mathrm{A}}$ scatterings from the electrons of the distribution $f_{\mathrm{A}}$ and the probability to suffer $n_{\mathrm{B}}$ scattering from the electrons of the distribution $f_{\mathrm{B}}$, with $n_{\mathrm{A}}+n_{\mathrm{B}}=n_{\mathrm{e}}$. Since the two distributions are independent, this probability is given by the product of the two separate probabilities:

$$
\begin{aligned}
p_{n} & =\sum_{n_{\mathrm{A}}+n_{\mathrm{B}}=n} p_{n_{\mathrm{A}}} \cdot p_{n_{\mathrm{B}}}=\sum_{n_{\mathrm{A}}+n_{\mathrm{B}}=n} \frac{\mathrm{e}^{-\tau_{\mathrm{A}}} \tau_{\mathrm{A}}^{n_{\mathrm{A}}}}{n_{\mathrm{A}} !} \cdot \frac{\mathrm{e}^{-\tau_{\mathrm{B}}} \tau_{\mathrm{B}}^{n_{\mathrm{B}}}}{n_{\mathrm{B}} !} \\
& =\sum_{n_{\mathrm{A}}+n_{\mathrm{B}}=n} \frac{\mathrm{e}^{-\left(\tau_{\mathrm{A}}+\tau_{\mathrm{B}}\right)} \tau_{\mathrm{A}}^{n_{\mathrm{A}}} \tau_{\mathrm{B}}^{n_{\mathrm{B}}}}{n_{\mathrm{A}} ! n_{\mathrm{B}} !}=\sum_{n_{\mathrm{A}}=0}^{n} \frac{\mathrm{e}^{-\left(\tau_{\mathrm{A}}+\tau_{\mathrm{B}}\right)} \tau_{\mathrm{A}}^{n_{\mathrm{A}}} \tau_{\mathrm{B}}^{n-n_{\mathrm{A}}}}{n_{\mathrm{A}} !\left(n-n_{\mathrm{A}}\right) !} \\
& =\mathrm{e}^{-\left(\tau_{\mathrm{A}}+\tau_{\mathrm{B}}\right)} \sum_{n_{\mathrm{A}}=0}^{n} \frac{1}{n !} \frac{n !}{n_{\mathrm{A}} !\left(n-n_{\mathrm{A}}\right) !} \tau_{\mathrm{A}}^{n_{\mathrm{A}}} \tau_{\mathrm{B}}^{n-n_{\mathrm{A}}} \\
& =\frac{\mathrm{e}^{-\left(\tau_{\mathrm{A}}+\tau_{\mathrm{B}}\right)}\left(\tau_{\mathrm{A}}+\tau_{\mathrm{B}}\right)^{n}}{n !} \equiv \frac{\mathrm{e}^{-\tau} \tau^{n}}{n !}
\end{aligned}
$$

(we assume here a Poisson probability distribution). This means that the electrons of the distribution $f_{\mathrm{B}}$ simply add to the electrons of the distribution $f_{\mathrm{A}}$. In fact, from the definition of optical depth, one gets:

$\tau=\sigma_{\mathrm{T}} \int n_{\mathrm{e}} \mathrm{d} \ell \equiv \tau_{\mathrm{A}}+\tau_{\mathrm{B}}=\sigma_{\mathrm{T}} \int\left(n_{\mathrm{A}}+n_{\mathrm{B}}\right) \mathrm{d} \ell$.

The exact expression for the spectral distortion produced by two electron populations can be evaluated from Eqs. (19)-(21), using the appropriate expression for $P_{1}(s)$. The general expression for the distribution $P_{1}(s)$ is given by:

$P_{1}(s)=\int_{0}^{\infty} \mathrm{d} p f_{\mathrm{e}}(p) P_{\mathrm{s}}(s ; p)$.

Inserting Eq. (52) in Eq. (58) one obtains the function $P_{1}(s)$ for two populations as a function of the distributions $P_{1}(s)$ of each single population:

$$
\begin{aligned}
P_{1}(s) & =c_{\mathrm{A}} \int_{0}^{\infty} \mathrm{d} p f_{\mathrm{A}}(p) P_{\mathrm{s}}(s ; p)+c_{\mathrm{B}} \int_{0}^{\infty} \mathrm{d} p f_{\mathrm{B}}(p) P_{\mathrm{s}}(s ; p) \\
& \equiv c_{\mathrm{A}} P_{1 \mathrm{~A}}(s)+c_{\mathrm{B}} P_{1 \mathrm{~B}}(s) .
\end{aligned}
$$

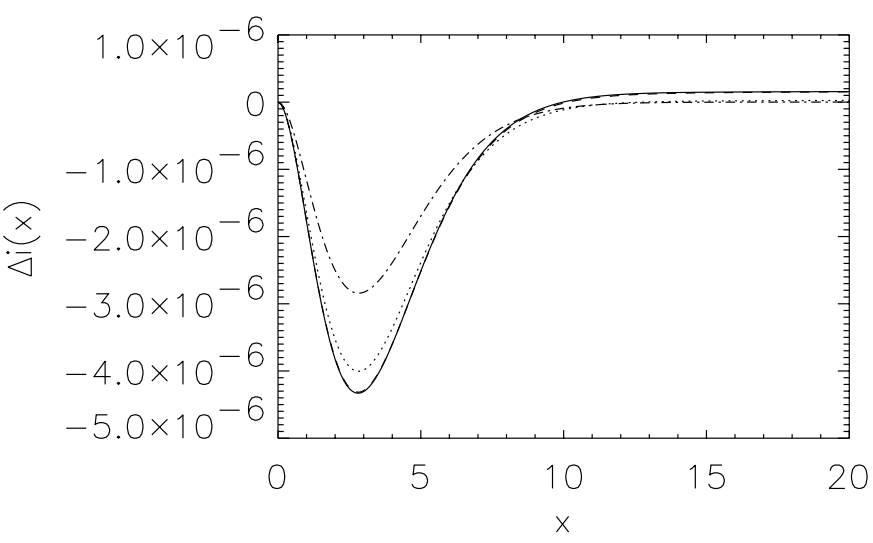

Fig. 14. The spectral distortion in units of $2\left(k_{\mathrm{B}} T_{0}\right)^{3} /(h c)^{2}$ for a nonthermal population given by Eq. (34) computed for $p_{1}=0.5$ (solid), 1 (dashes), 10 (dotted) and 100 (dot-dashes).

The Fourier transform of Eq. (59) is

$\tilde{P}_{1}(k)=c_{\mathrm{A}} \tilde{P}_{1 \mathrm{~A}}(k)+c_{\mathrm{B}} \tilde{P}_{1 \mathrm{~B}}(k)$

and from this expression one obtains the Fourier transform of the total redistribution function:

$$
\begin{aligned}
\tilde{P}(k) & =\mathrm{e}^{-\tau\left[1-\tilde{P}_{1}(k)\right]} \\
& =\mathrm{e}^{-\left(\tau_{\mathrm{A}}+\tau_{\mathrm{B}}\right)\left[1-\frac{\tau_{\mathrm{A}}}{\tau_{\mathrm{A}}+\tau_{\mathrm{B}}} \tilde{P}_{1 \mathrm{~A}}(k)-\frac{\tau_{\mathrm{B}}}{\tau_{\mathrm{A}}+\tau_{\mathrm{B}}} \tilde{P}_{1 \mathrm{~B}}(k)\right]} \\
& =\mathrm{e}^{-\tau_{\mathrm{A}}\left[1-\tilde{P}_{1 \mathrm{~A}}(k)\right]-\tau_{\mathrm{B}}\left[1-\tilde{P}_{1 \mathrm{~B}}(k)\right]} \\
& =\tilde{P}_{\mathrm{A}}(k) \tilde{P}_{\mathrm{B}}(k) .
\end{aligned}
$$

The exact total redistribution function $P_{\text {tot }}(s)$ is given by the convolution of the redistribution functions of the separate electron populations:

$P_{\text {tot }}(s)=P_{\mathrm{A}}(s) \otimes P_{\mathrm{B}}(s)$.

Thus, the exact spectral distortion produced by two electron populations on the $\mathrm{CMB}$ radiation is given by:

$I_{\mathrm{tot}}(x)=\int_{-\infty}^{+\infty} I_{0}\left(x \mathrm{e}^{-s}\right) P_{\mathrm{tot}}(s) \mathrm{d} s$, 
in terms of the exact total redistribution function $P_{\text {tot }}(s)$ given in Eq. (62). We also give in the Appendix the analytic expressions for the approximations at first and second order in $\tau$ of the total spectral distortion $I_{\text {tot }}(x)$.

\subsection{The function $\tilde{g}(x)$ for a combination of two electron populations}

The total SZ effect produced by the combination of two electron populations can be written again in terms of a Comptonization parameter $y_{\text {tot }}$ and of a spectral function $\tilde{g}(x)$, in the form of Eq. (22). For two electron populations, we can write the average temperature in Eq. (46) as:

$$
\begin{aligned}
\left\langle k_{\mathrm{B}} T_{\mathrm{e}}\right\rangle & =\int_{0}^{\infty} \mathrm{d} p f_{\mathrm{e}}(p) \frac{1}{3} p v(p) m_{\mathrm{e}} c \\
& =\int_{0}^{\infty} \mathrm{d} p\left[c_{\mathrm{A}} f_{\mathrm{A}}(p)+c_{\mathrm{B}} f_{\mathrm{B}}(p)\right] \frac{1}{3} p v(p) m_{\mathrm{e}} c \\
& =c_{\mathrm{A}}\left\langle k_{\mathrm{B}} T_{\mathrm{e}}\right\rangle_{\mathrm{A}}+c_{\mathrm{B}}\left\langle k_{\mathrm{B}} T_{\mathrm{e}}\right\rangle_{\mathrm{B}} .
\end{aligned}
$$

The Comptonization parameter $y_{\text {tot }}$ can be evaluated using Eq. (49) as:

$$
\begin{aligned}
y_{\text {tot }} & =\frac{1}{m_{\mathrm{e}} c^{2}}\left[c_{\mathrm{A}}\left\langle k_{\mathrm{B}} T_{\mathrm{e}}\right\rangle_{\mathrm{A}}+c_{\mathrm{B}}\left\langle k_{\mathrm{B}} T_{\mathrm{e}}\right\rangle_{\mathrm{B}}\right]\left(\tau_{\mathrm{A}}+\tau_{\mathrm{B}}\right) \\
& =\frac{1}{m_{\mathrm{e}} c^{2}}\left[\tau_{\mathrm{A}}\left\langle k_{\mathrm{B}} T_{\mathrm{e}}\right\rangle_{\mathrm{A}}+\tau_{\mathrm{B}}\left\langle k_{\mathrm{B}} T_{\mathrm{e}}\right\rangle_{\mathrm{B}}\right]=y_{\mathrm{A}}+y_{\mathrm{B}} .
\end{aligned}
$$

At first order in $\tau$, the function $\tilde{g}(x)$ for two populations can be expressed as

$$
\begin{aligned}
\tilde{g}(x) & =\frac{\Delta i(x)}{y_{\mathrm{tot}}}=\frac{\tau_{\mathrm{A}}\left[j_{1 \mathrm{~A}}(x)-j_{0}(x)\right]+\tau_{\mathrm{B}}\left[j_{1 \mathrm{~B}}(x)-j_{0}(x)\right]}{y_{\mathrm{A}}+y_{\mathrm{B}}} \\
& =\frac{y_{\mathrm{A}} \tilde{g}_{\mathrm{A}}(x)+y_{\mathrm{B}} \tilde{g}_{\mathrm{B}}(x)}{y_{\mathrm{A}}+y_{\mathrm{B}}},
\end{aligned}
$$

in terms of the functions $\tilde{g}(x)$ for each single electron population. We notice that in this case the function $\tilde{g}(x)$ depends from the Comptonization parameters, and hence from the optical depths, of the two electron populations even at the first order approximation in $\tau$. Moreover, at higher orders in $\tau$, the function $\tilde{g}(x)$ cannot be expressed simply in terms of the spectral functions of the single populations because in the expression for $\Delta i(x)$ (see Eq. (A.10)), cross-correlation terms appear.

\subsection{The SZ effect produced by a combination of a thermal plus a non-thermal populations}

Here we apply the previous derivation of the total SZ effect to a galaxy cluster which contains both a termal and a nonthermal electron populations. In the following computation of the total SZ effect for a Coma-like cluster we choose a thermal population with the following parameters: $k_{\mathrm{B}} T_{\mathrm{e}}=8.5 \mathrm{keV}$, $n_{\mathrm{e}, \mathrm{th}} \simeq 3 \times 10^{-3} \mathrm{~cm}^{-3}$ and a cluster radius of $1 h_{50}^{-1} \mathrm{Mpc}$. With these parameters the optical depth of the thermal population is

$$
\tau_{\text {th }} \simeq 6 \times 10^{-3}\left[\frac{n_{\mathrm{e}, \mathrm{th}}}{3 \times 10^{-3} \mathrm{~cm}^{-3}}\right]\left[\frac{\ell}{1 h_{50}^{-1} \mathrm{Mpc}}\right],
$$

and its pressure is:

$$
P_{\text {th }} \simeq 2.55 \times 10^{-2} \mathrm{keV} \mathrm{cm}^{-3}\left[\frac{n_{\mathrm{e}, \mathrm{th}}}{3 \times 10^{-3} \mathrm{~cm}^{-3}}\right]\left[\frac{k_{\mathrm{B}} T_{\mathrm{e}}}{8.5 \mathrm{keV}}\right] \text {. }
$$

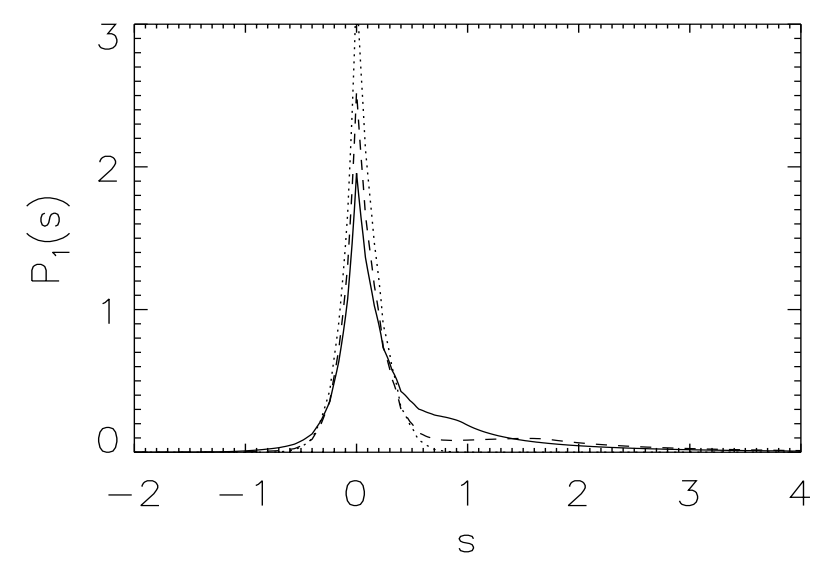

Fig. 15. The function $P_{1}(s)$ (see Eq. (59)) for a combination of thermal population and non-thermal population as in Eq. (32) with $p_{1}=$ 0.5 (solid), 1 (dashes) and 10 (dotted).

As for the spectrum of the non-thermal population we consider both the phenomenological cases of a single and double power-law populations (see Eq. (32) and Eq. (34) respectively). The parameters we consider are specifically: $\alpha=2.5$ and $p_{2} \rightarrow \infty$ for the single power-law population and $\alpha_{1}=0.5$, $\alpha_{2}=2.5$, with $p_{\text {cr }}=400$ and $p_{2} \rightarrow \infty$ for the double powerlaw population. We consider $p_{1}$ as a free parameter in both cases. The relativistic electron density has been normalized at $n_{\text {e,rel }}\left(\tilde{p}_{1}\right)=10^{-6} \mathrm{~cm}^{-3}$ for $\tilde{p}_{1}=100$ in both cases. The final SZ effect can be re-scaled simply to different values of $n_{\mathrm{e}, \text { rel }}$ as discussed in Sect. 3.

The amplitude of the non-thermal contribution to the total SZ effect increases with decreasing value of $p_{1}$. For the case of a single non-thermal power-law population it becomes appreciable for $p_{1} \lesssim 10$ while for $p_{1} \gtrsim 100$ the total SZ effect is indistinguishable from the pure thermal effect. In fact, for $p_{1} \gtrsim 10$ the function $P_{1}(s)$ is nearly coincident with that of a single thermal population as shown in Fig. 15, while for $p_{1}<10$ the function $P_{1}(s)$ is more a-symmetric towards large and positive values of $s$. The peaks of the non-thermal distributions at large $s$ are present in the total distribution $P_{1}(s)$ but their amplitude decreases with $\tau_{\text {rel }}$ and becomes lower and lower with increasing values of $p_{1}$.

The amplitude and the shape of the total SZ effect in a cluster which contains non-thermal phenomena depends on the spectral and spatial distribution of the non-thermal population, given that the cluster IC gas properties are well known from $\mathrm{X}$-ray observations. We can take advantage of this fact to use the non-thermal SZ effect as a tool to constrain the physical properties of the non-thermal population. We show in Fig. 16 the total spectral distortion due to the SZ effect in a cluster with non-thermal population as a function of $p_{1}$. Low values of $p_{1} \lesssim 1$ for the non-thermal population induce large distortions with respect to the pure thermal SZ effect. However, for low values of $p_{1}$ the pressure of the non-thermal population with single power-law distribution exceeds substantially the thermal pressure $P_{\text {th }}$ (see Table 7); as a consequence, the low energy electrons produce an exceedingly large pressure unbalance and also a large heating of the IC gas, which are not acceptable. 

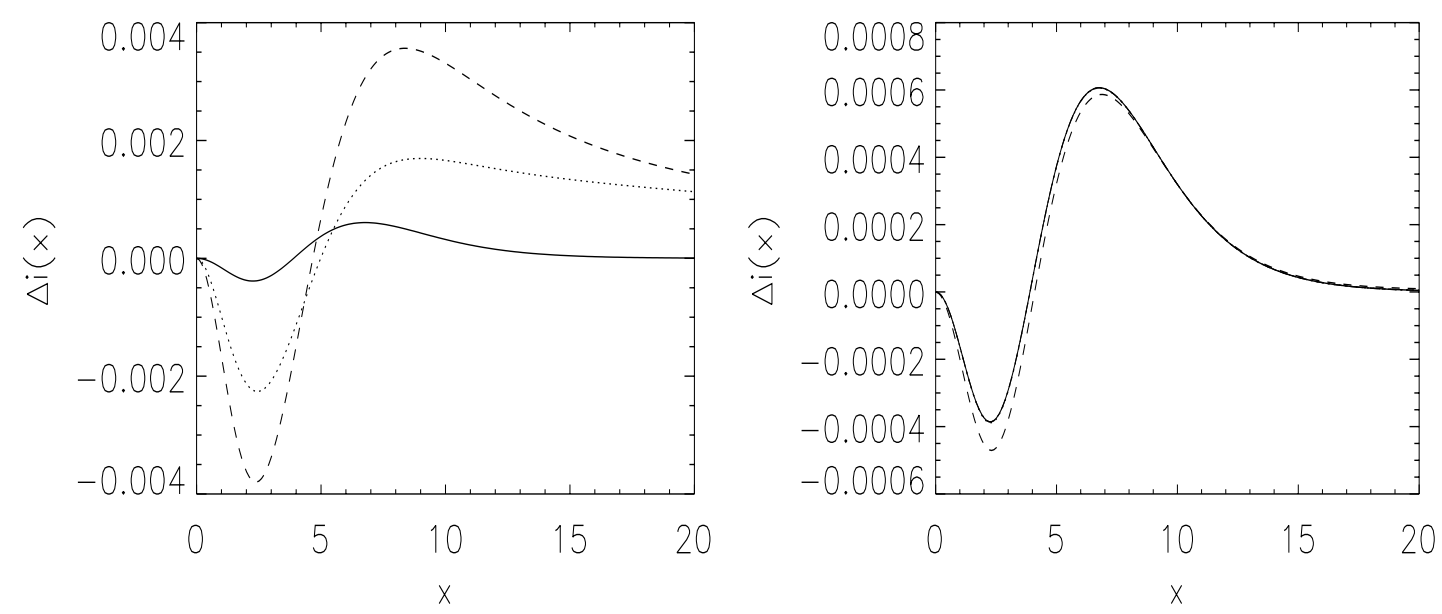

Fig. 16. The spectral distortion, in units of $2\left(k_{\mathrm{B}} T_{0}\right)^{3} /(h c)^{2}$, of a single thermal population of a Coma-like cluster (solid) is compared with the spectral distortion evaluated at first order in $\tau$ (see Eq. (A.6)), produced by a combination of thermal and non-thermal population given in Eq. (32) with $p_{1}=0.5$ (dashes) and 1 (dotted) (left panel) and for $p_{1}=10$ (dashes), 100 (dotted) and 1000 (dot-dashes) (right panel).
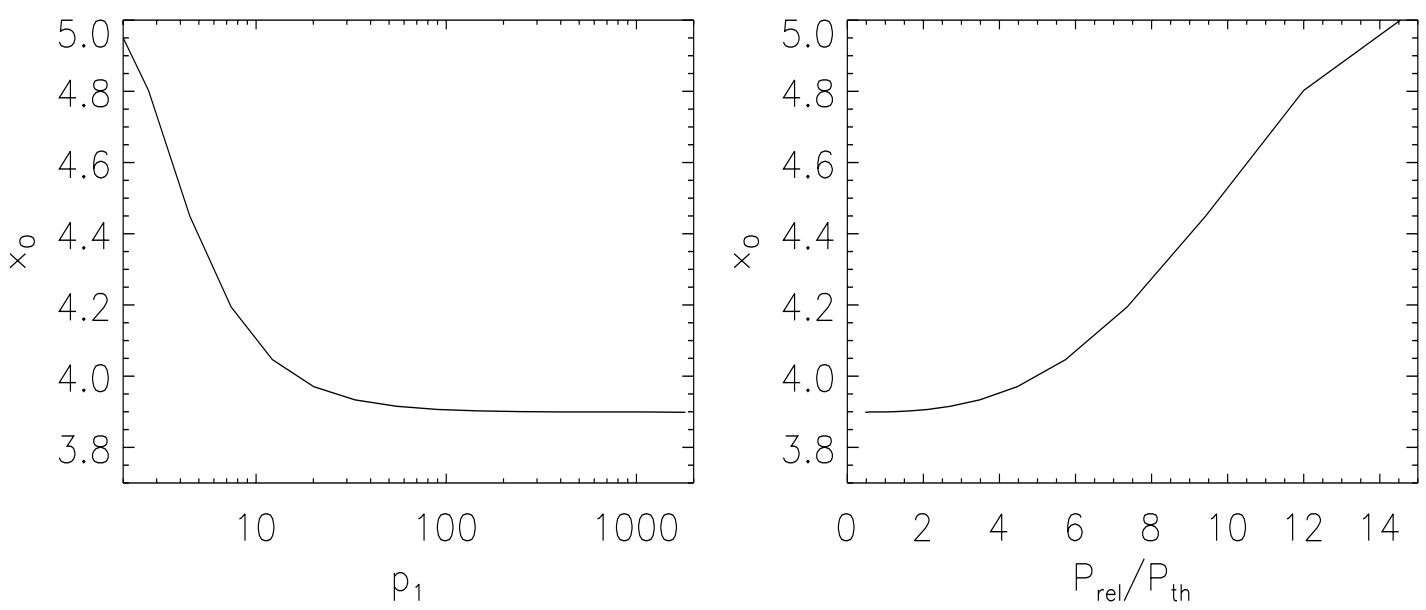

Fig. 17. The behaviour of the zero of the total SZ effect evaluated for a combination of thermal and non-thermal population given by Eq. (32) is shown as a function of minimum momentum, $p_{1}$, of the non-thermal population (left) and of the pressure ratio $P_{\text {rel }} / P_{\text {th }}$ (right).

Table 7. Values of pressure and density ratios for a combination of thermal population and a non-thermal population with single powerlaw spectrum for different values of $p_{1}$ (see text for details).

\begin{tabular}{ccc}
\hline \hline$p_{1}$ & $P_{\text {rel }} / P_{\text {th }}$ & $n_{\mathrm{e}, \text { th }} / n_{\mathrm{e}, \text { rel }}$ \\
\hline 0.5 & 23.3 & 1.06 \\
1 & 18.6 & 3.00 \\
10 & 6.33 & 94.87 \\
100 & 2.00 & 3000 \\
1000 & 0.63 & 94868 \\
\hline
\end{tabular}

The frequency location of the zero of the total SZ effect, $x_{0}$, is also a powerful diagnostic of the presence and of the nature of the non-thermal population. The frequency location of $x_{0}$ increases for decreasing values of $p_{1}$ as the impact of the non-thermal population becomes larger (see Fig. 17). Consistently, $x_{0}$ increases with increasing values of the pressure ratio $P_{\text {rel }} / P_{\text {th }}$, as also shown in Fig. 17.

In the case of a double power-law non-thermal population the ratio $P_{\text {rel }} / P_{\text {th }}$ always remains of order $10^{-2}$ even for vary
Table 8. Values of pressure and density ratio for a thermal population and a non-thermal population with double power-law spectrum for different values of $p_{1}$ (see text for details).

\begin{tabular}{ccc}
\hline \hline$p_{1}$ & $P_{\text {rel }} / P_{\text {th }}$ & $n_{\text {e,th }} / n_{\text {e,rel }}$ \\
\hline 0.5 & $4.94 \times 10^{-2}$ & 1926 \\
1 & $4.94 \times 10^{-2}$ & 1948 \\
10 & $4.84 \times 10^{-2}$ & 2127 \\
100 & $3.87 \times 10^{-2}$ & 3000 \\
\hline
\end{tabular}

low values $p_{1} \sim 0.5$ (see Table 8 ), and in this case there is a negligible dynamical and thermal influence of the nonthermal population on the thermal one. In this case, also the total distribution $P_{1}(s)$ and the total spectral distortion are little affected by the value of $p_{1}$. We show in Fig. 18 the spectral distortion of a single thermal population compared with the spectral distortion produced by a combination of thermal and non-thermal population with double power-law spectrum. The contribution of the non-thermal population to the total SZ effect depends on the normalization of the density $n_{\mathrm{e}, \text { rel }}$ 

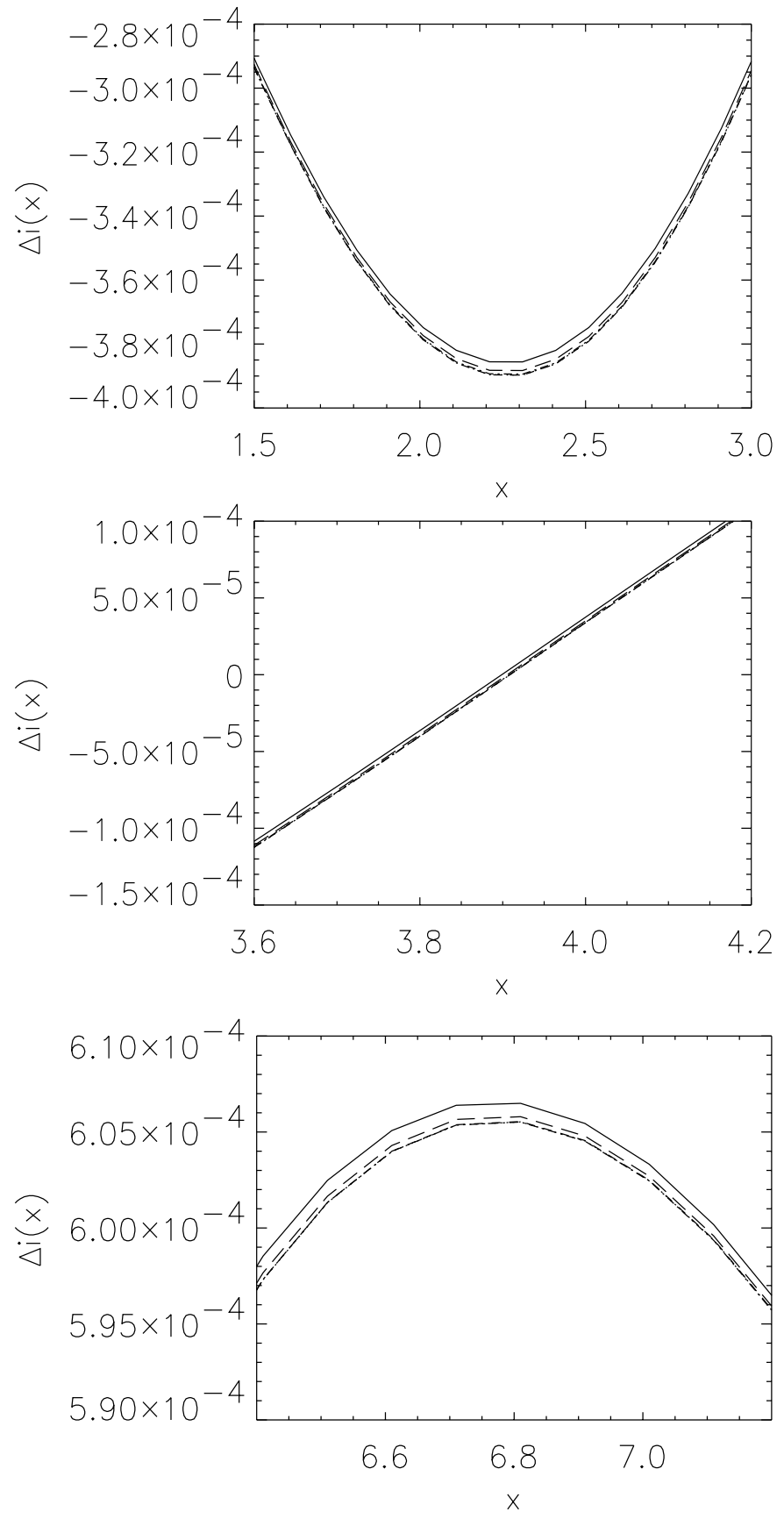

Fig. 18. The spectral distortion, in units of $2\left(k_{\mathrm{B}} T_{0}\right)^{3} /(h c)^{2}$, of a single thermal population (solid) is compared with the spectral distortion evaluated at first order in $\tau$ produced by a combination of thermal and non-thermal population given in Eq. (34) with $n_{\mathrm{e}, \text { rel }}\left(\tilde{p}_{1}=100\right)=$ $1 \times 10^{-6}$ and $p_{1}=0.5$ (dashes), 1 (dotted), 10 (dot-dashes) and 100 (long dashes). We show the enlargement in the regions of the minimum (top), of the zero (mid) and of the maximum (bottom) of the total SZ effect.

(which is highly uncertain). For increasing values of the density of the non-thermal population the non-thermal SZ effect becomes more relevant and produces substantial changes to the SZ distortion produced by a single thermal population at frequencies $x \lesssim 10$ (see Fig. 19). Much milder changes are present in the high frequency range $x \gtrsim 15$ due to the low
Table 9. Values of pressure and density ratio and the corresponding position of the zero for a thermal population and a non-thermal double power-law population for $p_{1}=0.5$ as a function of $n_{\mathrm{e}, \text { rel }}\left(\tilde{p}_{1}=100\right)$ (see text for details).

\begin{tabular}{cccc}
\hline \hline$n_{\mathrm{e}, \text { rel }}\left(\tilde{p}_{1}=100\right)\left(\mathrm{cm}^{-3}\right)$ & $P_{\text {rel }} / P_{\text {th }}$ & $n_{\mathrm{e}, \mathrm{th}} / n_{\mathrm{e}, \mathrm{rel}}$ & $x_{0}$ \\
\hline $1 \times 10^{-6}$ & $4.94 \times 10^{-2}$ & 1926 & 3.9085 \\
$1 \times 10^{-5}$ & 0.49 & 192 & 3.9965 \\
$3 \times 10^{-5}$ & 1.48 & 64 & 4.1765 \\
\hline
\end{tabular}

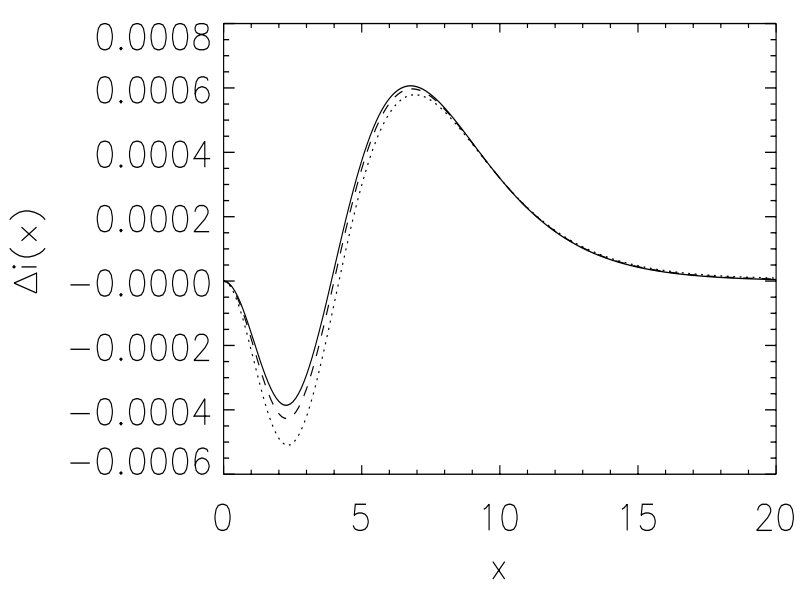

Fig. 19. The spectral distortion, in units of $2\left(k_{\mathrm{B}} T_{0}\right)^{3} /(h c)^{2}$, of a single thermal population (solid) is compared with the spectral distortion evaluated at first order in $\tau$ produced by a combination of thermal and non-thermal population given in Eq. (34) with $p_{1}=0.5$ and $n_{\mathrm{e}}\left(\tilde{p}_{1}=100\right)=10^{-5}$ (dashes) and $3 \times 10^{-5} \mathrm{~cm}^{-3}$ (dotted).

amplitude of the non-thermal signal in this region (see, e.g., Fig. 14). We also show in Fig. 20 the behaviour of $x_{0}$ as a function of $p_{1}$ and of the pressure ratio for a density normalization $n_{\mathrm{e}, \text { rel }}\left(\tilde{p}_{1}=100\right)=3 \times 10^{-5} \mathrm{~cm}^{-3}$. High values of the ratio $P_{\text {rel }} / P_{\text {th }}$ produce a substantial displacement of $x_{0}$ up to values $x_{0} \gtrsim 4$ which correspond to frequencies $\gtrsim 227 \mathrm{GHz}$. In Fig. 21 we also show the behaviour of $x_{0}$ a a function of $n_{\mathrm{e}, \mathrm{rel}}\left(\tilde{p}_{1}=100\right)$ for $p_{1}=0.5$. We also report in Table 9 the values of the density and the corresponding values of $P_{\text {rel }} / P_{\text {th }}$ and of $n_{\mathrm{e}, \mathrm{th}} / n_{\mathrm{e}, \text { rel }}$ as well as the frequency position of the zero of the total SZ effect. It is interesting to note in this context that the precise detection of the frequency location of $x_{0}$ provides a direct measure of the ratio $P_{\text {rel }} / P_{\text {th }}$ and, in turn, of the energy density and of the properties of the spectrum of the nonthermal population once the pressure of the thermal population is known from X-ray observations. In fact, all these physical information contributes to set the specific amplitude and the spectral shape of the total SZ effect (see Figs. 17 and 20 and Figs. 16, 18 and 20 for the different SZ effect features produced by two different non-thermal electron populations). This is a relevant and unique feature of the non-thermal SZ effect in clusters since it yields a measure of the total pressure in relativistic non-thermal particles in the cluster atmosphere, an information which is not easily accessible from the study of other non-thermal phenomena like radio halos and/or EUV or hard X-ray excesses. Thus, the detailed observations of the frequency shift of $x_{0}$ in clusters provides unambiguously relevant 
Table 10. Values of pressure and density ratios and the corresponding position of the zero for a combination of two thermal populations with $k_{\mathrm{B}} T_{\mathrm{e}, 1}=8.5$ and $k_{\mathrm{B}} T_{\mathrm{e}, 2}=1 \mathrm{keV}$ for different values of $n_{\mathrm{e}, 2}$. A value $n_{\mathrm{e}, 1}=3 \times 10^{-3} \mathrm{~cm}^{-3}$ is adopted here.

\begin{tabular}{cccc}
\hline \hline$n_{\mathrm{e}, 2}\left(\mathrm{~cm}^{-3}\right)$ & $P_{2} / P_{1}$ & $n_{\mathrm{e}, 1} / n_{\mathrm{e}, 2}$ & $x_{0}$ \\
\hline $1 \times 10^{-3}$ & $3.92 \times 10^{-2}$ & 3 & 3.8955 \\
$3 \times 10^{-3}$ & 0.12 & 1 & 3.8945 \\
$5 \times 10^{-3}$ & 0.20 & 0.6 & 3.8895 \\
$7 \times 10^{-3}$ & 0.27 & 0.43 & 3.8855 \\
$1 \times 10^{-2}$ & 0.39 & 0.3 & 3.8805 \\
\hline
\end{tabular}

constraints to the relativistic particle content in the ICM and to their energy distribution. Such a measurement is also crucial to determine the true amount of kinematic SZ effect due to the cluster peculiar velocity since it is usually estimated from the residual SZ signal at the location of the zero of the thermal (relativistic) SZ effect. Due to the steepness of the SZ spectral shape in the region of the null of the overall SZ effect, the possible additional non-thermal SZ signal must be determined precisely in order to derive reliable limits to the kinematic SZ effect. We will present a more detailed analysis of the kinematic SZ effect in a forthcoming paper.

\section{The SZ effect produced by a combination of different thermal populations.}

The general derivation of the total SZ effect produced by different electron populations described in Sect. 4 above can be applied also to the case of a combination of different thermal populations. So, in this Section we consider the SZ effect produced by two thermal populations with different temperatures and densities residing in the same cluster. The presence of an additional thermal IC population of quite low temperature, $T \lesssim 1 \mathrm{keV}$, has been considered as a possibility to explain the origin of the EUV/soft X-ray excess observed in some nearby clusters (Coma and Virgo for instance, see e.g. Lieu et al. 1999) and it is also expected in the outer regions of the clusters due to infall of warm gas from large scale filaments. Here we want to explore the modifications that such additional warm component can introduce into the overall SZ effect in galaxy clusters. More interestingly, we want to show that is possible to obtain constraints on any additional thermal component in galaxy clusters using SZ observations. We consider for this additional thermal population temperatures in the range $k_{\mathrm{B}} T_{\mathrm{e}, 2}=0.5-1 \mathrm{keV}$ and densities $n_{\mathrm{e}, 2}$ in the range $10^{-3}-10^{-2} \mathrm{~cm}^{-3}$. We also assume here, for simplicity, that the two thermal populations have the same spatial distribution. Moreover, we report in Tables 10 and 11 the pressure ratio, the density ratio and the frequency location of the zero of the total SZ effect for two values of the temperature of the warm population: $k_{\mathrm{B}} T_{\mathrm{e}, 2}=0.5$ and $1 \mathrm{keV}$, respectively. The presence of additional cooler electrons produces a tightening of the photon re-distribution function $P_{1}(s)$ (see Fig. 22) around $s=0$. At the same time, the total optical depth increases and, as a consequence, the total spectral distortion shows a deeper minimum and a higher maximum with respect to the single thermal population case (see Fig. 23). It is
Table 11. Values of pressure and density ratio and the corresponding position of the zero for a combination of two thermal populations with $k_{\mathrm{B}} T_{\mathrm{e}, 1}=8.5$ and $k_{\mathrm{B}} T_{\mathrm{e}, 2}=0.5 \mathrm{keV}$ for different values of $n_{\mathrm{e}, 2}$. A value $n_{\mathrm{e}, 1}=3 \times 10^{-3} \mathrm{~cm}^{-3}$ is adopted here.

\begin{tabular}{cccc}
\hline \hline$n_{\mathrm{e}, 2}\left(\mathrm{~cm}^{-3}\right)$ & $P_{2} / P_{1}$ & $n_{\mathrm{e}, 1} / n_{\mathrm{e}, 2}$ & $x_{0}$ \\
\hline $1 \times 10^{-3}$ & $1.96 \times 10^{-2}$ & 3 & 3.9005 \\
$3 \times 10^{-3}$ & $5.88 \times 10^{-2}$ & 1 & 3.8965 \\
$5 \times 10^{-3}$ & $9.80 \times 10^{-2}$ & 0.6 & 3.8935 \\
$7 \times 10^{-3}$ & 0.14 & 0.43 & 3.8895 \\
$1 \times 10^{-2}$ & 0.20 & 0.3 & 3.8855 \\
\hline
\end{tabular}

evident that high values of the density of the cooler population can introduce substantial changes to the total SZ effect.

We also show in Fig. 24 the variation of the zero of the total SZ effect as a function of the density of the cooler population and of the pressure ratio $P_{2} / P_{1}$, respectively. As the cooler population becomes more relevant (i.e., for increasing values of its pressure $P_{2}$ ), the position of $x_{0}$ moves towards lower frequency values, as expected. In Fig. 25 the behaviour of $x_{0}$ is shown as a function of the temperature of the cooler population and of the pressure ratio $P_{2} / P_{1}$ for a fiducial value of the density $n_{\mathrm{e}, 2}=1 \times 10^{-3} \mathrm{~cm}^{-3}$.

\section{The spatial distribution of the total SZ effect}

In this section we want to study the effect of the superposition of two distinct electron populations on the spatial distribution of the total SZ effect in galaxy clusters. In fact, the thermal and the non-thermal populations have, in general, a different spatial distribution as indicated by the spatial extension of the $\mathrm{X}$-ray emission and of the radio halo emission in many clusters (see Giovannini et al. 2001). Also, there are indications that the EUV emission in some nearby clusters is more extended than the thermal X-ray emission suggesting that there is a warm gas and/or a population of relativistic electrons which are spatially more extended than the X-ray emitting IC gas (Lieu et al. 1999).

Let us first consider a galaxy cluster in which two different electron populations are present: a thermal population, described by a relativistic Maxwellian velocity distribution, and a non-thermal population, with an energy spectrum described by a double power-law (see Eq. (34)). We consider these two populations as independent and spatially superposed, consistently with our analysis of Sect. 4 . These assumptions are indeed reasonable since in the cluster A2163, for instance, the radio halo emitting electrons occupy approximately the same volume of the X-ray emitting, thermal electrons (see Feretti et al. 2001) and, moreover, the relativistic electrons with $E_{\mathrm{e}} \sim$ a few $\mathrm{GeV}$ (which produce the radio-halo synchrotron emission) do not sensitively affect the IC thermal electrons.

As for the spatial distribution of the thermal electrons we use here a isothermal $\beta$-model (see, e.g., Cavaliere \& FuscoFemiano 1976), according to which the temperature $T_{\mathrm{e}}$ of the 

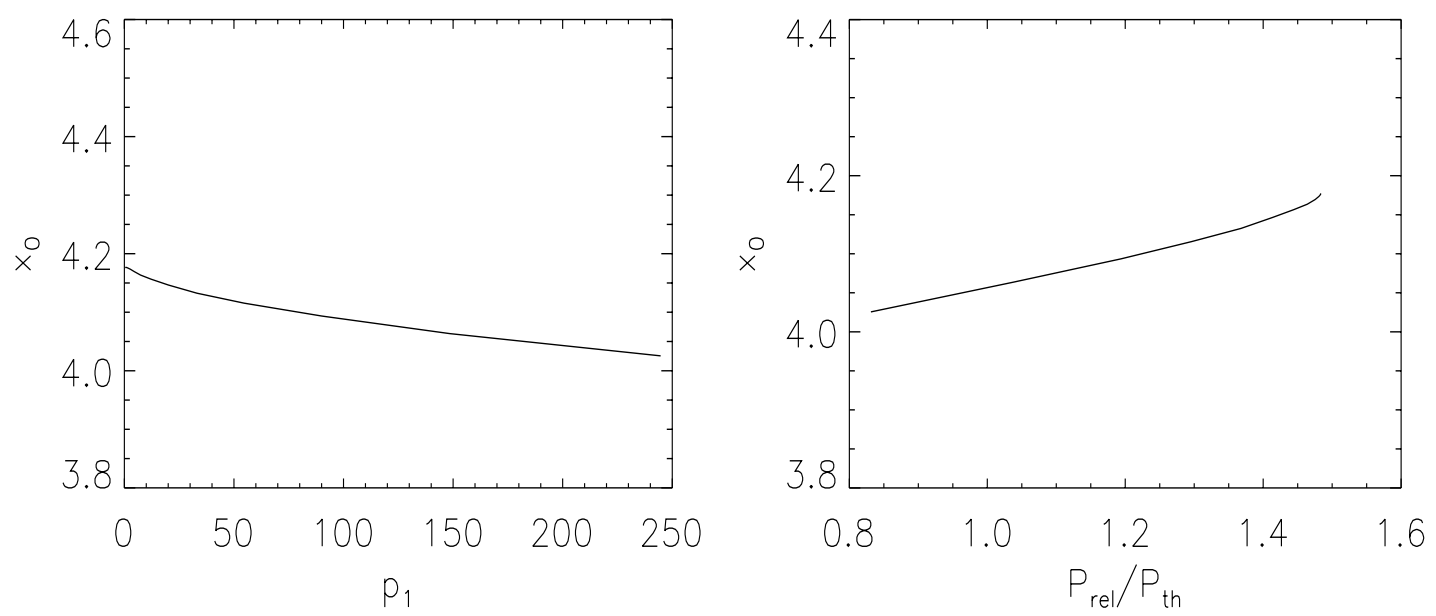

Fig. 20. The behaviour of the zero of the total $\mathrm{SZ}$ effect produced by a combination of a thermal and non-thermal population given in Eq. (34) with $n_{\mathrm{e}, \text { rel }}\left(\tilde{p}_{1}=100\right)=3 \times 10^{-5} \mathrm{~cm}^{-3}$ as a function of $p_{1}($ left $)$ and of the pressure ratio $P_{\text {rel }} / P_{\text {th }}$ (right).

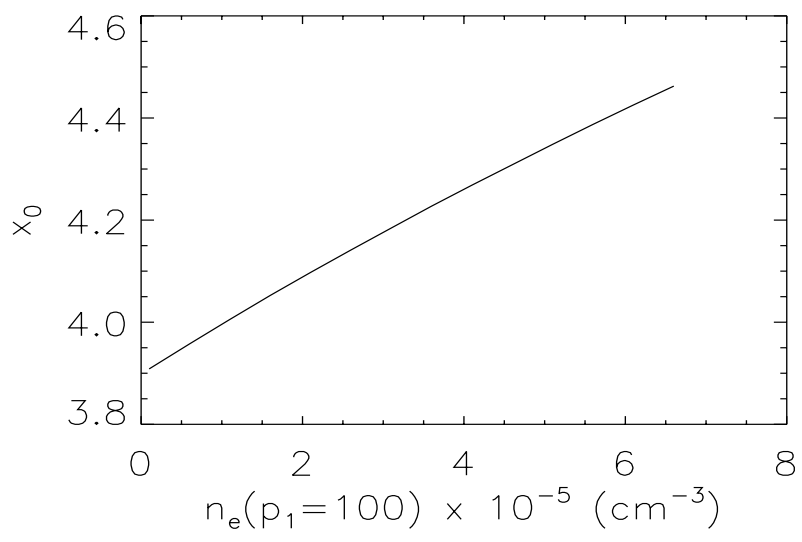

Fig. 21. The behaviour of the zero of the total SZ effect produced by a combination of a thermal and non-thermal population given in Eq. (34) with $p_{1}=0.5$ is shown as a function of $n_{\text {e,rel }}\left(\tilde{p}_{1}=100\right)$.

ICM is constant and the electron density is described by a spherical distribution

$n_{\mathrm{e}}(r)=n_{\mathrm{e} 0}\left(1+\frac{r^{2}}{r_{\mathrm{c}}^{2}}\right)^{-3 \beta / 2}$,

where $n_{\mathrm{e} 0}$ is the central IC gas density, $r_{\mathrm{c}}$ is the core radius of the cluster and $\beta=\mu m_{\mathrm{p}} v^{2} / k_{\mathrm{B}} T_{\mathrm{e}}$ is observed in the range $0.6-1$ (see Sarazin 1988 for a review). Under this assumption the angular dependence of the optical depth of the thermal population is given by

$$
\begin{aligned}
\tau(\theta) & =\sigma_{\mathrm{T}} \int n_{\mathrm{e}}(r) \mathrm{d} \ell=\sigma_{\mathrm{T}} n_{\mathrm{e} 0} \int\left[1+\left(\frac{r}{r_{\mathrm{c}}}\right)^{2}\right]^{-3 \beta / 2} \mathrm{~d} \ell \\
& \equiv n_{\mathrm{e} 0} \sigma_{\mathrm{T}} r_{\mathrm{c}} Y(\theta),
\end{aligned}
$$

where $\theta$ is the angle between the centre of cluster and the direction of observation. All the angular dependence is contained in the function

$Y(\theta)=\sqrt{\pi} \frac{\Gamma\left(\frac{3}{2} \beta-\frac{1}{2}\right)}{\Gamma\left(\frac{3}{2} \beta\right)}\left[1+\left(\frac{\theta}{\theta_{c}}\right)^{2}\right]^{\frac{1}{2}-\frac{3}{2} \beta}$,

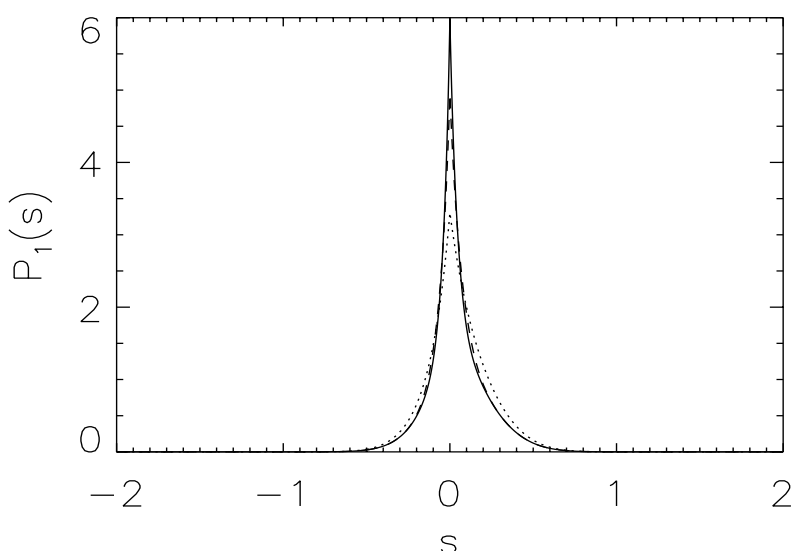

Fig. 22. The function $P_{1}(s)$ computed for a combination of two thermal populations with $k_{\mathrm{B}} T_{\mathrm{e}, 1}=8.5$ and $k_{\mathrm{B}} T_{\mathrm{e}, 2}=0.5 \mathrm{keV}$ (solid line) and with $k_{\mathrm{B}} T_{\mathrm{e}, 1}=8.5$ and $k_{\mathrm{B}} T_{\mathrm{e}, 2}=1 \mathrm{keV}$ (dashed line) is compared with that of the single thermal population with $k_{\mathrm{B}} T_{\mathrm{e}}=8.5 \mathrm{keV}$ (dotted line).

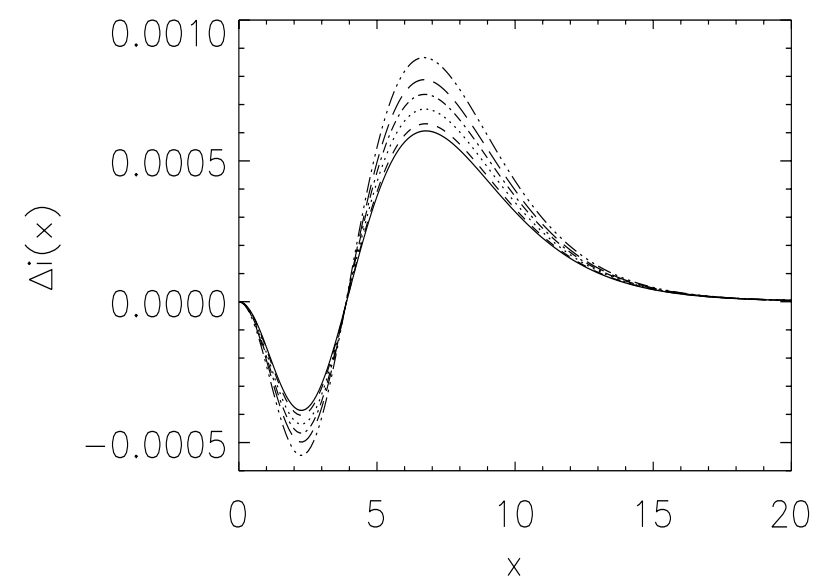

Fig. 23. The spectral distortion in units of $2\left(k_{\mathrm{B}} T_{0}\right)^{3} /(h c)^{2}$ produced by a single thermal population with $k_{\mathrm{B}} T_{\mathrm{e}, 1}=8.5 \mathrm{keV}$ and $n_{\mathrm{e}, 1}=$ $3 \times 10^{-3} \mathrm{~cm}^{-3}$ (solid line) is compared with that produced by a combination of two thermal populations with $k_{\mathrm{B}} T_{\mathrm{e}, 2}=1 \mathrm{keV}$ and $n_{\mathrm{e}, 2}=10^{-3}$ (dashed line), $3 \times 10^{-3}$ (dotted), $5 \times 10^{-3}$ (dot-dashed), $7 \times 10^{-3}$ (long dashes) and $10^{-2}$ (dot-dot-dashes) $\mathrm{cm}^{-3}$. 

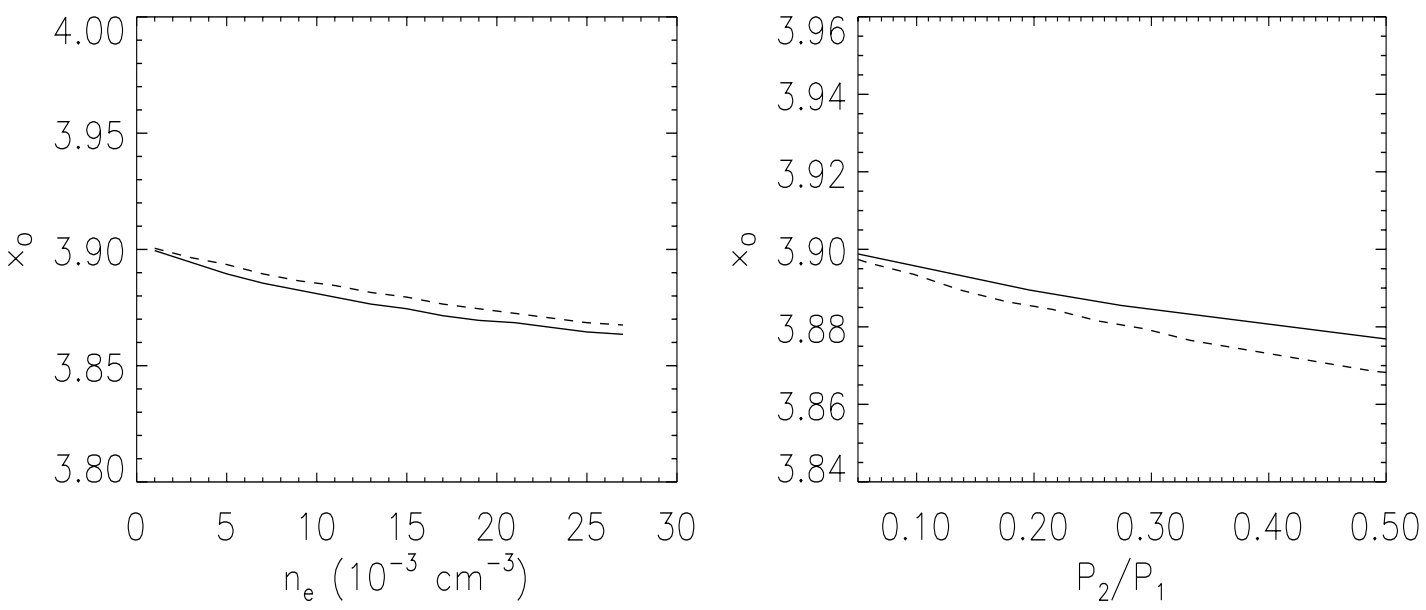

Fig. 24. The behaviour of the zero of the total $\mathrm{SZ}$ effect for a combination of two thermal populations with $k_{\mathrm{B}} T_{\mathrm{e}, 1}=8.5 \mathrm{keV}$ and $k_{\mathrm{B}} T_{\mathrm{e}, 2}=1 \mathrm{keV}$ (solid line) and $k_{\mathrm{B}} T_{\mathrm{e}, 1}=8.5 \mathrm{keV}$ and $k_{\mathrm{B}} T_{\mathrm{e}, 2}=0.5 \mathrm{keV}$ (dashed line) is shown as a function of the density $n_{\mathrm{e}, 2}$ (left panel) and of the pressure ratio $P_{2} / P_{1}$ (right panel). A value $n_{\mathrm{e}, 1}=3 \times 10^{-3} \mathrm{~cm}^{-3}$ is adopted here.
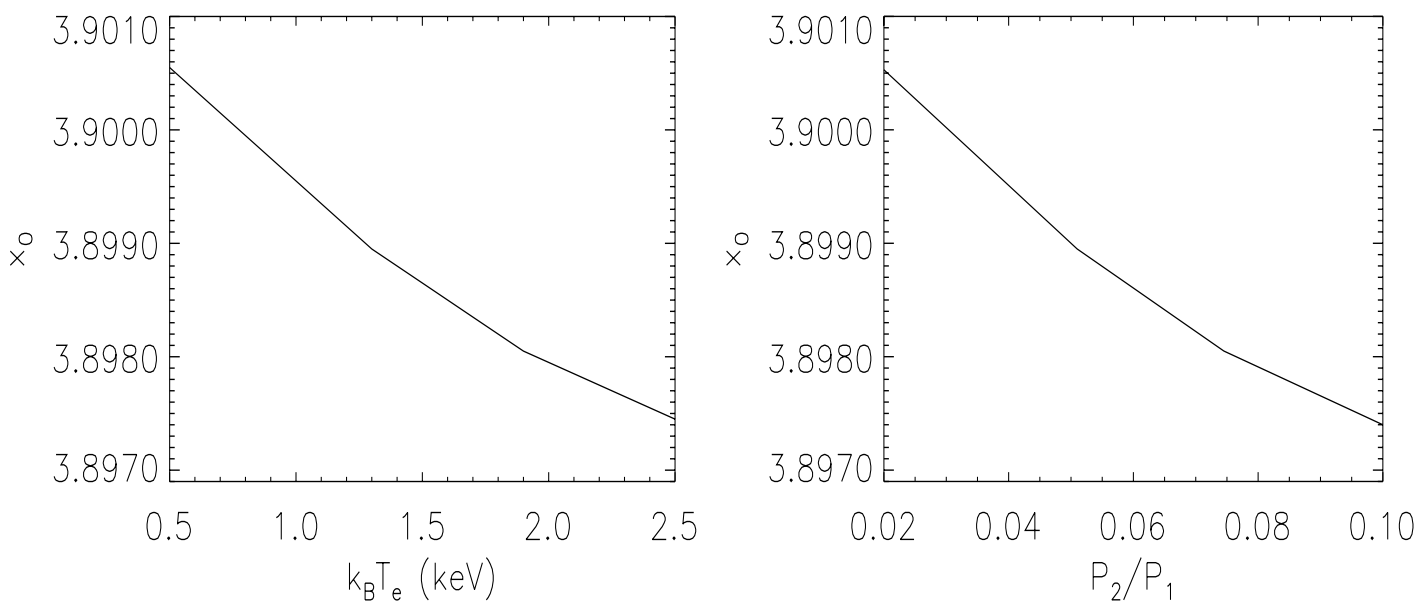

Fig. 25. The behaviour of the zero of the total $\mathrm{SZ}$ effect for a combination of two thermal populations with $k_{\mathrm{B}} T_{\mathrm{e}, 1}=8.5 \mathrm{keV}$ and $n_{\mathrm{e}, 2}=1 \times$ $10^{-3} \mathrm{~cm}^{-3}$ is shown as a function of the temperature $k_{\mathrm{B}} T_{\mathrm{e}, 2}$ (left panel) and of the pressure ratio $P_{2} / P_{1}$ (right panel). A value $n_{\mathrm{e}, 1}=3 \times 10^{-3} \mathrm{~cm}^{-3}$ is adopted here.

where $\theta_{\mathrm{c}}=r_{\mathrm{c}} / D_{\mathrm{A}}$, in terms of the angular diameter distance $D_{\mathrm{A}}$ (see Weinberg 1972).

In the following we will describe in detail, for the sake of clarity, the spatial distribution of the approximated (to first and second order in $\tau$ ) expression of the total SZ effect and we will give also the general expression for the exact calculations.

The angular dependence of the SZ-induced spectral distortion evaluated at first order in $\tau$ writes as

$\Delta i(x, \theta)=\tau(\theta)\left[j_{1}(x)-j_{0}(x)\right]$,

where $\Delta i$ is in units of $2\left(k_{\mathrm{B}} T_{0}\right)^{3} /(h c)^{2}$ and $\tau(\theta)$ is given by Eq. (70). Since the spectral distortion can be written in the general form $\Delta i(x)=y \tilde{g}(x)$, the Comptonization parameter writes as

$$
\begin{aligned}
y(\theta) & =\frac{\sigma_{\mathrm{T}}}{m_{\mathrm{e}} c^{2}} \int P \mathrm{~d} \ell=\frac{\sigma_{\mathrm{T}}}{m_{\mathrm{e}} c^{2}}\left\langle k_{\mathrm{B}} T_{\mathrm{e}}\right\rangle \int n_{\mathrm{e}}(r) \mathrm{d} \ell=\frac{\left\langle k_{\mathrm{B}} T_{\mathrm{e}}\right\rangle}{m_{\mathrm{e}} c^{2}} \tau(\theta) \\
& =\frac{\sigma_{\mathrm{T}}}{m_{\mathrm{e}} c^{2}} P_{0} r_{\mathrm{c}} Y(\theta),
\end{aligned}
$$

where the quantity $\left\langle k_{\mathrm{B}} T_{\mathrm{e}}\right\rangle$ is defined in Eq. (46) and depends only on the parameters of the electron distribution (here the central pressure is $\left.P_{0}=n_{\mathrm{e} 0}\left\langle k_{\mathrm{B}} T_{\mathrm{e}}\right\rangle\right)$. The spectral shape of the $\mathrm{SZ}$ effect at an angular distance $\theta$ from the cluster center can be written, at first order in $\tau$, as

$$
\begin{aligned}
\tilde{g}(x) & =\frac{\Delta i(x, \theta)}{y(\theta)}=\frac{\sigma_{\mathrm{T}} n_{\mathrm{e} 0} r_{\mathrm{c}} Y(\theta)}{\frac{\sigma_{\mathrm{T}}}{m_{\mathrm{e}} c^{2}} P_{0} r_{\mathrm{c}} Y(\theta)}\left[j_{1}(x)-j_{0}(x)\right] \\
& =\frac{m_{\mathrm{e}} c^{2}}{\left\langle k_{\mathrm{B}} T_{\mathrm{e}}\right\rangle}\left[j_{1}(x)-j_{0}(x)\right],
\end{aligned}
$$

and it does not contain the angular dependence of the effect. However, at second order in $\tau$ the spectral distortion writes as

$\Delta i(x, \theta)=\frac{1}{2} \tau^{2}(\theta)\left[j_{2}(x)-2 j_{1}(x)+j_{0}(x)\right]$, 
and, as a consequence, the second order approximation of the function $\tilde{g}(x)$ is

$$
\begin{aligned}
\tilde{g}(x, \theta) & =\frac{1}{2} \frac{\sigma_{\mathrm{T}}^{2} n_{\mathrm{e} o}^{2} r_{\mathrm{c}}^{2} Y^{2}(\theta)}{\frac{\sigma_{\mathrm{T}}}{m_{\mathrm{e}} c^{2}} P_{0} r_{\mathrm{c}} Y(\theta)}\left[j_{2}(x)-2 j_{1}(x)+j_{0}(x)\right] \\
& =\frac{1}{2} \frac{\sigma_{\mathrm{T}} m_{\mathrm{e}} c^{2} n_{\mathrm{e} 0}^{2} r_{\mathrm{c}} Y(\theta)}{P_{0}}\left[j_{2}(x)-2 j_{1}(x)+j_{0}(x)\right],
\end{aligned}
$$

which depends explicitly on $\theta$. It is important to notice that the angular dependence of the spectral distortion is present both in the Comptonization parameter $y$ and in the spectral shape $\tilde{g}(x)$. The dependence of the SZ effect from $\theta$ is present at all orders $n>1$ of the approximations and hence in the full, exact expression of the SZ effect. Thus, also for the study of the spatial distribution of the total SZ effect, considering the first order approximation is neither appropriate nor consistent.

For a combination of two electron populations, the total spectral distortion is given, at first order approximation in $\tau$, by the sum of the single spectral distortions of each population

$\Delta i(x, \theta)=\Delta i_{\text {th }}(x, \theta)+\Delta i_{\text {nonth }}(x, \theta)$.

This expression can be written more explicitly as

$$
\begin{aligned}
\Delta i(x, \theta)= & y_{\mathrm{th}}(\theta) \tilde{g}_{\mathrm{th}}(x)+y_{\mathrm{nonth}}(\theta) \tilde{g}_{\mathrm{nonth}}(x) \\
= & \frac{\sigma_{\mathrm{T}}}{m_{\mathrm{e}} c^{2}} P_{0, \mathrm{th}} r_{\mathrm{c}, \mathrm{X}} Y_{\mathrm{th}}(\theta) \tilde{g}_{\mathrm{th}}(x) \\
& +\frac{\sigma_{\mathrm{T}}}{m_{\mathrm{e}} c^{2}} P_{0, \text { nonth }} r_{\mathrm{c}, \mathrm{rad}} Y_{\mathrm{nonth}}(\theta) \tilde{g}_{\mathrm{nonth}}(x) \\
= & \frac{\sigma_{\mathrm{T}}}{m_{\mathrm{e}} c^{2}} P_{0, \mathrm{th}}\left[r_{\mathrm{c}, \mathrm{X}} Y_{\mathrm{th}}(\theta) \tilde{g}_{\mathrm{th}}(x)\right. \\
& \left.+r_{\mathrm{c}, \mathrm{rad}} \bar{P} Y_{\text {nonth }}(\theta) \tilde{g}_{\text {nonth }}(x)\right]
\end{aligned}
$$

where $\bar{P}=P_{0, \text { nonth }} / P_{0, \text { th }}$ is the pressure ratio at the cluster center. We assume here that the spatial distribution of the thermal and of the non-thermal electron populations are given by a $\beta$-model with core radii $r_{\mathrm{c}, \mathrm{X}}$ and $r_{\mathrm{c}, \text { rad }}$ and parameters $\beta_{\mathrm{X}}$ and $\beta_{\text {rad }}$, respectively. Such an expression is also valid in the case of the combination of two thermal populations. At higher approximation orders in $\tau$, the total spectral distortion contains cross-correlation terms of the parameters of each population and thus it is not possible to write it as a linear combination of the spectral distortions of the separate electron populations. An exact derivation (or at least the 3rd order approximation in $\tau$ ) is required to describe correctly the spatial dependence of the total SZ effect in galaxy clusters. The general calculation of the spatial distribution of the total SZ effect can be made using the exact expressions derived in Eqs. $(21-23,62,63)$ in which the spatial dependence of the thermal and non-thermal electron populations are considered.

In Fig. 26 we show the spatial behaviour of the total SZ effect in the case of a Coma-like cluster. We use specifically the following parameters: $r_{\mathrm{c}, \mathrm{X}}=0.42 h_{50}^{-1} \mathrm{Mpc}, r_{\mathrm{c}, \mathrm{rad}}=$ $0.4 h_{50}^{-1} \mathrm{Mpc}, R_{\mathrm{X}}=4.2 h_{50}^{-1} \mathrm{Mpc}, R_{\text {halo }}=1.25 h_{50}^{-1} \mathrm{Mpc}, \beta_{\mathrm{X}}=$ $0.75, \beta_{\mathrm{rad}}=0.8, n_{\mathrm{e} 0, \mathrm{X}}=2.89 \times 10^{-3} h_{50}^{2} \mathrm{~cm}^{-3}, k_{\mathrm{b}} T_{\mathrm{e}}=8.21 \mathrm{keV}$. The non-thermal electron population fitting the radio halo spectrum is taken with spectral parameters $p_{1}=1000$ and $\alpha=2.5$. The total SZ effect shows a peculiar spatial behaviour which is particularly evident at the frequencies $x \sim 2.3$ where it attains its minimum value and at frequencies $x \sim 6.5$ close to
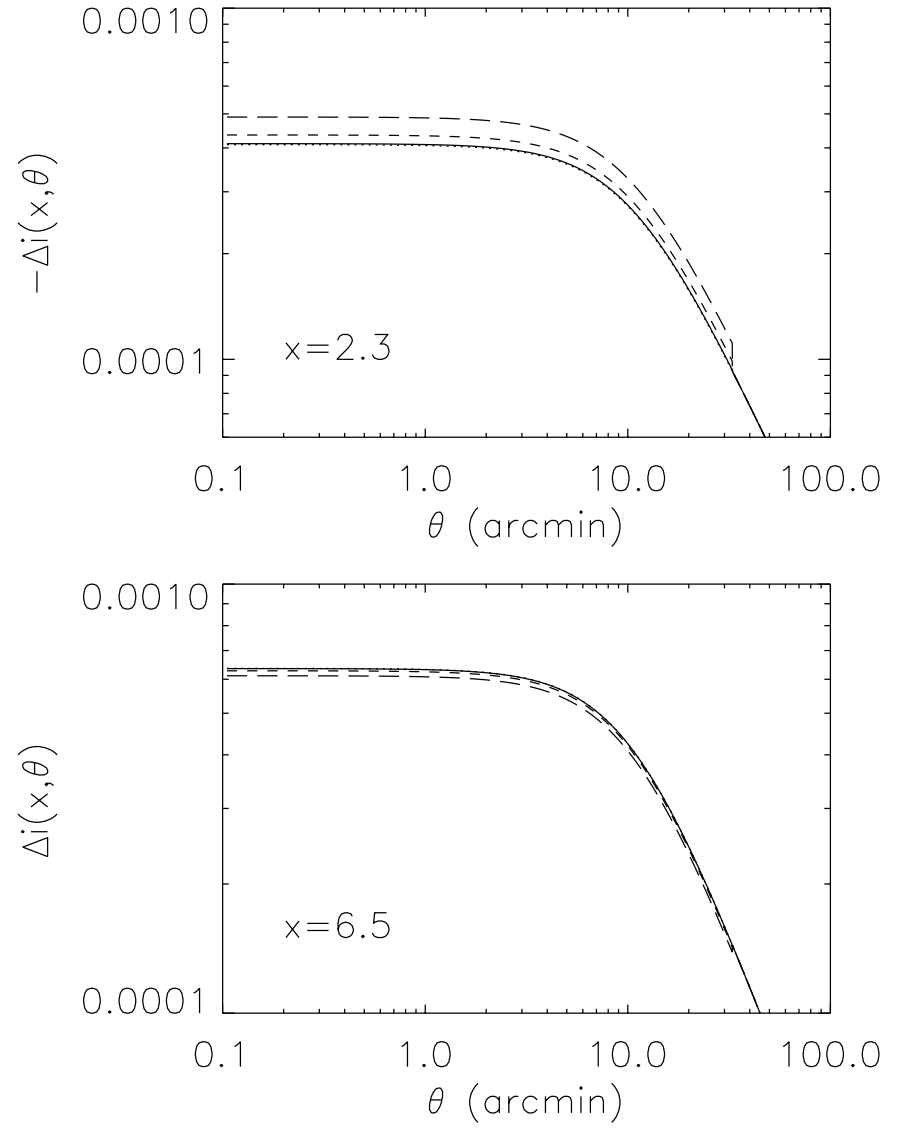

Fig. 26. The spatial dependence of the total SZ effect produced by the combination of the thermal and relativistic electrons in a Coma-like cluster. We show the thermal SZ effect (dots) and the total SZ effect with $\bar{P}=0.05$ (solid), 0.49 (short dashes) and 1.48 (long dashes). Calculations are done for an a-dimensional frequency of $x=2.3$ where the total $\mathrm{SZ}$ effect has its minimum value (upper panel) and for $x=6.5$ where the total SZ effect has its maximum value (lower panel).

its maximum. At $x \sim 2.3$ the spatial profile of the total SZ effect is predicted to be higher than that due only to the thermal population because the non-thermal SZ effect sums up in the region of the radio halo which extends up to $\sim 1.3 h_{50}^{-1} \mathrm{Mpc}$ (see Fig. 26). At larger distances from the cluster center, the SZ effect is dominated by the thermal component and resemble its spatial behaviour. An opposite behaviour is expected at frequencies higher than that of the zero of the total SZ effect. For instance at $x \sim 6.5$ the spatial profile of the total SZ effect is lower than that of the single thermal effect because the spectrum of the total SZ effect is lower than that of the thermal case. So, a depression in the spatial profile of the total $\mathrm{SZ}$ effect is expected at radii smaller than the radio halo extension $R_{\text {halo }}$. At distances larger than the radio halo extension, the total SZ effect is again dominated by the thermal component. The amplitude of the non-thermal SZ contribution depends from the pressure ratio $\bar{P}=P_{\text {rel }} / P_{\text {th }}$ as shown in Fig. 26. Such spatial features of the total SZ effect should be detectable with sensitive instruments with good spatial resolution and narrow spectral frequency band. Such instrumental capabilities can also be able to detect the transition between the ambient 
thermal SZ effect and the non-thermal SZ effect expected in radio relics and in the radio lobes of active galaxies found in the cluster environment.

\section{Application to specific galaxy clusters}

The non-thermal SZ effect may already have been observed as a part of the SZ signal from some hot galaxy clusters which possess also non thermal phenomena like radio halos or relics.

In the Coma cluster, in fact, Herbig et al. (1995) detected a strong SZ effect at the level of $y \sim 10^{-4}$ from an observation at low frequencies $v_{\mathrm{r}} \sim 30 \mathrm{MHz}$. Since the Coma cluster has a bright and extended radio halo (see, e.g., Deiss et al. 1997), it is well possible that some fraction of the SZ signal might be contributed by the non-thermal SZ effect. However, to disentangle the non-thermal SZ effect from the thermal one a detailed spectral coverage with reasonably good sensitivity in the frequency range from $x \sim 2.5$ up to $x \gtrsim 8$ is needed, with particular care to the region $x \sim 3.8-4$. At the time of the acceptance of this paper, the available SZ observations for Coma at these relevant frequencies are still lacking and we will discuss this case in more details in a further paper (Colafrancesco \& Marchegiani 2002).

In the following, nonetheless, we will discuss specifically the case of A2163 for which there are observations at different frequencies which cover the interesting part of the SZ spectrum and can be used to put constraints on the presence and on the nature of the non-thermal SZ effect in this radio-halo cluster.

\subsection{The case of $A 2163$}

The cluster A2163 $(z=0.203)$ is one of the hottest $\left(k_{\mathrm{B}} T_{\mathrm{e}} \sim\right.$ $12.5 \mathrm{keV}$ ) clusters which possesses a giant radio halo (Herbig \& Birkinshaw 1994; Feretti et al. 2001) with diameter size of $\sim(2.9 \pm 0.1) h_{50}^{-1} \mathrm{Mpc}$ which is centered on the peak of the X-ray emission. The slope of the power-law synchrotron spectrum is $\alpha_{\mathrm{r}} \approx 1.6 \pm 0.3$ and has been estimated from radio data at 1.365 and $1.465 \mathrm{GHz}$ (Feretti et al. 2001); this is consistent with the results obtained by Herbig \& Birkinshaw (1994) in the frequency range $10 \mathrm{MHz}-10 \mathrm{GHz}$. There is, however, no evidence of hard X-ray excess due to a non-thermal component in the BeppoSAX data of this cluster (Feretti et al. 2001).

A strong SZ effect has been observed in A2163 at different frequencies. In particular the SZ effect spectrum has been observed from BIMA at $28.5 \mathrm{GHz}$ (LaRoque et al. 2002), from DIABOLO at $140 \mathrm{GHz}$ (Desert et al. 1998) and from SuZIE at 140, 218 and $270 \mathrm{GHz}$ (Holzapfel et al. 1997; these data are dust-corrected in LaRoque et al. 2002) thus including and bracketing the null of the thermal SZ effect. Considering a pure thermal SZ effect, the previous data on A2163 are fitted with a Compton parameter $y_{\text {th }}=(3.65 \pm 0.40) \times 10^{-4}$ and with the addition of a kinematic SZ effect whose amplitude corresponds to a positive peculiar velocity $V_{\mathrm{p}}=415_{-765}^{+920} \mathrm{~km} \mathrm{~s}^{-1}$ (Carlstrom et al. 2002) whose large uncertainties, however, makes it consistent with a zero value. Note that, in principle, the addition of a positive-velocity kinematic SZ effect contributes to provide a deeper negative SZ signal in the region between the minimum and the zero of the SZ effect.
Here, we re-analyzed the data on A2163 trying to put constraints on the possible presence of a non-thermal SZ effect by fitting the available data with a combination of a thermal and non-thermal SZ effect.

The thermal population in A2163 has a temperature of $k_{\mathrm{B}} T_{\mathrm{e}}=12.4 \pm 0.5 \mathrm{keV}$ and a central density of $n_{\mathrm{e}, \text { th }} \simeq 6.82 \times$ $10^{-3} \mathrm{~cm}^{-3}$. The parameters describing the spatial distribution of the IC gas are $r_{\mathrm{c}}=0.36 h_{50}^{-1} \mathrm{Mpc}$ and $\beta=0.66$ (Elbaz et al. 1995; Markevitch et al. 1996). With these values the optical depth towards the cluster center is $\tau_{0, \text { th }}=1.56 \times 10^{-2}$ and the central Comptonization parameter is $y_{0, \mathrm{th}}=3.80 \times 10^{-4}$.

We show in Fig. 27 the relativistic, thermal SZ effect expected for A2163 compared to the available data. The thermal SZ effect fits the data with a $\chi^{2}=1.71$ and hence it is statistically acceptable. However, we will show in the following that the inclusion of a non-thermal component of the SZ effect is able to improve sensitively the fit to the data.

We consider a non-thermal population with a double power-law spectrum with parameters $\alpha_{1}=0.5, \alpha_{2}=2.5$, $p_{\text {cr }}=400, p_{2} \rightarrow \infty$ and we set $p_{1}$ and the density $n_{\mathrm{e}, \text { rel }}\left(\tilde{p}_{1}\right)$, with $\tilde{p}_{1}=100$, as a free parameters. We also assume that the spatial distribution of the non-thermal population is similar to that of the thermal population as indicated by the extension of the radio halo in A2163 (see, e.g., Feretti et al. 2001). In our calculations, we fix $p_{1}$ and we search for the value of $n_{\mathrm{e}, \text { rel }}\left(\tilde{p}_{1}\right)$ which minimizes the $\chi^{2}$. We show in Table 12 and in Fig. 27 the results of our calculations. We see that the data are better fitted with values of $p_{1}$ which correspond to the electrons with $p_{1} \gtrsim 10^{2}$; we see also that these electrons can produce a detectable non-thermal SZ effect with a value of the pressure $\left(P_{\text {rel }} / P_{\text {th }} \sim 0.3\right)$ and the density ratio $\bar{n} \sim 270$ which do not imply a strong influence on the dynamical and thermal state of the IC gas. A model in which $p_{1}=100$ and $n_{\mathrm{e}, \text { rel }}\left(\tilde{p}_{1}\right) \approx 2.5 \times 10^{-5} \mathrm{~cm}^{-3}$ best fits the data with a $\chi_{\min }^{2}=1.0534$, much lower than the $\chi_{\min }^{2}=1.71$ yielded by the single thermal population. The previous parameters point to a non-thermal population which carries a non negligible pressure contribution $P_{\text {rel }} \approx 0.29 P_{\text {th }}$ and which has a spectrum not extended at momenta lower than $p_{\min } \simeq 100$, corresponding to $E_{\text {min }} \approx 50 \mathrm{MeV}$.

This result does not imply that the fit with a combination of thermal plus non-thermal populations is statistically excluding the single thermal population model. However, our analysis shows that: $i$ ) a non-thermal SZ effect is produced by relativistic electrons producing radio halo emission has to be present in A2163. The $\chi^{2}$ analysis indicates that its amplitude could be appreciable (see Fig. 27) and corresponds to a pressure in relativistic particles $\left.P_{\text {rel }} \approx 0.3 P_{\text {th }} ; i i\right)$ the possibility of having SZ observations with better precision can offer the possibility to disentangle between the thermal and any non-thermal component of the SZ effect; iii) the detection of a non-thermal SZ effect can set strong constraints on the nature of the non-thermal population and on its feedback on the thermal one.

In these respects, it is appealing that the physical characteristics of the non-thermal population can be constrained through a detailed study of the SZ effect observed in the same galaxy cluster. Once the value of $p_{1}$ has been set, the quantity $n_{\mathrm{e}, \text { rel }}\left(\tilde{p}_{1}\right)$ can be constrained with a quite good precision and 
Table 12. For each value of $p_{1}$ are indicated the value of the density $n_{\mathrm{e}, \mathrm{el}}\left(\tilde{p}_{1}=100\right)$ which provides the best fit and the corresponding values of pressure $\left(\bar{P}=P_{\text {rel }} / P_{\text {th }}\right)$ and density $\left(\bar{n}=n_{\mathrm{e}, \mathrm{th}} / n_{\mathrm{e}, \text { rel }}\right)$ ratio with the corresponding value of $\chi^{2}$ for the total SZ effect in A2163 produced by a combination of a thermal population and a non-thermal double power-law population.

\begin{tabular}{ccccc}
\hline \hline$p_{1}$ & $n_{\mathrm{e}, \mathrm{rel}}\left(\tilde{p}_{1}\right)\left(\mathrm{cm}^{-3}\right)$ & $\bar{P}$ & $\bar{n}$ & $\chi^{2}$ \\
\hline 0.1 & $1.657 \times 10^{-5}$ & 0.25 & 260 & 1.0536718 \\
1 & $1.664 \times 10^{-5}$ & 0.25 & 266 & 1.0536183 \\
10 & $1.793 \times 10^{-5}$ & 0.26 & 270 & 1.0534818 \\
100 & $2.527 \times 10^{-5}$ & 0.29 & 270 & 1.0534685 \\
1000 & $7.991 \times 10^{-4}$ & 0.38 & 270 & 1.0534679 \\
10000 & $2.527 \times 10^{-2}$ & 0.46 & 270 & 1.0534679 \\
\hline
\end{tabular}

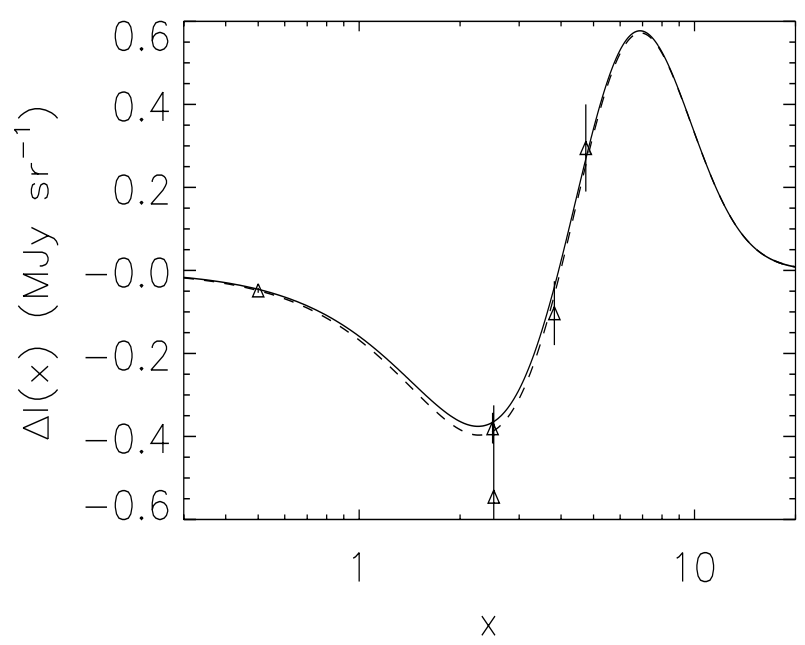

Fig. 27. Theoretical expectations for the spectrum of the SZ effect in A2163. We show the fit to the available data yielded by a thermal population (solid curve) and the expectations obtained from a combination of thermal and non-thermal populations with $p_{1}=100$ for a value of the pressure ratio $P_{\text {rel }} / P_{\text {th }}=0.29$ (dashed curve), which provides the best fit.

vice-versa. This example shows one of the potential uses of the SZ effect to obtain information on the properties of different electronic populations which are residing in the atmospheres of galaxy clusters.

Using our general approach described in the previous sections, we also considered the calculation of the total SZ effect produced by a combination of two thermal electron populations, with the warm population temperature in the range $k_{\mathrm{B}} T \sim 0.1-1 \mathrm{keV}$ and density in the range $n_{\mathrm{e}, 2} \sim 10^{-2}-$ $10^{-4} \mathrm{~cm}^{-3}$. We also considered two different spatial distributions of the hot and warm populations: in the first case the two populations have the same spatial extent and in the second one the cooler population is spatially more extended than the hotter one. In this last case we use parameters $r_{\mathrm{c}, 2}=1.5 r_{\mathrm{c}, 1}$ and $\beta_{2}=0.5$. The results of the fit to the data of A2163 are reported in Table 13 and in Fig. 28, and in Table 14 and Fig. 29. The available SZ observations allow also in this case to set constraints on the parameters $n_{\mathrm{e}, 2}$ and $T_{\mathrm{e}, 2}$ A best fit which minimizes the $\chi_{\min }^{2}$ is obtained for values $k_{\mathrm{B}} T_{\mathrm{e}, 2}=0.5-1 \mathrm{keV}$, while the case $k_{\mathrm{B}} T_{\mathrm{e}, 2}=0.1 \mathrm{keV}$ seems to be disfavoured by the data.
Table 13. For each value of $k_{\mathrm{B}} T_{\mathrm{e}, 2}$ are indicated the value of the density $n_{2}$ which provides the best fit and the corresponding values of pressure and density ratio with the corresponding value of $\chi^{2}$ for the total SZ effect in A2163 produced by a combination of a two thermal populations with the same spatial distribution.

\begin{tabular}{ccccc}
\hline \hline$k_{\mathrm{B}} T_{\mathrm{e}, 2}(\mathrm{keV})$ & $n_{\mathrm{e}, 2}\left(\mathrm{~cm}^{-3}\right)$ & $P_{2} / P_{1}$ & $n_{\mathrm{e}, 1} / n_{\mathrm{e}, 2}$ & $\chi^{2}$ \\
\hline 0.1 & $3.922 \times 10^{-2}$ & $4.64 \times 10^{-2}$ & 0.17 & 1.5633934 \\
0.5 & $8.508 \times 10^{-3}$ & $5.03 \times 10^{-2}$ & 0.80 & 1.2395049 \\
1 & $4.222 \times 10^{-3}$ & $4.99 \times 10^{-2}$ & 1.61 & 1.2307970 \\
\hline
\end{tabular}

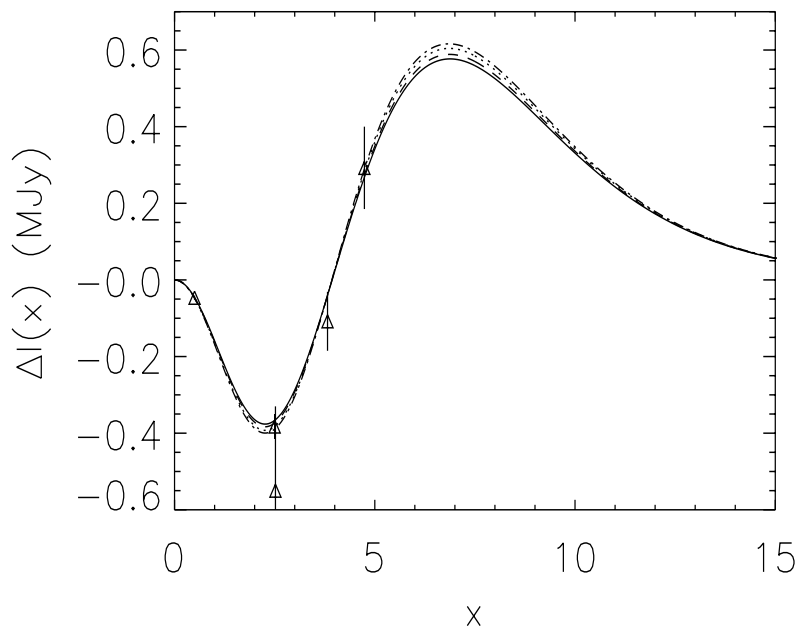

Fig. 28. The spectrum of the SZ effect in A2163 obtained from a combination of two thermal populations with $k_{\mathrm{B}} T_{\mathrm{e}, 1}=12.4 \mathrm{keV}$ and $k_{\mathrm{B}} T_{\mathrm{e}, 2}=0.5 \mathrm{keV}$ and with the same spatial distribution. We show the cases of $P_{2} / P_{1}: 0$ (solid line), $1.77 \times 10^{-2}$ (dashed line), $5.03 \times 10^{-2}$ (dotted line, which yields the best fit in this case), $5.91 \times 10^{-2}$ (dotdashed line).

Table 14. For each value of $k_{\mathrm{B}} T_{\mathrm{e}, 2}$ are indicated the value of the density $n_{2}$ which provides the best fit and the corresponding values of pressure and density ratio with the corresponding value of $\chi^{2}$ for the total SZ effect in A2163 produced by a combination of a two thermal populations with different spatial distributions.

\begin{tabular}{ccccc}
\hline \hline$k_{\mathrm{B}} T_{\mathrm{e}, 2}(\mathrm{keV})$ & $n_{\mathrm{e}, 2}\left(\mathrm{~cm}^{-3}\right)$ & $P_{2} / P_{1}$ & $n_{\mathrm{e}, 1} / n_{\mathrm{e}, 2}$ & $\chi^{2}$ \\
\hline 0.1 & $1.624 \times 10^{-2}$ & $1.92 \times 10^{-2}$ & 0.42 & 1.5911710 \\
0.5 & $3.531 \times 10^{-3}$ & $2.09 \times 10^{-2}$ & 1.93 & 1.2504161 \\
1 & $1.752 \times 10^{-3}$ & $2.07 \times 10^{-2}$ & 3.89 & 1.2412285 \\
\hline
\end{tabular}

Finally we want to remark that, in the set of models we considered in this section, the best fit to the data (with a $\chi_{\min }^{2} \approx 1.05$ ) is obtained from a combination of thermal and non-thermal populations, a case which seems to be favoured by the present data on A2163. Future SZ observations of A2163 with higher spectral resolution and sensitivity will allow to set stronger constraints on the non-thermal population. In particular, the possibility to measure accurately the frequency location of $x_{0}$ could yield direct information on the value of the pressure in relativistic particles, $P_{\text {rel }}$, confined in the cluster atmosphere and on their energy distribution. 


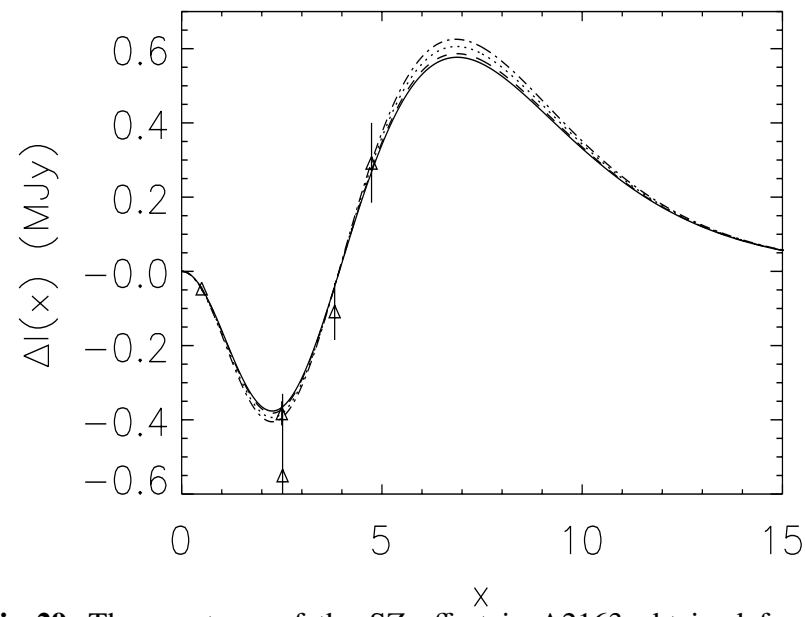

Fig. 29. The spectrum of the SZ effect in A2163 obtained from a combination of two thermal populations with $k_{\mathrm{B}} T_{\mathrm{e}, 1}=12.4 \mathrm{keV}$ and $k_{\mathrm{B}} T_{\mathrm{e}, 2}=0.5 \mathrm{keV}$ and with different spatial distributions as explained in the text. We show the cases of $P_{2} / P_{1}: 0$ (solid line), $5.91 \times 10^{-3}$ (dashed line), $2.09 \times 10^{-2}$ (dotted line, which yields the best fit), $2.96 \times 10^{-2}$ (dot-dashed line).

\section{Discussion and conclusions}

Since many galaxy clusters with available SZ measurements show also the presence of an extended radio halo and other nonthermal phenomena, it is relevant to understand quantitatively the relevance of the non-thermal SZ effect in galaxy clusters and to disentangle it from the true thermal SZ effect which is extensively used for cosmological studies. It is also relevant to use the limits on the non-thermal SZ effect to put constraints on the spectrum of the non-thermal population which is responsible for the high-energy phenomena occurring in galaxy clusters. This proves to be a powerful and complementary analysis of the specific studies of non-thermal phenomena carried out at other wavelenghts.

In such a context, we have shown in this paper that the spectra of the thermal and non-thermal SZ effects are distinctly different and that the overall spectrum of the SZ effects measures the energy densities in the thermal and in the non-thermal electron populations, separately. We have calculated here the spectral shape of the non-thermal SZ effect in the Thomson limit using an exact approach which makes use of the full relativistic formalism and goes beyond the Kompaneets and the single scattering approximation. Moreover we evaluated, for the first time in a consistent way, the total SZ effect arising from a combination of different electron populations residing in the same galaxy cluster: we considered specifically the cases of $i$ ) a thermal plus a non-thermal electron populations and ii) two thermal populations with different temperatures and densities. Such a derivation can be obtained, nonetheless, for an arbitrary set of different electron populations.

We have shown in our approach that the spectral distortion of the CMB due to both the thermal and the non-thermal $\mathrm{SZ}$ effect can be written in a general, single form which depends separately on the Comptonization parameter and on the spectral shape of the specific SZ effect. Such an expression is valid for both thermal and non-thermal SZ effects produced by single electron populations as well as for the overall SZ effect produced by a combination of different electron populations. We have also shown that the spectral dependence of the SZ effect, contained in the function $\tilde{g}(x)$, cannot be separated from the dependence on the cluster parameters even at the first order approximation in $\tau$, where $\tilde{g}(x)$ depends on the cluster (effective) temperature. At higher-order approximation in $\tau, \tilde{g}(x)$ depends, moreover, also on $\tau$. In the exact approach, the spectral shape of the SZ effect depends strongly on the cluster parameters themselves and specifically from the pressure and the optical depth of the considered electron population.

We have calculated in detail the precision at which each approximation order in $\tau$ reproduces the exact form of the SZ effect: we have shown that the third order approximation in $\tau$ yields a precision $\lesssim 1 \%$ at each interesting frequency for both the non-thermal and the thermal SZ effect.

We have shown that the main spectral differences for the non-thermal SZ effect with respect to the thermal SZ effect are a maximum of the spectral shape which is moved to higher and higher frequencies for increasing values of the lower cutoff in the momentum distribution, $p_{1}$. As a consequence, also the null of the non-thermal SZ effect, $x_{0}$, is moved to higher frequencies for increasing values of $p_{1}$. Since the pressure $P_{\text {rel }}$ of the non-thermal electron distribution depends on $p_{1}$, the position of $x_{0}$ decreases with increasing values of $P_{\text {rel }}$ for a fixed normalization of the non-thermal electron density.

We have shown in this paper that the shape of the exact nonthermal SZ effect is different from the 1st order approximation estimate used so far by different authors (Birkinshaw 1999; Ensslin \& Kaiser 2000; Blasi et al. 2000; Shimon \& Rephaeli 2002). We have shown that the 3rd order approximation is required to reproduce the exact results within a $\sim 1 \%$ precision at all relevant values of $x$.

We have also shown that the SZ effect from a single nonthermal population in clusters with non-thermal phenomena is not a realistic description of the effect. So, we have derived for the first time a consistent expression for the total SZ effect produced by a combination of a thermal and a non-thermal populations, like is the realistic case in many galaxy clusters. The shape and the amplitude of the total SZ effect is quite different from that suggested in previous papers (Ensslin \& Kaiser 2000; Blasi et al. 2000). The main difference in the derivation of the overall SZ effect is that it is not just given by the sum of the separate thermal and non-thermal contributions (as is the case for the first order approximation in $\tau$ ) but it is given by a properly weighted (according to the optical depths of each separate population) combination of the thermal and non-thermal contributions, as discussed in Sect. 4. Also, we considered in our approach the effect of multiple scattering and higher $(n>1)$ order approximation terms in the analytical derivation of the overall SZ effect. Moreover, we also discussed not only the case of $i$ ) a single power-law electron spectrum (as was done also by the previous authors) but also the more consistent case of a $i$ i) double power-law electron spectrum which is able to fit both the radio halo and the hard X-ray spectra in clusters. We showed that, while in the case $i$ ) low-energy electrons are the dominant source of non-thermal Compton scattering and produce an increase of SZ signal in the region $x \gtrsim 7$ (where the thermal SZ effect has its maximum), in the case 
ii) high-energy electrons are the dominant source of nonthermal SZ effect and produce very large shifts of CMB photon frequencies producing a deprivation of photons also in the region of the maximum of the thermal SZ effect $(x \sim 7)$.

A relevant result of our analysis shows that the location of the zero of the total SZ effect depends on the pressure ratio $\bar{P}=P_{\text {rel }} / P_{\text {th }}$ between the relativistic and thermal pressures of the two different electron populations. It increases non-linearly with $\bar{P}$ up to values $x_{0} \sim 4.2$ for $\bar{P}$ up to values $\approx 1.5$. This is a unique and relevant feature of the non-thermal SZ effect in clusters since it yields, in principle, a direct measure of the total pressure in relativistic non-thermal particles in the cluster atmosphere, an information which is not easily accessible from the study of other non-thermal phenomena like radio halos and/or EUV or hard X-ray emission excesses. Thus, the detailed observations of the frequency shift of $x_{0}$ in galaxy clusters provides unambiguously a constraint to the relativistic particle content in the cluster atmosphere. Also, such a measurement is crucial to determine the true amount of kinematic SZ effect in clusters since it is usually estimated from the residual SZ signal at the location of the zero of the thermal (relativistic) SZ effect. Due to the steepness of the SZ spectral shape in the region of the null of the overall SZ effect, the possible additional non-thermal SZ signal must be determined precisely in order to derive reliable limits for the kinematic SZ effect. This fact can severely limit the possibility to measure effectively the kinematic SZ effect in radio-halo clusters. An accurate subtraction of the non-thermal SZ effect needs a specific observational strategy with measurements in at least three frequency ranges $x \sim 2-3,3.8-4.5$ and $\sim 6-8$.

We verified that our approach is consistent with the exact derivation of the monochromatic redistribution function containing both the relativistic and quantum corrections already given by Fargion et al. (1997) and Sazonov \& Sunyaev (2000) in the Thomson limit and in the single scattering limit. In the Thomson limit, nonetheless, we have generalized the full derivation of the $\mathrm{SZ}$ effect to the case of multiple scattering and to any approximation order in $\tau$.

After the submission of our paper, Shimon \& Rephaeli (2002) presented a computation of the SZ effect produced by non-thermal electron populations in a few clusters like Coma and A2199. However, we want to stress that their derivation of the non-thermal SZ effect is still approximate since they consider only the first order approximation in $\tau$ (in which case the total SZ effect is the sum of the thermal and non-thermal effects) and the single scattering limit. We have shown in our paper that such an approximated description is neither complete nor precise since at least the third order approximation calculation in $\tau$ is required to reproduce the exact derivation of the total SZ effect within \% accuracy level.

We have also generalized our derivation of the total SZ effect in galaxy clusters to the case of a combination of different thermal electron populations (see Sect. 5). Any additional cool IC gas component produces a tightening of the photon redistribution function, an increase in the total optical depth and hence a substantial change in the spectral distortion at the minimum and at the maximum of the SZ effect. The location of the zero of the SZ effect, $x_{0}$ also decreases in frequency due to the presence of the cooler component which decreases the pressure ratio $P_{2} / P_{1}$. Thus, the possible detection of an additional cold component in the cluster atmosphere through observations of the total (thermal plus thermal) SZ effect, allow to test the possible thermal origin of the EUV excess observed in several nearby clusters (Lieu et al. 1999, 2000). Our analysis of the cluster A2163 does not indicate a relevant role of the warmer component to the total pressure of the cluster atmosphere, consistently with the present indication of the absence of a strong EUV excess in this cluster. We will address the specific analysis of other clusters elsewhere.

Beyond the relevance of the study of the non-thermal SZ effect as a bias for the cosmologically relevant thermal SZ effect, the non-thermal SZ effect has also a crucial astrophysical relevance as a barometer for the presence of any additional population of electrons with both a non-thermal or a thermal energy spectrum. In fact, the non-thermal SZ effect actually measures the total pressure of the non-thermal electron population and hence yields constraints to its energy spectrum. Analogously, the additional thermal SZ effect produced by a cooler thermal component provides information on its temperature and density. Specifically, we have shown that combining information from the cluster radio-halo observation and non-thermal SZ measurements, one is able to determine the shape and the extension of the relativistic electron spectrum. Using the available observation of one of the best example of radio-halo clusters with multifrequency observations of the SZ effect, A2163, we applied our method and derived a limit on the low-energy cutoff of the relativistic electron spectrum of $E_{\min } \sim 50 \mathrm{MeV}$, as well as constraints on the momentum spectrum which is required to be flat enough $\left(f_{\mathrm{e}} \sim p^{-0.5}\right)$ below $p \sim 400$ to avoid destructive feedback effects on the thermal IC gas.

Relativistic electrons with such flat energy spectrum do not produce a relevant extra heating and/or extra X-ray emission with respect to the thermal IC gas in A2163. Also the IC energy losses of such relativistic electrons do not yield a substantial hard X-ray emission, in agreement with the available limit on A2163 obtained from BeppoSAX observations.

We have finally shown that the presence of a non-thermal SZ effect also influence the spatial profile of the total SZ effect of a typical radio-halo cluster. In fact, the region occupied by the radio halo shows an increment (decrement) of the signal at frequencies near the minimum (maximum) of the SZ effect. This happens as a consequence of the difference in the spectral shapes of the non-thermal SZ effect with respect to the thermal one.

The specific spectral and spatial features of the non-thermal SZ effect makes it possible to detect it through a multifrequency observation with high sensitivity and narrow-band detectors. The optimal observational strategy is to observe galaxy clusters in the frequency range $x \sim 2-8$ where the peculiar spectral features allow clearly to disentangle the non-thermal SZ effect from the thermal one. The PLANCK surveyor experiment has the capabilities to detect and map the non-thermal SZ effect in a large number of nearby radio-halo clusters. However, dedicated experiment with high sensitivity and narrow band spectral coverage are also adequate to detect the non-thermal SZ effect in radio-halo galaxy clusters. 
Acknowledgements. We thank the referee, N. Itoh, for several useful comments and suggestions which allow us to improve the presentation of our results.

\section{Appendix A: The total SZ effect for a combination of two electron populations: analytic approximations}

In this appendix, we derive detailed expressions for $I_{\text {tot }}(x)$ which are approximated at first and second order in $\tau$ according to the general expression:

$I(x)=I_{0}(x)+\tau\left[J_{1}(x)-J_{0}(x)\right]+\frac{1}{2} \tau^{2}\left[J_{2}(x)-2 J_{1}(x)+J_{0}(x)\right]$.

We remind the reader that the function $J_{1}(x)$ is given by Eq. (18):

$J_{1}(x)=\int_{-\infty}^{+\infty} I_{0}\left(x \mathrm{e}^{-s}\right) P_{1}(s) \mathrm{d} s$

where $P_{1}(s)$ is given by Eq. (5):

$P_{1}(s)=\int_{0}^{\infty} \mathrm{d} p f_{\mathrm{e}}(p) P_{\mathrm{s}}(s ; p)$.

Inserting Eq. (52) in the previous equation for $P_{1}(s)$, we obtain:

$$
\begin{aligned}
P_{1}(s) & =c_{\mathrm{A}} \int_{0}^{\infty} \mathrm{d} p f_{\mathrm{A}}(p) P_{\mathrm{s}}(s ; p)+c_{\mathrm{B}} \int_{0}^{\infty} \mathrm{d} p f_{\mathrm{B}}(p) P_{\mathrm{s}}(s ; p) \\
& \equiv c_{\mathrm{A}} P_{1 \mathrm{~A}}(s)+c_{\mathrm{B}} P_{1 \mathrm{~B}}(s) .
\end{aligned}
$$

Inserting Eq. (A.4) in Eq. (A.2), we also obtain the expression:

$$
\begin{aligned}
J_{1}(x) & =c_{\mathrm{A}} \int_{-\infty}^{+\infty} I_{0}\left(x \mathrm{e}^{-s}\right) P_{1 \mathrm{~A}}(s) \mathrm{d} s+c_{\mathrm{B}} \int_{-\infty}^{+\infty} I_{0}\left(x \mathrm{e}^{-s}\right) P_{1 \mathrm{~B}}(s) \mathrm{d} s \\
& \equiv c_{\mathrm{A}} J_{1 \mathrm{~A}}(x)+c_{\mathrm{B}} J_{1 \mathrm{~B}}(x) .
\end{aligned}
$$

Inserting Eqs. (A.5), (55) and (54) in Eq. (A.1), we derive, at first order in $\tau$, the expression:

$$
\begin{aligned}
\Delta I_{\mathrm{tot}}^{(1)}(x) & =\left(\tau_{\mathrm{A}}+\tau_{\mathrm{B}}\right)\left[c_{\mathrm{A}} J_{1 \mathrm{~A}}(x)+c_{\mathrm{B}} J_{1 \mathrm{~B}}(x)-J_{0}(x)\right] \\
& =\tau_{\mathrm{A}} J_{1 \mathrm{~A}}(x)+\tau_{\mathrm{B}} J_{1 \mathrm{~B}}(x)-\tau_{\mathrm{A}} J_{0}(x)-\tau_{\mathrm{B}} J_{0}(x) \\
& =\tau_{\mathrm{A}}\left[J_{1 \mathrm{~A}}(x)-J_{0}(x)\right]+\tau_{\mathrm{B}}\left[J_{1 \mathrm{~B}}(x)-J_{0}(x)\right] .
\end{aligned}
$$

As expected, the SZ effect at first order in $\tau$ is given by the sum of the separate SZ effects produced by the two electron distributions, respectively.

To evaluate the effect at second order in $\tau$, it is necessary to calculate the expression:

$J_{2}(x)=\int_{-\infty}^{+\infty} I_{0}\left(x \mathrm{e}^{-s}\right) P_{2}(s) \mathrm{d} s$.

The function $P_{2}(s)$ can be derived by Eq. (6) using Eq. (A.4):

$$
\begin{aligned}
P_{2}(s)= & P_{1}(s) \otimes P_{1}(s) \\
= & {\left[c_{\mathrm{A}} P_{1 \mathrm{~A}}(s)+c_{\mathrm{B}} P_{1 \mathrm{~B}}(s)\right] \otimes\left[c_{\mathrm{A}} P_{1 \mathrm{~A}}(s)+c_{\mathrm{B}} P_{1 \mathrm{~B}}(s)\right] } \\
= & c_{\mathrm{A}}^{2} P_{1 \mathrm{~A}}(s) \otimes P_{1 \mathrm{~A}}(s)+2 c_{\mathrm{A}} c_{\mathrm{B}} P_{1 \mathrm{~A}}(s) \otimes P_{1 \mathrm{~B}}(s) \\
& +c_{\mathrm{B}}^{2} P_{1 \mathrm{~B}}(s) \otimes P_{1 \mathrm{~B}}(s) \\
\equiv & c_{\mathrm{A}}^{2} P_{2 \mathrm{AA}}(s)+2 c_{\mathrm{A}} c_{\mathrm{B}} P_{2 \mathrm{AB}}(s)+c_{\mathrm{B}}^{2} P_{2 \mathrm{BB}}(s) .
\end{aligned}
$$

Inserting this expression in Eq. (A.7), we obtain:

$$
\begin{aligned}
J_{2}(x)= & c_{\mathrm{A}}^{2} \int_{-\infty}^{+\infty} I_{0}\left(x \mathrm{e}^{-s}\right) P_{2 \mathrm{AA}}(s) \mathrm{d} s \\
& +2 c_{\mathrm{A}} c_{\mathrm{B}} \int_{-\infty}^{+\infty} I_{0}\left(x \mathrm{e}^{-s}\right) P_{2 \mathrm{AB}}(s) \mathrm{d} s \\
& +c_{\mathrm{B}}^{2} \int_{-\infty}^{+\infty} I_{0}\left(x \mathrm{e}^{-s}\right) P_{2 \mathrm{BB}}(s) \mathrm{d} s \\
\equiv & c_{\mathrm{A}}^{2} J_{2 \mathrm{AA}}(x)+2 c_{\mathrm{A}} c_{\mathrm{B}} J_{2 \mathrm{AB}}(x)+c_{\mathrm{B}}^{2} J_{2 \mathrm{BB}}(x) .
\end{aligned}
$$

From Eq. (A.1) we derive the expression of the second order correction to the total distorted spectrum

$$
\begin{aligned}
\Delta I_{\text {tot }}^{(2)}(x)= & \frac{1}{2}\left(\tau_{\mathrm{A}}+\tau_{\mathrm{B}}\right)^{2}\left[c_{\mathrm{A}}^{2} J_{2 \mathrm{AA}}+2 c_{\mathrm{A}} c_{\mathrm{B}} J_{2 \mathrm{AB}}\right. \\
& \left.+c_{\mathrm{B}}^{2} J_{2 \mathrm{BB}}-2 c_{\mathrm{A}} J_{1 \mathrm{~A}}-2 c_{\mathrm{B}} J_{1 \mathrm{~B}}+J_{0}\right] \\
= & \frac{1}{2}\left\{\tau_{\mathrm{A}}^{2} J_{2 \mathrm{AA}}+2 \tau_{\mathrm{A}} \tau_{\mathrm{B}} J_{2 \mathrm{AB}}-2 \tau_{\mathrm{A}}\left(\tau_{\mathrm{A}}+\tau_{\mathrm{B}}\right) J_{1 \mathrm{~A}}\right. \\
& \left.-2 \tau_{\mathrm{B}}\left(\tau_{\mathrm{A}}+\tau_{\mathrm{B}}\right) J_{1 \mathrm{~B}}+\left(\tau_{\mathrm{A}}^{2}+2 \tau_{\mathrm{A}} \tau_{\mathrm{B}}+\tau_{\mathrm{B}}^{2}\right) J_{0}\right\} \\
= & \frac{1}{2}\left\{\tau_{\mathrm{A}}^{2}\left[J_{2 \mathrm{AA}}-2 J_{1 \mathrm{~A}}+J_{0}\right]+\tau_{\mathrm{B}}^{2}\left[J_{2 \mathrm{BB}}-2 J_{1 \mathrm{~B}}+J_{0}\right]\right. \\
& \left.+2 \tau_{\mathrm{A}} \tau_{\mathrm{B}}\left[J_{2 \mathrm{AB}}-J_{1 \mathrm{~A}}-J_{1 \mathrm{~B}}+J_{0}\right]\right\} .
\end{aligned}
$$

Notice that at second order in $\tau$ there is an additional term describing the probability that a CMB photon can suffer first a scattering from the electrons of the distribution $f_{\mathrm{A}}$ and then another scattering from the electrons of the distribution $f_{\mathrm{B}}$.

In a similar approach, we can derive the further corrections to the total distorted spectrum evaluated at any order in $\tau$; these will contain a larger and larger number of cross-scattering terms. In a completely analogous way, we can also consider the case of three and more electron distributions.

\section{References}

Abramowitz, M., \& Stegun, I. A. Handbook of Mathematical Functions with Formulas, Graphs and Mathematical Tables (Dover Book, N.Y.)

Arnaud, K. A., et al. 1994, ApJ, 436, L67

Birkinshaw, M. 1999, Phys. Rep., 310, 97

Blasi, P., \& Colafrancesco, S. 1999, Astropart. Phys., 122, 169

Blasi, P., Stebbins, A., \& Olinto, A. 2000, ApJ, 535, L71

Bowyer, S. 2000, AAS, 32, 1707

Carlstrom, J. E., Holder, G. P., \& Reese, E. D. 2002, ARA\&A, 40, 643

Cavaliere, A., \& Fusco-Femiano, R. 1976, A\&A, 49, 137

Challinor, A., \& Lasenby, A. 1998, ApJ, 499, 1

Colafrancesco, S. 2001, in Constructing the Universe with Clusters of Galaxies, ed. D. Gerbal, \& F. Durret

Colafrancesco, S. 2002, in Frontier of CMB Physics, ed. J. Bartlett, et al., in press

Colafrancesco, S., \& Mele, B. 2001, ApJ, 562, 24

Colafrancesco, S., \& Marchegiani, P. 2002, A\&A, submitted

David, L. P., et al. 1993, ApJ, 412, 479

Deiss, B., et al. 1997, A\&A, 321, 55

Desert, F., et al. 1998, New Astron., 3, 655

Dogiel, V. A. 2000, A\&A, 357, 66

Dolgov, A. D., et al. 2001, ApJ, 554, 74 [preprint astro-ph/0010412]

Ensslin, T., \& Kaiser, C. 2000, A\&A, 360, 417

Fargion, D., Konoplich, R. V., \& Salis, A. 1997, J. Phys. C, 74, 571 
Fargion, D., \& Salis, A. 1998, Phys. Uspekhi, 41, 823

Feretti, L. 2001, in Constructing the Universe with Clusters of Galaxies, ed. D. Gerbal, \& F. Durret

Feretti, L., et al. 2001, A\&A, 373, 106

Fusco-Femiano, R., et al. 1999, ApJ, 513, L21

Fusco-Femiano, R., et al. 2000, ApJ, 534, L7

Giovannini, G., et al. 1993, ApJ, 406, 399

Henriksen, M. 1999, ApJ, 511, 666

Herbig, T., \& Birkinshaw, M. 1994, BAAS, 26, No. 4, 1403

Herbig, T., et al. 1995, ApJ, 449, L5

Holzapfel, W. L., et al. 1997, ApJ, 481, 35

Itoh, N., Kohyama, Y., \& Nozawa, S. 1998, ApJ, 502, 7

Itoh, N., et al. 2001, MNRAS, 327, 567

Itoh, N., et al. 2002, A\&A, 382, 722

Kaastra, J., et al. 1999, ApJ, 519, L119

Kaastra, J., et al. 2002, ApJ, 574, L1

Kompaneets, A. S. 1957, Soviet Phys. JETP, 4, 730

LaRoque S.J., et al. 2002, ApJ, submitted [preprint astro-ph/0204134]

Lieu, R., et al. 1999, ApJ, 510, L25

Lieu, R., et al. 2000, A\&A, 364, 497
Markevitch, M., et al. 1994, ApJ, 436, L71

McKinnon, M. M., et al. 1991, AJ, 101, 2026

Mushotzky, R. F., \& Scharf, C. A. 1997, ApJ, 482, L13

Nozawa, S., Itoh, N., Kawana, Y., \& Kohyama, Y. 2000, ApJ, 536, 31

Petrosian, V. 2001, ApJ, 557, 560

Rephaeli, Y., Gruber, W., \& Blanco, P. 1999, ApJ, 511, L21

Sarazin, C. L. 1988, X-ray emission from clusters of galaxies (Cambridge University Press)

Sarazin, C. L. 1999, ApJ, 520, 529

Sarazin, C. L., \& Kempner, J. C. 2000, ApJ, 533, 73

Sazonov, S.Y. \& Sunyaev, R.A. 2000, ApJ, 543, 28

Shimon, M., \& Rephaeli, Y. 2002, [preprint astro-ph/0204429]

Stebbins, A. 1997, in The Cosmic Microwave background (Kluwer Acad. Press,), p. 241, [preprint astro-ph/9705178]

Sunyaev, R. A., \& Zel'dovich, Ya. B. 1972, Comments Astrophys. Space Sci., 4, 173

Sunyaev, R. A., \& Zel'dovich, Ya. B. 1980, ARA\&A, 18, 537

Taylor, G. B., \& Wright, E. L. 1989, ApJ, 339, 619

Tucker, W., et al. 1998, ApJ, 496, L5

Weinberg, S. 1972, Gravitation and Cosmology (Academic Press)

Zel'dovich, Ya. B., \& Sunyaev, R. A. 1969, AP\&SS, 4, 173 